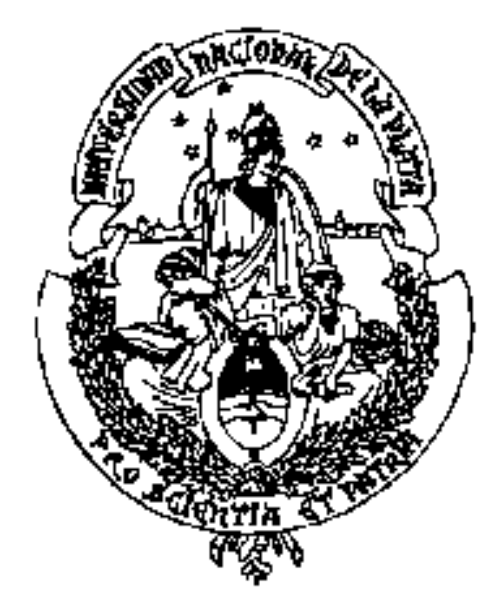

\title{
Vulnerabilidad a la pobreza: Aplicaciones para América Latina
}

\author{
Mauricio Eugenio Gallardo Altamirano
}

Tesis Doctoral

Doctorado en Economía

Facultad de Ciencias Económicas

Universidad Nacional de La Plata

Director de Tesis:

Dr. Luciano Di Gresia

La Plata, 12 de diciembre del 2013 
Vulnerabilidad a la pobreza:

Aplicaciones para América Latina.

Tesis Doctoral.

Mauricio Gallardo Altamirano, 2013.

@Derechos reservados del autor para los capítulos 1 y 3 .

@Derechos concedidos por el autor a la revista Economics Letters de la editorial ELSEVIER, para el contenido principal del capítulo 2.

Manuscrito original con registro de propiedad intelectual. 


\section{Índice general}

$\begin{array}{ll}\text { Introducción } & 9\end{array}$

1. El problema de la identificación $\quad 13$

1.1. Introducción . . . . . . . . . . . . . . . . . . . . . . 13

1.2. Vulnerabilidad como exposición al riesgo . . . . . . . . . . . . . 15

1.2.1. Vulnerabilidad como exposición al riesgo de los hogares de bajos ingresos . . . . . . . . . . . . . . . . 15

1.2.2. Enfoque de la línea de pobreza extendida . . . . . . . . . . . . 20

1.2.3. La Propuesta de Béné . . . . . . . . . . . . . . . . . . 23

1.2.4. Vulnerabilidad como percepción subjetiva del riesgo a la baja . . . 25

1.2.5. Enfoque de Dutta-Foster-Mishra . . . . . . . . . . . . . . . . 29

1.3. Enfoques de vulnerabilidad como pobreza esperada . . . . . . . . . . . 36

1.3.1. Vulnerabilidad como probabilidad de pobreza futura . . . . . . . . 36

1.3.2. Vulnerabilidad como baja utilidad esperada . . . . . . . . . . . . . 42

1.3.3. Enfoque de Calvo y Dercon . . . . . . . . . . . . . . . . . . . 46

1.3.4. La Propuesta de Chiwaula-Witt-Waibel . . . . . . . . . . . . . . . . 48

1.4. En busca de una síntesis conceptual . . . . . . . . . . . . . . . . . 51

1.4.1. Vulnerabilidad y vulnerabilidad a la pobreza . . . . . . . . . 51

1.4.2. Elementos claves de la vulnerabilidad a la pobreza . . . . . . . . . 53

1.5. Conclusiones . . . . . . . . . . . . . . . . . 56

$\begin{array}{ll}\text { 2. El problema de la medición } & 63\end{array}$

2.1. Introducción . . . . . . . . . . . . . . . . . . . . 63

2.2. Marco Conceptual . . . . . . . . . . . . . . . . . . . . 64

2.2.1. Dos tipos de vulnerabilidad a la pobreza . . . . . . . . . . . . 64 
2.2.2. Formalización del enfoque . . . . . . . . . . . . . 66

2.3. Un índice de vulnerabilidad de la población . . . . . . . . . . . . 67

2.4. Una ilustración empírica para el Perú . . . . . . . . . . . . . . . . . 71

2.4.1. Motivación del ejemplo y datos utilizados . . . . . . . . . . . . . . . 71

2.4.2. Estrategia de estimación empírica . . . . . . . . . . . . . . . 72

2.4.3. Principales Resultados . . . . . . . . . . . . . . . . . . . . 75

2.4.4. Ejercicios de robustez: comparación con los resultados de otros in-

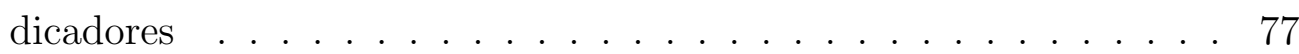

2.5. Conclusiones . . . . . . . . . . . . . . . . . . . . 78

3. Evidencia empírica para América Latina 83

3.1. Introducción . . . . . . . . . . . . . . . . . . . . . 83

3.2. Concepto y medida de vulnerabilidad . . . . . . . . . . . 86

3.3. Estrategia de estimación empírica . . . . . . . . . . . . . . . . . 89

3.4. Descripción de los datos utilizados . . . . . . . . . . . . . . . . . 93

3.5. Discusión de resultados . . . . . . . . . . . . . . . . . . . 96

3.5.1. Argentina . . . . . . . . . . . . . . . . . 96

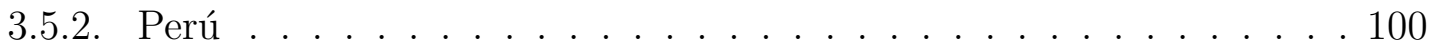

3.5.3. Nicaragua . . . . . . . . . . . . . . . . . . . . 103

3.5.4. Chile . . . . . . . . . . . . . . . . . . 105

3.5.5. Contrastes de las hipótesis planteadas . . . . . . . . . . . . 108

3.5.6. Pruebas de robustez . . . . . . . . . . . . . . . . 111

3.6. Conclusiones . . . . . . . . . . . . . . . . . . . 115

$\begin{array}{lr}\text { Conclusiones } & 125\end{array}$

$\begin{array}{ll}\text { Contribución de la tesis a la literatura } & 129\end{array}$ 


\section{Índice de tablas}

2.1. Medidas FGT de Pobreza y Vulnerabilidad, Perú (2007-2010). . . . . . . . 76

2.2. Medidas alternativas de Vulnerabilidad para diferentes parámetros de riesgo, Perú (2007-2010) . . . . . . . . . . . . . . . . . . 78

3.1. Estadísticas descriptivas del ingreso per cápita de los hogares, normalizado por la línea de pobreza. . . . . . . . . . . . . . . . . . . 96

3.2. Medidas FGT de Pobreza y Vulnerabilidad, Argentina (2003-2011). . . . . 98

3.3. Medidas FGT de Pobreza y Vulnerabilidad, Perú (2004-2010). . . . . . . . 101

3.4. Medidas FGT de Pobreza y Vulnerabilidad, Nicaragua (1998-2005). . . . . 105

3.5. Medidas FGT de Pobreza y Vulnerabilidad, Chile (1996-2006). . . . . . . . 108

3.6. Contraste ANOVA sobre la importancia de la vulnerabilidad severa. . . . . 110

3.7. Contraste Kruskal Wallis sobre la importancia de la vulnerabilidad severa. 111

3.8. Regresión simple entre la participación de la vulnerabilidad severa en la tasa de incidencia de la vulnerabilidad total (share) y el logaritmo de la tasa de incidencia de la pobreza (1_pobreza). . . . . . . . . . . . . . . . . . 112

3.9. Indicadores alternativos de vulnerabilidad, Argentina (2003-2011) . . . . . . 113

3.10. Indicadores alternativos de vulnerabilidad, Perú (2004-2010) . . . . . . . . . 113

3.11. Indicadores alternativos de vulnerabilidad, Nicaragua (1998-2006). . . . . . 114

3.12. Indicadores alternativos de vulnerabilidad, Chile (1996-2006). . . . . . . . 115 
ÍNDICE DE TABLAS 


\section{Índice de figuras}

1.1. Ejemplo hipotético de dos hogares con la misma media y distinta varianza en los resultados del bienestar. . . . . . . . . . . . . . . . . . 41

1.2. Ejemplo hipotético de dos hogares con la misma media y varianza, pero con distinta asimetría en los resultados del bienestar. . . . . . . . . . . . 51

3.1. Evolución de la pobreza y la vulnerabilidad, Argentina (2003-2011). . . . . 97

3.2. Intervalos de confianza normales de los índices de vulnerabilidad, Argentina $(2003-2011) \ldots \ldots \ldots \ldots \ldots \ldots \ldots$

3.3. Evolución de la pobreza y la vulnerabilidad, Perú (2004-2010) . . . . . . . . 100

3.4. Intervalos de confianza normales de los índices de vulnerabilidad, Perú (20042010). . . . . . . . . . . . . . . . . . . . 102

3.5. Evolución de la pobreza y la vulnerabilidad, Nicaragua (1998-2005). . . . . 103

3.6. Intervalos de confianza normales de los índices de vulnerabilidad, Nicaragua

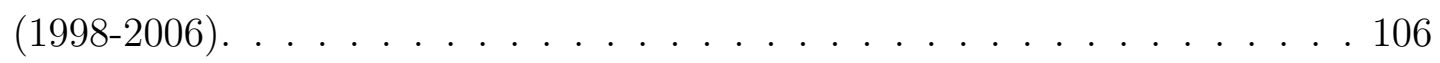

3.7. Evolución de la pobreza y la vulnerabilidad, Chile (1996-2006) . . . . . . . 107

3.8. Intervalos de confianza normales de los índices de vulnerabilidad, Chile $(1996-2006) \ldots \ldots \ldots \ldots \ldots$

3.9. Tasa de incidencia de la vulnerabilidad severa como proporción de la tasa de incidencia de la vulnerabilidad total. Relación con la tasa de incidencia de la pobreza. . . . . . . . . . . . . . . . . . . . 111

3.10. Intervalos de confianza percentiles de los índices de vulnerabilidad corregidos por sesgo, Argentina (2003-2011) . . . . . . . . . . . . 120

3.11. Intervalos de confianza percentiles de los índices de vulnerabilidad corregidos por sesgo, Perú (2003-2010) . . . . . . . . . . . . . . . . 121 
3.12. Intervalos de confianza percentiles de los índices de vulnerabilidad corregidos por sesgo, Nicaragua $(1998-2005) \ldots \ldots$. . . . . . . . . . . . 122

3.13. Intervalos de confianza percentiles de los índices de vulnerabilidad corregidos por sesgo, Chile (1996-2006). . . . . . . . . . . . . . . 123 


\section{Introducción}

El concepto de vulnerabilidad es uno de los más usados, pero a la vez, uno de los más controversiales en la literatura de pobreza. Es uno de esos conceptos acerca del cual todos intuyen lo que significa, pero sobre el cual, nadie es capaz de brindar una definición precisa que sea comúnmente aceptada. La medición de la vulnerabilidad, o más precisamente, de la vulnerabilidad a la pobreza, está plagada también de una diversidad de significados alusivos a este complejo fenómeno.

Por ello, las preguntas de investigación que se formulan en esta tesis doctoral, no son simples: ¿Qué es la vulnerabilidad a la pobreza? ¿Quiénes son las personas u hogares que deben ser considerados en tal condición de "vulnerables" frente al flagelo de la pobreza dentro de una población? Y una vez encontrados tales individuos vulnerables: ¿Cómo resumimos en una medida coherente cuanta vulnerabilidad a la pobreza existe en una población? Tras más de dos décadas de debate, en la literatura no existen respuestas claras a tales interrogantes, a pesar de que se han dedicado grandes esfuerzos intelectuales para tratar de resolverlas.

No se tiene acá la pretensión de brindar una respuesta unívoca y final a tales interrogantes. Pero se ha asumido con gran responsabilidad el desafío de abordarlas con profundidad, para tratar de brindar una contribución al debate. Al autor lo motiva fuertemente un desafío intelectual, pero más fuertemente el desafío moral de contribuir para que en Nicaragua ${ }^{1}$ y en América Latina, haya menos personas empobrecidas y viviendo en la amenaza de la incertidumbre sobre su presente y su futuro cercano. El resultado práctico de la solución a las interrogantes que se plantean en esta tesis es que quienes dirigen las políticas públicas puedan orientar mejor sus esfuerzos, en favor del bienestar de los que viven amenazados por la pobreza.

En la investigación también se enfrenta una interrogante empírica. Se pretende brindar

\footnotetext{
${ }^{1}$ El autor es originario de Nicaragua.
} 
una contribución sobre la reciente evolución de la vulnerabilidad a la pobreza en América Latina. Interesa saber además, si la reducción de la pobreza va siempre acompañada de reducciones en la vulnerabilidad, o si existen períodos en que la pobreza disminuye, pero en donde no obstante, permanece el riesgo de que las personas sigan viviendo en pobreza.

La disertación se encuentra organizada en tres capítulos, los cuales corresponden a su vez a tres artículos integrados en torno al mismo tema de estudio: la vulnerabilidad a la pobreza, con aplicaciones empíricas para América Latina. Los capítulos se desarrollan siguiendo muy de cerca el contenido de los artículos. Sin embargo, los títulos de los capítulos y de los artículos no se corresponden. El primer capítulo corresponde al artículo titulado: "Identificando la vulnerabilidad a la pobreza: una revisión crítica de la literatura". ${ }^{2}$ El segundo capítulo, en lo fundamental recoge el contenido del artículo: "Usando la semi-desviación típica de la media para medir la vulnerabilidad a la pobreza". ${ }^{3}$ Mientras que el tercer capítulo incorpora el artículo: "Midiendo la vulnerabilidad a la pobreza en América Latina". ${ }^{4}$

En el primer capítulo se enfrenta el problema de la identificación de la vulnerabilidad a la pobreza. Se realiza un análisis crítico del estado del arte respecto a la pregunta de quienes son aquellos que deben ser considerados vulnerables a la pobreza en una población. Se hace un esfuerzo por sintetizar más de dos décadas de debate en la literatura, para intentar así, encontrar algunas claves que permitan mejorar nuestra comprensión del fenómeno de la vulnerabilidad a la pobreza. Se concluye que el concepto en cuestión abarca dos dimensiones: (i) la pobreza esperada, como condición de que la pobreza sea un estado predecible dado el bienestar promedio del hogar, o (ii) el riesgo de caer en la pobreza, debido a los posibles desvíos de los resultados inciertos del bienestar bajo su valor esperado.

El segundo capítulo constituye el núcleo central de la tesis, y su principal contribución a la literatura. En dicho capítulo se utiliza un modelo de media-riesgo proveniente de la literatura de finanzas, para establecer un ordenamiento racional de los resultados del bienestar bajo condiciones de incertidumbre. Tal ordenamiento permite definir un criterio

\footnotetext{
${ }^{2}$ Este artículo y el tercero se encuentran en proceso de revisión para su publicación en revistas indexadas.

${ }^{3}$ Una versión concisa de este segundo capítulo ya fue publicada recientemente en la revista Economics Letters, bajo el título: "Using the downside mean-semideviation for measuring vulnerability to poverty".

${ }^{4}$ Este artículo fue premiado por la Academia Nacional de Ciencias Económicas de Argentina, como mejor trabajo inédito presentado en la XLVIII Reunión Anual de la Asociación Argentina de Economía Política.
} 
claro para identificar a los vulnerables a la pobreza a partir de la media del nivel de bienestar promedio y del riesgo de empeorar. Medido este último, como la desviación cuadrática promedio de las caídas bajo la media del bienestar (la semi-desviación típica a la baja). Se propone también una manera de agregar la vulnerabilidad en una medida resumen comúnmente aceptada en la literatura de pobreza y que se fundamenta en una base axiomática racional (una medida tipo FGT). Se propone además una distinción, nueva en la literatura, entre un tipo de vulnerabilidad determinada por el valor esperado del bienestar (la vulnerabilidad severa) y otro tipo de vulnerabilidad determinada por el riesgo (de naturaleza asimétrica) de caer en pobreza, debido a las desviaciones de los resultados inciertos del bienestar bajo tal media (la vulnerabilidad moderada). La medida propuesta se ilustra en un ejemplo empírico con datos de un panel de 2,561 hogares del Perú.

En el tercer capítulo se somete a un escrutinio empírico más riguroso y extensivo, la medida propuesta en el capítulo anterior. El nuevo indicador se calcula para un gran volumen de datos: 12 paneles de hogares de 4 países de América Latina. Se encuentra evidencia empírica de que el indicador resiste ser utilizado en tal diversidad de escenarios. También se somete el indicador a un extensivo ejercicio de remuestreo y se comprueba que su error estándar es pequeño. Se descubren además un par de hallazgos no menos relevantes: la vulnerabilidad no siempre desciende a la misma velocidad que la pobreza. Pueden haber períodos de descenso en la pobreza, acompañados de estancamientos en la disminución de la vulnerabilidad. Mientras que por otro lado, mayores índices de pobreza están vinculados a una mayor importancia relativa de la vulnerabilidad severa, en comparación con la vulnerabilidad moderada. Este segundo hallazgo constituye también una hipótesis nueva en la literatura: el desarrollo económico viene acompañado de un cambio en la preponderancia de la vulnerabilidad severa a la pobreza hacia la vulnerabilidad moderada. La consecuencia de ello para las políticas públicas es que los países más pobres deben focalizar esfuerzos para enfrentar la vulnerabilidad estructural y los países menos pobres deben incentivar mecanismos de seguros que permitan disminuir la incertidumbre de que los hogares obtengan resultados de desvíos a la baja con respecto a sus resultados esperados.

La contribuciones de esta tesis doctoral a la literatura, se indican en un breve apartado final, que sigue a las conclusiones de la disertación. 
El autor agradece a su hija Matilda y a su esposa Yana, por todo el tiempo que sacrificaron sin la presencia de su papá y de su esposo, para que él pudiera realizar esta investigación a la que dedicó cuatro años y medio de su vida adulta.

El autor agradece también a su director de tesis, el Dr. Luciano Di Gresia, por el apoyo brindado, y agradece también a los profesores que han sumado a su formación doctoral y a los que contribuyeron con sus críticas a mejorar los resultados de esta investigación. 


\section{Capítulo 1}

\section{El problema de la identificación}

\section{Resumen}

En la literatura económica sobre pobreza se han propuesto diversos índices para medir un fenómeno llamado "vulnerabilidad". Sin embargo, tras más de dos décadas de debate, aún no existe consenso sobre la cuestión más básica de como identificar cuales son los individuos que poseen tal condición de vulnerabilidad dentro de una población. En este capítulo realizamos una revisión crítica de los criterios de identificación de la vulnerabilidad a la pobreza existentes en la literatura hasta la primera mitad del año 2013, con la intención de acotar el debate y contribuir a acercar posiciones en la búsqueda de una síntesis conceptual. Se concluye que todos los enfoques de vulnerabilidad a la pobreza han enfatizado ya sea en el riesgo de caer en pobreza debido a la variabilidad de los resultados inciertos del bienestar, o en la pobreza esperada. Con algunos intentos por incorporar ambos elementos en un mismo enfoque.

\subsection{Introducción}

En un artículo seminal, Amartya Sen (1976), definió las dos interrogantes que se deben resolver para enfrentar el problema de la medición de la pobreza: " (i) identificar a los pobres entre la población total, y (ii) construir un índice de pobreza usando la información disponible sobre los pobres". A la primera cuestión se le conoce en la literatura de pobreza como "el problema de la identificación". A la segunda cuestión se le ha llamado "el problema de la agregación", o "de la medición" propiamente tal. No debería sorprendernos, 
si ambas cuestiones continuasen siendo válidas al enfrentar el problema de medición de la vulnerabilidad a la pobreza. Sin embargo, aunque en la investigación aplicada se han propuesto una serie de índices abocados a medir tal fenómeno, aún permanece sin esclarecerse la cuestión más básica de saber quienes son aquellos que deben ser considerados vulnerables a la pobreza dentro de una población.

En este capítulo realizamos un análisis crítico de los distintos criterios de identificación de la vulnerabilidad a la pobreza existentes en la actual literatura, con la finalidad de acotar el debate e intentar definir los puntos claves que caracterizan este fenómeno.

Otros estudios anteriores ya se han abocado a la tarea de revisar, tanto en términos conceptuales como metodológicos, los distintos enfoques de vulnerabilidad a la pobreza que han sido propuestos. El más conocido de estos trabajos de revisión ha sido sin duda el de Hoddinott y Quisumbing (2003). Otros autores que se han enfocado en un propósito similar son: Hoogeveen et al. (2004), Ligon y Schechter (2004), Gaiha e Imai (2009) y más recientemente Klasen y Povel (2013).

Lo que nos distingue de tales estudios es, que en primer lugar nos ajustamos fielmente a la separación de cuestiones indicada en Sen (1976), centrándonos solamente en el problema de la identificación. Dejamos así, para una etapa posterior, el problema de la agregación de la vulnerabilidad de una población en una medida resumen. Pensamos que analíticamente este es el camino adecuado a seguir, para tratar de aclarar el fenómeno de la vulnerabilidad a la pobreza y para poder medirlo luego de mejor manera. En segundo lugar, nuestro propósito no es solamente analizar críticamente los distintos enfoques existentes, sino buscar también sus aspectos comunes, para contribuir a acercarnos a una síntesis conceptual. En tercer lugar, ofrecemos una revisión más completa que las anteriores existentes en la literatura, al abarcar nueve enfoques existentes hasta la segunda mitad del año 2013. Finalmente, la estructura del capítulo ofrece una clasificación de enfoques de vulnerabilidad a la pobreza en dos vertientes principales, según el estado del arte hasta mediados del año 2013. La primera vertiente corresponde a los enfoques que enfatizan en el componente de riesgo de la vulnerabilidad. La segunda vertiente se focaliza en el componente de pobreza esperada, con algunos intentos por incorporar también el primer elemento. Desde nuestra perspectiva, esta clasificación viene a actualizar la que fue propuesta hace una década por Hoddinott y Quisumbing (2003).

Los nuevos enfoques de vulnerabilidad a la pobreza que se han incorporado en la 
literatura permiten también hacer referencia a nuevos conceptos, tales como: la vulnerabilidad a la pobreza relativa, la vulnerabilidad subjetiva a la pobreza y la vulnerabilidad multidimensional a la pobreza.

Vale indicar asimismo que alcanzar un acuerdo conceptual en este tema es de gran relevancia para las políticas públicas, para la cooperación internacional destinada al desarrollo y para la investigación económica aplicada. La falta de acuerdo acerca de que es la vulnerabilidad a la pobreza no solamente genera confusión y ambigüedad para el estudio de dicho fenómeno, sino que le quita a tal concepto gran parte de su relevancia heurística y de su utilidad práctica para fines de políticas públicas.

Para efectos prácticos, extender el análisis desde los conceptos de pobreza efectiva, hacia el ámbito más amplio de la vulnerabilidad a la pobreza, permite distinguir entre aquellos que son pobres, aquellos que se espera que sean pobres y los que están en riesgo de convertirse en nuevos pobres. La certeza de las decisiones públicas en materia de superación de la pobreza, dependerá crucialmente de la evaluación de tales circunstancias, las cuales atañen directamente al problema de identificación de la vulnerabilidad a la pobreza.

El ensayo se encuentra estructurado del siguiente modo: en las secciones 1.2 y 1.3 se presentan y discuten los enfoques de vulnerabilidad a la pobreza existentes en la actual literatura; en la sección 1.4 se definen las características del fenómeno y luego se incorporan las conclusiones correspondientes al final del capítulo.

\subsection{Vulnerabilidad como exposición al riesgo}

\subsubsection{Vulnerabilidad como exposición al riesgo de los hogares de bajos ingresos}

\section{El enfoque}

Los primeros acercamientos al concepto de vulnerabilidad a la pobreza se produjeron en el terreno de los hallazgos empíricos que evidenciaron la relación entre la pobreza y la exposición al riesgo que enfrentan los hogares de bajos ingresos. Ya en la década de los ochenta, diversas investigaciones revelaron que los hogares rurales de bajos ingresos experimentan cambios importantes en sus niveles de bienestar, debido a las fluctuaciones 
estacionales, los cambios del clima y las fluctuaciones de los precios (Chambers et al., 1981; Ahluwalia, 1985; Longhurst et al., 1986). Mientras que otras investigaciones (Levy, 1977; Gottschalk, 1982; Bane y Ellwood, 1986) hicieron notar la distinción entre un tipo de pobreza de carácter estructural, que se observa persistente en el tiempo (la pobreza crónica), y otro tipo de pobreza circunstancial, determinada por la variabilidad de los resultados del bienestar que experimentan los hogares de bajos ingresos a través del tiempo (la pobreza transitoria). En el contexto de tales investigaciones aflora el concepto de vulnerabilidad como exposición no asegurada frente al riesgo.

Una contribución seminal para abordar la relación entre pobreza y riesgo en ese entonces, fue el trabajo de Ravallion (1988). En dicho artículo se plantea formalmente la pregunta acerca de como se ven afectadas las medidas agregadas de pobreza absoluta, por el riesgo asociado a la variabilidad de los resultados del bienestar, que causan los shocks que enfrentamos bajo incertidumbre. Ravallion concluye que tal variabilidad provoca un incremento de la pobreza esperada, medida por los índices convencionales tipo FGT (Foster-Greer-Thorbecke, 1984) ó Atkinson (1987), siempre que se cumplan ciertas condiciones sobre la función de bienestar individual y sobre su distribución. ${ }^{1}$ Este autor no llamó entonces "vulnerabilidad" a tal relación entre la pobreza y el riesgo. Sin embargo, su contribución describió una relación entre la pobreza y la incertidumbre que con posterioridad empezó a llamarse "vulnerabilidad".

El término "vulnerabilidad" empieza a usarse en forma habitual en la literatura de pobreza durante la década de los noventa. Watts y Bolhe (1993) proponen el concepto de "espacio de vulnerabilidad" para referirse a una configuración histórica y local de pobreza y hambruna que afecta a una población. En tanto que un par de publicaciones destacadas en The American Economic Review utilizan el término vulnerabilidad en forma más decidida. El primero es el trabajo de Morduch (1994) sobre pobreza y vulnerabilidad, y el otro de Kochar (1995) sobre vulnerabilidad de los hogares a los shocks idiosincráticos de ingresos. En su artículo, Morduch introduce el concepto de "pobreza estocástica" para referirse a la situación en que un hogar obtiene resultados de consumo corriente bajo la línea de pobreza, a pesar de tener un ingreso permanente por encima de tal umbral. Este investigador también plantea la hipótesis de que tal situación de "vulnerabilidad" conduce

\footnotetext{
${ }^{1}$ Tales condiciones son las siguientes: (i) Que la función de bienestar individual sea cuasi-cóncava tanto en su argumento constante, como en su argumento aleatorio (el componente de riesgo) ; (ii) Que dicha función sea cóncava en su componente de riesgo y (iii) Que la línea de pobreza sea menor que la moda de la distribución del índice de bienestar.
} 
a decisiones de portafolio de segundo mejor, las cuales acarrean pérdidas de bienestar en comparación con lo que serían las decisiones óptimas de primer mejor, si existiesen los mecanismos adecuados de seguros y ausencia de restricciones de liquidez para los hogares de bajos ingresos. A partir de ello, la vulnerabilidad empieza a entenderse como la incapacidad de los hogares de bajos ingresos para suavizar su consumo a través del tiempo.

En otros trabajos de la década de los noventa se contrasta la hipótesis de exposición no asegurada de los hogares frente al riesgo (Cochrane, 1991; Townsend, 1994; Grimard, 1996; Ravallion y Chaudhuri, 1997; Jalan y Ravallion, 1999). Pero no es sino a partir de las contribuciones de Glewwe y Hall (1998), Dercon y Krishnan (2000) y Amin et al.(2003), cuando se acuña definitivamente el concepto de vulnerabilidad como riesgo que enfrentan los hogares debido a su incapacidad para absorber diversos shocks de ingresos a través del tiempo.

De acuerdo con este enfoque conceptual, para determinar si un hogar es o no vulnerable, debemos contrastar su exposición no asegurada frente al riesgo a través de un análisis de regresión que permita estimar el impacto de diversos shocks en los cambios del consumo. La forma de hacerlo fue propuesta primero por Cochrane (1991), para datos de corte transversal. Sin embargo, luego ha sido implementada preferentemente con datos de panel (Townsend, 1994; Ravallion y Chaudhuri, 1997; Jalan y Ravallion, 1999; Glewwe y Hall, 1999; Dercon y Krishnan, 2000; Tesliuc y Lindert, 2002; Amin el at., 2003; Skoufias y Quisumbing, 2005; entre otros). Especificaciones diversas se han propuesto, pero pueden resumirse en la ecuación básica que Hoddinott y Quisumbing (2003) han expresado como sigue:

$$
\Delta \ln c_{j t v}=\sum_{i=1}^{n_{1}} \alpha_{i} S(i)_{t v}+\sum_{i=1}^{n_{2}} \beta_{i} S(i)_{j t v}+\sum_{v=1}^{n_{3}} \delta_{v} D_{v}+\gamma X_{j v}+\Delta \varepsilon_{j t v}
$$

Donde, $\Delta \ln c_{j t v}$ es el cambio en el logaritmo del consumo per cápita o consumo equivalente del hogar $j$, ubicado en la localidad $v$, en el período $t ; S(i)_{t v}$ denota diversos shocks covariados que afectan a la localidad $v ; S(i)_{j t v}$ representa los shocks idiosincráticos que afectan al hogar; $D_{v}$ es una variable muda que toma el valor uno cuando el hogar pertenece a la localidad $v ; X_{j v}$ es un vector de características observables del hogar $j ; \mathrm{y} \Delta \varepsilon_{j t v}$ es el componente aleatorio de efectos no observables. Mientras que $\alpha_{i}, \beta_{i}, \delta_{v}$ y el vector $\gamma$, son los parámetros a estimar. 
Una forma simple de capturar el resumen de shocks covariados que afectan a cada localidad, es utilizar los cambios en el logaritmo de su ingreso medio: $\Delta \overline{\ln }_{t v}$. Aunque también es posible, en su lugar, incorporar en $S(i)_{t v}$ algunos shocks particulares conocidos, que afectan a cada comunidad. Por ejemplo los cambios en el clima que afectan las cosechas, o caídas en los precios agrícolas, cuando se trata de comunidades rurales.

Por su parte, los shocks idiosincráticos se pueden resumir de manera práctica a través del cambio en el logaritmo del ingreso de cada hogar: $\Delta \ln y_{j t v}$. O en su lugar, se pueden incorporar varios shocks específicos que afectan al hogar, tales como: enfermedades, pérdida de empleo del jefe de hogar, u otros.

Al tomar $\Delta \overline{\ln }_{t v}$ y $\Delta \ln y_{j t v}$, como medidas resúmenes de $S(i)_{t v}$ y $S(i)_{j t v}$ respectivamente, la ecuación (1.1) se puede estimar fácilmente a través de la siguiente especificación:

$$
\Delta \ln c_{j t v}=\alpha \Delta \overline{\ln }_{t v}+\beta \Delta \ln y_{j t v}+\sum_{v=1}^{k} \delta_{v} D_{v}+\gamma X_{j v}+\Delta \varepsilon_{j t v}
$$

Particular interés en este enfoque tiene el coeficiente $\beta$, el cual revelaría la capacidad para absorber los shocks idiosincráticos. Si los hogares se encuentra plenamente asegurados frente a shocks idiosincráticos, deberíamos obtener como resultado: $\beta=0$. En cambio, si se encuentran en total indefensión frente a shocks idiosincráticos, obtendríamos: $\beta=1$. Un problema de la especificación en (1.2) es que el coeficiente $\beta$ no brinda un criterio claro de identificación de la "vulnerabilidad" para cada hogar, sino solamente ofrece una medida de la "vulnerabilidad" promedio para la muestra, condicional a un set de variables de control. Ello puede arreglarse no obstante, siguiendo un diseño similar, pero con coeficientes específicos para cada hogar, como en la propuesta de Amin et al. (2003). De tal modo, es posible identificar la "vulnerabilidad" de cada hogar conforme el siguiente criterio de identificación que está implícito en la mayor parte de la literatura que sigue este enfoque y que se encuentra explícito en Amin et al. (2003: 60) del siguiente modo:

\section{Criterio 1 (Vulnerabilidad como exposición no asegurada frente al riesgo):}

Un hogar se define vulnerable si y solo si, no posee la capacidad de suavizar su consumo frente a las fluctuaciones idiosincráticas de sus ingresos.

La evidencia empírica ha mostrado que la exposición al riesgo idiosincrático es parcial. Es decir, las estimaciones han arrojado valores significativamente distintos de cero, pero 
menores que uno, para el coeficiente que mide la sensibilidad de los cambios en el consumo frente cambios idiosincráticos en el ingreso. Sin embargo, cuando se han implementado variantes de la regresión en (1.2) para grupos de hogares en forma separada, se ha encontrado evidencia de que los hogares de menores ingresos se encuentran más desprotegidos (son más "vulnerables") frente a shocks idiosincráticos, en comparación con aquellos de mayores recursos (ver por ejemplo: Jalan y Ravallion, 1999; Amin et al., 2003).

\section{Análisis crítico}

La exposición no asegurada frente al riesgo, efectivamente denota "vulnerabilidad", en el sentido de que constituye un estado de indefensión e inseguridad que por sí mismo genera una pérdida de bienestar.

Este enfoque tiene el mérito de haber levantado la cuestión de que uno de los aspectos centrales de la vulnerabilidad es el componente de riesgo. Sin embargo, tal y como lo han indicado diversos autores (Ligon y Schechter, 2002; Hoddinott y Quisumbing, 2003; Klasen y Povel, 2013) y como lo reconocen también Amin et al. (2003) la exposición no asegurada frente al riesgo puede afectar a hogares de cualquier nivel de bienestar. Es decir, este enfoque trata de un tipo de "vulnerabilidad" que no es exclusiva de los hogares que se encuentran expuestos a la pobreza, sino que atañe a un fenómeno más general. Muchos hogares de clase media, e inclusive de sectores de ingresos altos, serían también vulnerables desde esta perspectiva (como en efecto lo son), en la medida en que su consumo sea sensible a las fluctuaciones idiosincráticas de sus ingresos. Por ello, el tipo de vulnerabilidad que identifica este enfoque, no es exactamente vulnerabilidad a la pobreza, sino otra relacionada con la falta de instituciones adecuadas de seguros y de otros mecanismos de protección para el manejo de riesgos.

No obstante lo anterior, hay dos sentidos en que este enfoque de vulnerabilidad se ve vinculado directamente con el análisis de la pobreza. En primer lugar permite identificar una alta exposición al riesgo entre los hogares de bajos ingresos (Jalan y Ravallion, 1999; Amin et al., 2003; Dercon y Krihnan, 2000). Lo cual probablemente da cuenta de que éstos hogares son usualmente los que tienen menor capacidad para el manejo de riesgos. Ello nos permite hablar, dentro del marco del enfoque, de la "vulnerabilidad de los pobres" y de los que se encuentran al borde de la pobreza. En segundo lugar el enfoque da cuenta de que existen hogares que se encuentran en riesgo de caer en pobreza, justamente debi- 
do a tal incapacidad para suavizar su consumo a través de tiempo (Dercon y Krishnan, 2000). Entre estos hogares se encuentran por ejemplo, aquellos que son transitoriamente pobres. Desde nuestra perspectiva, es en este último sentido principalmente, que el enfoque en cuestión constituye un aporte importante al estudio de la vulnerabilidad a la pobreza. La exposición al riesgo es un componente fundamental para caracterizar el fenómeno de interés y que ineludiblemente está presente en los enfoques más recientes sobre vulnerabilidad a la pobreza.

Un aspecto de este enfoque de vulnerabilidad que ha sido cuestionado con toda razón en la literatura anterior (Hoddinott y Quisumbing, 2003; Povel, 2010) es que en algunas de sus versiones ha prevalecido una visión simétrica del riesgo como variabilidad y no como variabilidad a la baja. El coeficiente $\beta$ en (1.2), captura la sensibilidad de los cambios del consumo, respecto de los cambios en el ingreso corriente, sin diferenciar si tales cambios corresponden a caídas o a incrementos en el ingreso. En contraste, los enfoques más recientes sobre vulnerabilidad a la pobreza que se enmarcan en la perspectiva del riesgo, han enfatizado que éste es un fenómeno de naturaleza esencialmente asimétrica (Povel, 2010; Dutta et al, 2011; Klasen y Povel, 2013). De modo que, la dispersión de resultados de bienestar que debe interesarnos para la evaluación de la vulnerabilidad a la pobreza no es la dispersión per sé, sino solamente la dispersión a la baja, causada por shocks negativos.

\subsubsection{Enfoque de la línea de pobreza extendida}

\section{El enfoque}

La posibilidad de usar líneas de pobreza extendidas para identificar como vulnerables a aquellos que se encuentran apenas por encima de la línea de pobreza fue sugerida por Ravallion (1996: 1330), ${ }^{2}$ quien utiliza el término "vulnerables" para referirse a los que se encuentran en tal situación. Sin embargo, quienes desarrollaron este enfoque fueron Cafiero y Vakis (2006). La propuesta no tuvo gran acogida, ni dio pie a nuevas reformulaciones durante la década pasada, a pesar de que aparentemente ofrece ventajas de simplicidad conceptual.

El enfoque consiste en identificar a los vulnerables redefiniendo simplemente la línea

\footnotetext{
${ }^{2}$ Aunque al parecer Ravallion toma la idea de Lipton (1983) quien ya había sugerido usar varias líneas de pobreza para capturar diversos niveles de privación.
} 
de pobreza, de manera que ésta incorpore el costo de un seguro, que garantice alcanzar tal umbral con una cobertura de riesgo socialmente aceptable. En palabras de Cafiero y Vakis (2006), el umbral de vulnerabilidad "es la línea de pobreza que incluye el monto de consumo requerido para satisfacer las necesidades básicas, más el costo necesario para adquirir un seguro suficiente". De acuerdo con ello, "un seguro suficiente" es aquel que cubre una exposición al riesgo considerada socialmente aceptable. ${ }^{3}$

Formalmente, la línea de pobreza extendida puede expresarse como: $z+\eta_{j}$, donde $z$ es la línea de pobreza absoluta convencional y $\eta_{j}$ es el costo de un seguro específico "socialmente aceptable" para que el hogar o individuo $j$ elimine la incertidumbre para un nivel de bienestar igual a $z$. La consecuencia directa de tal definición es que la brecha de pobreza individual incorpora tal cobertura de riesgos del siguiente modo:

$$
\widetilde{g}_{j}=\left(z+\eta_{j}\right)-y_{j}
$$

donde $y_{j}$ es la variable focal con que se mide la pobreza.

Dado $\widetilde{g}_{j}$, la pobreza extendida, que tiene en cuenta la pérdida de bienestar por exposición al riesgo (la vulnerabilidad), puede medirse directamente a nivel agregado, utilizando los índices convencionales de pobreza tipo FGT:

$$
P_{\alpha}(\mathbf{y}, \eta, \mathrm{z})=\frac{1}{N} \sum_{i=1}^{q}\left(\frac{\widetilde{g}_{j}}{z+\eta_{j}}\right)^{\alpha}, \alpha \geq 0
$$

Donde según lo acostumbrado, $\mathbf{y}$ es el vector que contiene los valores $y_{j}$ de toda la población, ordenados de menor a mayor, pero incluyendo el seguro de riesgo $\eta_{j}$. Mientras tanto, $\eta$ es el vector que contiene los correspondientes valores $\eta_{j}$ de la población, ordenados en correspondencia con los $y_{j} ; N$ es el tamaño de la población, $q$ es el máximo valor de $\widetilde{g}_{j}$, tal que $\widetilde{g}_{j} \leq z$, y $\alpha$ es el parámetro de aversión a la pobreza.

El criterio de identificación de la vulnerabilidad a la pobreza que sigue este enfoque puede interpretarse del siguiente modo:

Criterio 2 (Vulnerabilidad como línea de pobreza extendida): Sea $y_{j}$ la variable focal utilizada para medir la pobreza de una población, sea z el umbral de pobreza bajo

\footnotetext{
${ }^{3}$ La traducción del autor es literal. El término: "enough insurence" quizás podría ser interpretado de mejor manera como un "seguro adecuado", o de "suficiente cobertura" para garantizar el monto de consumo requerido para satisfacer las necesidades básicas con un riesgo socialmente aceptable.
} 
certidumbre, sea $\eta_{j}$ el costo de un seguro que eliminaría el riesgo de que la variable aleatoria $y_{j}$ se desvíe de $z$, cuando $y_{j}$ es igual a $z$. Entonces un hogar se define vulnerable a la pobreza si y solo si $y_{j} \leq z+\eta_{j}$.

\section{Análisis crítico}

Una debilidad importante de este enfoque, más bien de tipo práctico, es la complejidad que presenta la estimación de $\eta_{j}$. Se requiere estimar tantos $\eta_{j}$ como individuos u hogares hayan en la población considerada (o en la muestra representativa utilizada). Cafiero y Vakis (2006) no aclaran este punto. El problema no es trivial, teniendo en cuenta que cada individuo u hogar podría tener un riesgo distinto para alcanzar el umbral $z$.

En la práctica habría que calcular $N$ líneas de pobreza, lo cual dificulta la comprensión del enfoque. Las brechas de pobreza de cada individuo u hogar no serían comparables, debido a las diferencias en los umbrales de pobreza individuales. Tampoco serían comparables las brechas de pobreza entre grupos, porque estos tendrían también distintos umbrales.

Por otro lado, el enfoque no se sustenta en un modelo aceptado de ordenamiento de los resultados del bienestar bajo incertidumbre, sino más bien un ordenamiento de los umbrales de pobreza a partir de los requerimientos individuales de un seguro. Desde nuestra perspectiva, para explicar un fenómeno como la vulnerabilidad a la pobreza, que está ligado a la incertidumbre, es necesario valerse necesariamente de algún modelo válido para comparar resultados aleatorios del bienestar (dominancia estocástica, dominancia en media riesgo o utilidad esperada).

Sin embargo, a pesar de tales limitaciones, el enfoque representa un aporte, al sugerir la idea de incorporar de algún modo un parámetro de riesgo dentro del índice FGT, para tener en cuenta la incertidumbre dentro de la medida de pobreza, capturando así la vulnerabilidad debida al riesgo. ${ }^{4}$

\footnotetext{
${ }^{4}$ Tal idea había sido señalada ya antes por Ravallion (1988), pero dentro de un enfoque agregado. La idea es retomada luego en Gallardo (2013), pero replanteada en el marco de un modelo de dominancia en media riesgo, en donde el ordenamiento bajo incertidumnbre corresponde a los resultados de bienestar y no a las líneas de pobreza.
} 


\subsubsection{La Propuesta de Béné}

\section{El enfoque}

Christohe Béné (2009) propuso una medida de vulnerabilidad a la pobreza fundada en el coeficiente de variación. El componente básico del índice de Béné, es la siguiente expresión, tomada de Devereux (2001):

$$
V_{j g}=C V_{g} D e p_{j a}
$$

En donde $V_{j g}$ denota el índice de vulnerabilidad del hogar $j ; C V_{g}$ es el coeficiente de variación de los hogares del grupo $g$, al cual pertenece el hogar $j$; mientras que $D e p_{j a}$ es "la tasa de dependencia de ingresos en efectivo" del hogar $j$, medida como la proporción de ingresos en efectivo que obtiene este hogar en su actividad principal $a$. Por ejemplo, si en su actividad principal el hogar depende solamente de un salario monetario en efectivo, su tasa de dependencia de ingresos en efectivo será igual a uno. En cambio, si depende de ingresos de capital de los cuales obtiene solo una proporción en efectivo, su tasa de dependencia de efectivo será menor que uno.

El sentido de la medida de Devereux (2001) es que el término $C V_{g}$, capturaría los shocks covariados (efectos de grupo) que afectan al hogar, mientras que $D e p_{i a}$ capturaría la vulnerabilidad de tipo indiosincrática. Al igual que Devereux (2001), Béné (2009) considera que la vulnerabilidad se compone justamente de estos dos elementos. El primero de ellos, según él, representa la exposición al riesgo propiamente tal y el segundo representa la susceptibilidad o incapacidad individual de poder enfrentar tales shocks.

Sin embargo, Béné no se muestra satisfecho con el índice expresado (1.5), ya que dicho indicador no toma en consideración la atenuación del riesgo por diversificación. Así, de acuerdo con (1.5), dos hogares que pertenecen al mismo grupo serían considerados igualmente vulnerables, si en su actividad principal generaran el mismo porcentaje de ingresos en efectivo, aunque uno de ellos estuviese diversificado en varias actividades, o realizase otras actividades de subsistencia que le generasen ingresos no monetarios (por ejemplo: ingresos en especies para el auto-consumo producto de cultivos y crianza de animales). Por ello, Béné introduce en (1.5), el siguiente índice de diversificación de actividades:

$$
D i v_{j}=A_{j}\left(1-D e p_{j a}\right)+\sqrt{S u b_{j}+1}
$$


Donde $A_{j}$ es el número total de actividades económicas para la generación de ingresos en las que participa el hogar $j$. Mientras que $S u b_{j}$ es la proporción de actividades de subsistencia dentro del número total de actividades. Acá, el término $A_{j}\left(1-D e p_{j a}\right)$ captura la diversificación por fuentes de ingresos, mientras que $\sqrt{S u b_{j}+1}$, captura la diversificación por concepto de actividades de subsistencia que generan ingresos en especie. ${ }^{5}$ Como resultado, Béné obtiene la siguiente modificación de (1.5):

$$
V_{j g}=C V_{g} \operatorname{Dep}_{i a} \frac{1}{\operatorname{Div}_{j}}
$$

Para completar su indicador, Béné hace la conexión de la vulnerabilidad con la pobreza, incorporando al índice en (1.7), un componente de brecha de pobreza definido como la raíz cuadrada de su recíproco: $P o v_{j}=\sqrt{\frac{z}{z_{j}}}$, donde $z$ es la línea de pobreza y $z_{j}$ es el ingreso monetario del hogar $j$. Obteniendo así, la siguiente expresión final, para la vulnerabilidad a la pobreza del hogar $j$ :

$$
V_{j g}=C V_{g} \operatorname{Dep}_{i a} \frac{1}{\operatorname{Div}_{j}} \operatorname{Pov}_{j}
$$

La inclusión de $\mathrm{Pov}_{j}$ en el indicador es justificada por este autor, al considerar que los hogares más pobres son más vulnerables, y que aquellos que están por encima de la línea de pobreza a una distancia mayor lo son menos. ${ }^{6}$

El criterio de identificación de la vulnerabilidad que se deduce de la propuesta de Béné, es el siguiente:

Criterio 3 (Coeficiente de variación y dependencia de efectivo): Sea $C V_{g}$ el coeficiente de variación de los hogares del grupo g, al cual pertenece el hogar $j$. Sea Dep ja la tasa de dependencia de efectivo del hogar $j$, medida como proporción de ingresos en efectivo que obtiene $j$ de su actividad principal a. Entonces, el hogar $j$ es vulnerable a la pobreza si y solo si: $C V_{g}>0$ y Dep ja $>0$.

\footnotetext{
${ }^{5}$ Béné dice utilizar la raíz cuadrada en esta expresión porque supone un efecto marginal positivo decreciente de las actividades de subsistencia sobe la vulnerabilidad de los hogares. Además agrega la constante 1, para poder computar $D i v_{j}$ en el caso en que $D e p_{j a}=1$ y $S u b_{j}=0$

${ }^{6} \mathrm{La}$ inclusión de la raíz cuadrada en la definición de $P o v_{j}$ nuevamente está asociada al supuesto de un efecto marginal decreciente de la pobreza sobre la vulnerabilidad.
} 


\section{Análisis crítico}

La propuesta de Béné (2009) no es propiamente un enfoque de vulnerabilidad como riesgo de caer en pobreza, sino de vulnerabilidad como estado general de indefensión económica, dentro del cual la pobreza es uno de sus componentes constitutivos. En nuestra interpretación, el elemento central de la vulnerabilidad en esta propuesta, es claramente la exposición al riesgo, mientras que la pobreza se incorpora como un elemento agravante.

La principal debilidad del índice de Béné es su alto grado de complejidad. Se trata de un índice difícil de calcular para la población entera de un país, ya que requiere mucho detalle de información. Sin embargo puede ser un enfoque de gran utilidad para la realización de estudios focalizados en pequeñas comunidades. ${ }^{7}$

El mérito de Béné es poner en la discusión una serie de elementos que están vinculados con la vulnerabilidad a la pobreza y que antes no habían sido mencionados. Los elementos de riesgo y de brecha de pobreza, si bien ya se conocían y ya se habían combinado antes en la literatura de vulnerabilidad a la pobreza cuando apareció este enfoque (Ligon y Schechter, 2003; Calvo y Dercon, 2005 y 2007b), no habían sido sin embargo vinculados a características como la diversificación de cartera o a estadísticas resúmenes de riesgo, como el coeficiente de variación. En tal sentido, el trabajo de Béné (2009) es un aporte a la discusión que da luces para pensar en el problema desde la perspectiva de los desarrollos de la literatura de riesgo en las finanzas y desde la perspectiva de medidas estadísticas resúmenes.

\subsubsection{Vulnerabilidad como percepción subjetiva del riesgo a la baja}

\section{El enfoque}

Como se indicó antes, una de las principales limitaciones de los enfoques tempranos de vulnerabilidad focalizados en el componente de riesgo, era su visión simétrica del mismo. No obstante, dentro de las propuestas que siguen esta línea conceptual, tal limitación es superada en el trabajo de Povel (2010) y luego en Dutta et al. (2011). ${ }^{8}$

\footnotetext{
${ }^{7}$ La aplicación empírica del artículo de Béné (2009) por ejemplo corresponde a una comunidad de 74 hogares de pescadores.

${ }^{8}$ Este es un punto muy relevante que ya había sido antes levantado entes desde otra línea de investigación por Calvo (2005 y 2007), al indicar que los resultados del bienestar por encima de la línea de pobreza no son relevantes para la evaluación del riesgo de caer bajo tal umbral.
} 
La propuesta de Povel (2010), ${ }^{9}$ sigue la definición del Banco Mundial (World Bank, 2001), según la cual: "la vulnerabilidad mide la capacidad de resistencia contra un shock la probabilidad de que un shock resulte en una disminución en el bienestar". ${ }^{10}$ De acuerdo con ello, se desarrolla un concepto general de vulnerabilidad con respecto del statu quo del bienestar y no precisamente con respecto a la pobreza. La vulnerabilidad es entendida como el riesgo de obtener pérdidas futuras de bienestar.

En el concepto de Povel (2010: 3), "el riesgo es un evento negativo futuro cuya ocurrencia tiene cierta probabilidad". ${ }^{11}$ Por ello, las posibles mejoras de bienestar no son relevantes para su evaluación. Además, de acuerdo con este enfoque, la vulnerabilidad es un concepto que "mira hacia adelante", en el sentido que compete solamente a potenciales eventos futuros. En este último aspecto Povel (2010) levanta críticas contra los enfoques anteriores de vulnerabilidad, indicando que estos utilizan en forma incorrecta la información del pasado para proyectar los eventos del futuro. De acuerdo con su visión, ello no tiene en cuenta que los agentes dirigirán sus estrategias en el futuro, para evitar o manejar mejor los riesgos, utilizando las experiencias del pasado. Así que no tendríamos por qué esperar que los mismos eventos desafortunados se repitan.

Para poder pronosticar los riesgos que enfrentan los hogares, de manera que sea "mirando hacia adelante" y no "mirando hacia atrás", ${ }^{12}$ el autor propone utilizar información subjetiva de lo que los propios agentes esperan de su futuro. Así, en su aplicación empírica, Povel (2010) utiliza datos de encuestas de hogares destinadas a recopilar información sobre shocks que los hogares esperan enfrentar en el futuro y sobre sus correspondientes probabilidades.

La propuesta de Povel (2010) para la medición de la vulnerabilidad consiste en asignar a cada hogar $h$ un índice de privación $d_{j i} \in[0,1]$, para cada estado de naturaleza $i$, con probabilidad $p_{j i}$, en los $n$ estados de naturaleza futuros. El índice de vulnerabilidad para cada hogar $j$, de acuerdo a este enfoque, se define como:

$$
V_{j}=\sum_{s=1}^{n} p_{j i} d_{j i}^{\alpha}
$$

\footnotetext{
${ }^{9}$ Véase también: Klasen y Povel (2013).

${ }^{10}$ La traducción es del autor. El texto original en inglés es: "vulnerability measures the resilience against a shock - the likelihood that a shock will result in a decline in well-being".

${ }^{11}$ La traducción es del autor. El texto original en inglés es el siguiente: "Risk is a negative future event whose occurrence has a certain probability".

${ }^{12}$ Se utilizan los términos "mirando hacia adelante" y "mirando hacia atrás", como traducciones del inglés de "forward looking" y "backward looking".
} 
Donde, $0 \leq d_{j i} \leq 1, \sum_{s=1}^{n} p_{j i}=1$, y $\alpha$ es un parámetro de riesgo. Con aversión al riesgo se tiene que: $\alpha>1$. Tanto en Povel (2010), como en el reciente libro de Klasen y Waibel (2013), se ofrece evidencia empírica de implementación de esta medida con datos de encuestas de hogares de Thailandia y Vietnam.

El criterio de identificación de la vulnerabilidad de este enfoque, puede definirse como sigue:

\section{Criterio 4 (Vulnerabilidad como percepción subjetiva del riesgo a la baja):}

Sea $d_{j i} \in[0,1]$, un índice de privación que representa una posible pérdida futura en el bienestar del hogar $j$ respecto de su bienestar actual, en el estado de naturaleza i; sean: $p_{1}, p_{2}, \ldots, p_{n}$, las probabilidades de los $n$ estados de naturaleza futuros. Entonces, el hogar $j$ es vulnerable, si y solo si $V_{j}=\sum_{s=1}^{n} p_{j i} d_{j i}>0$.

Por supuesto el criterio anterior no requiere incluir el parámetro de riesgo $\alpha$, ya que, para que la expresión (1.9) sea mayor que cero, es requisito que $V_{h}=\sum_{s=1}^{n} p_{h i} d_{h i}$, también lo sea.

\section{Análisis crítico}

Hay tres aportes importantes a destacar en este enfoque. El primero de ellos es que supera la visión simétrica del riesgo, que caracterizó a algunos planteamientos previos dentro de esta línea conceptual. El enfoque se construye a partir de un índice de privación que solo considera relevantes las desviaciones a la baja con respecto al nivel de bienestar vigente. Las desviaciones al alza están excluidas de la medida de vulnerabilidad. Un segundo aporte, es plantear por primera vez en la literatura, un concepto de vulnerabilidad subjetiva. Ello por supuesto es relevante, tal como lo es en la literatura de pobreza el concepto de pobreza subjetiva. Un tercer aporte, es plantear una visión de vulnerabilidad relativa, que trasciende la visión de la línea de pobreza absoluta como umbral de vulnerabilidad, dirigiendo la atención hacia otros umbrales posibles, cuando el propósito es investigar solamente el componente de riesgo de este fenómeno.

Sin embargo, nótese que este no es un enfoque de vulnerabilidad a la pobreza propiamente tal, puesto que se trata de un concepto de vulnerabilidad como riesgo de sufrir caídas desde cualquier nivel de bienestar, que no necesariamente conducen a situaciones de 
pobreza. Ello es reconocido tanto en Povel (2010), como en Klasen y Povel (2013), cuando hacen la distinción entre los conceptos de 'vulnerabilidad' y 'vulnerabilidad a la pobreza'. Ellos adhieren claramente al primer concepto, mientras que el segundo lo vinculan con la propuesta de Calvo y Dercon (2005 y 2007b), que analizaremos más adelante.

Nótese que en este enfoque se podrían considerar 'vulnerables', incluso a individuos que viven en la extrema riqueza, debido a que entre estos encontraremos algunos que esperan tener privaciones futuras de bienestar con cierta probabilidad mayor que cero.

Por supuesto el enfoque se puede vincular también a la pobreza, en el mismo sentido que el enfoque analizado en la subsección 1.2.1.. Esto es, cuando el enfoque se aplica a hogares de bajos ingresos, particularmente a los pobres y a los que están al borde de la línea de pobreza.

Por otro lado, este enfoque comparte una limitación común a las visiones de pobreza subjetiva, en relación a que el estado objetivo de bienestar puede estar desalineado con el subjetivo. Obsérvese que de acuerdo con este enfoque, hasta un afortunado millonario que peque un poco de pesismista puede ser considerado 'vulnerable'. Puesto que este, en sus pronósticos subjetivos respecto de los sucesos futuros, tendrá en mente una pérdida de bienestar. Mientras que un indigente optimista que espere una mínima mejora futura en su bienestar, sería considerado 'no vulnerable'. ${ }^{13}$

Otro aspecto criticable de este enfoque y que ha sido común a otros conceptos de vulnerabilidad que veremos más adelante, es el de asumir que la característica ex ante atribuible al fenómeno, necesariamente tiene relación con el pronóstico de potenciales situaciones futuras. Desde nuestra perspectiva, tal característica ex ante de la vulnerabilidad tiene relación solamente con que esta corresponde a un fenómeno de naturaleza aleatoria, que se experimenta ex ante, solo en el sentido de que es independientemente de como se revele particularmente la incertidumbre en un momento dado del tiempo. Para explicar mejor esto, supongamos que la vulnerabilidad de un hogar en cada período del tiempo en el intervalo $\left[t_{0}, T\right]$, depende de dos estados: $A$ y $B$, con igual probabilidad. Supongamos que $A$ es el 'estado bueno' en el cual el hogar no es pobre y que $B$ es el 'estado malo' en el cual el hogar se encontraría en pobreza. Para un hogar campesino, el estado $B$ podría ser por ejemplo un nivel de precipitaciones desfavorable para la cosecha (sequía o exceso de lluvias). En cambio, para un hogar urbano, el estado $B$ podrías ser

\footnotetext{
${ }^{13}$ Volveremos a insistir sobre este punto más adelante.
} 
por ejemplo, que las ventas de su pequeño negocio de subsistencia, caigan bajo cierto nivel. La condición ex ante de la vulnerabilidad tiene que ver con que esta no se alivia o aminora en $t$, cuando constatamos ex post que ha ocurrido $A$. Pues en el mismo período se enfrentó la amenaza de que ocurriera $B$. Sin embargo, la vulnerabilidad que enfrenta el hogar en $t$, puede evaluarse tanto antes de dicho período, a través del pronóstico de lo que va a ocurrir en el futuro, como también después de que transcurra $\left[t_{0}, T\right]$, al analizar la naturaleza del proceso estocástico que se reveló en los resultados generados. La naturaleza ex ante del fenómeno (su condición incierta) no desaparece cuando se evalúa la incertidumbre a partir de los resultados efectivos ya ocurridos.

Por otra parte, el argumento de que los individuos ajustan su comportamiento a partir de lo ocurrido en el pasado, debe considerar además que en el mismo pasado los agentes se comportaron en forma optimizadora y que por lo tanto, ya en el pasado eligieron la opción que más les convenía para el manejo de riesgos. Lo que no pueden cambiar en el futuro, es precisamente la incertidumbre objetiva que tampoco en el pasado podían controlar. Por ejemplo: si llueve o no llueve, si otros me compran lo que vendo o si no me lo compran, etcétera. Es decir, lo que no pueden cambiar es la naturaleza del proceso estocástico que enfrentan y que permanece cuando los agentes se enfrentan a las mismas condiciones objetivas. Es decir, cuando viven en el mismo ambiente, con los mismos recursos y expuestos a los mismos shocks.

\subsubsection{Enfoque de Dutta-Foster-Mishra}

\section{El enfoque}

Uno de los enfoques más recientes, que pone el énfasis en el elemento de riesgo, es la propuesta que desarrollaron Dutta et al. (2011). Al igual que Povel (2010), estos autores consideran que la vulnerabilidad tiene que ver con el riesgo de caer bajo cierto umbral de bienestar. Tal umbral puede corresponder a diferentes dimensiones, como la salud, la alimentación, el ingreso, etcétera. Abriéndose así, con tal visión, a la posibilidad de estudiar la vulnerabilidad desde una perspectiva multidimensional, tal y como fuera indicado antes que ellos por Calvo (2008). No obstante estos autores se focalizan en la vulnerabilidad de ingresos vinculada solo en forma relativa a la pobreza. Según ellos, la vulnerabilidad a la pobreza es un concepto al cual deben atribuirse las características que se indican a continuación. 
En primer lugar enfatizan que la vulnerabilidad está vinculada con una situación ex ante, acerca de una potencial privación de bienestar futura. En tal sentido también relacionan la vulnerabilidad con un pronóstico e indican que el concepto es dependiente del horizonte de predicción. Para desarrollar su propuesta deciden tomar un horizonte de predicción de un período solamente. Desde su perspectiva no obstante, la vulnerabilidad a la pobreza es un concepto distinto al de pobreza esperada que analizaremos más adelante y en donde el elemento característico será la predicción de alguna medida de pobreza. Acá en cambio, la figura clave es el potencial déficit de bienestar, que se genera al caer bajo un umbral de referencia híbrido, determinado tanto por la línea de pobreza, como por el ingreso inicial.

En segundo término, al igual que Povel (2010), indican que la vulnerabilidad está determinada esencialmente por el riesgo a la baja. Es decir, se trata de un concepto asociado al riesgo de caer bajo cierto umbral y por lo tanto no atañe a los estados contingentes que significan ganancias de bienestar.

En tercer lugar, también siguen a Povel (2010), al considerar que la vulnerabilidad es un concepto con especificidad individual. Pues cada individuo tiene una visión distinta del riesgo y porque además, iguales caídas de ingresos pueden reflejar distintos niveles de vulnerabilidad, en dependencia de cuales sean las capacidades individuales para el manejo de riesgos. Tales capacidades dependen según estos autores, de los niveles iniciales de ingresos. Para ellos es central considerar que los estándares de vida mínimos para cada individuo son diferentes y dependientes no solamente de la línea de pobreza, sino también del statu quo inicial. Los niveles iniciales de bienestar reflejan además la capacidad individual para responder a las caídas eventuales en los ingresos. Ya que las personas de mayores niveles de ingresos poseen una mayor dotación de activos y mayores redes de apoyo para protegerse de eventuales cambios desafortunados. Pero además, porque una caída en el bienestar por debajo de la línea de pobreza, genera una pérdida mayor, entre mayor sea el nivel de ingreso inicial.

En el enfoque se supone un conjunto finito de ingresos futuros $\left\{y_{t+1}^{1}, y_{t+1}^{2}, \ldots, y_{t+1}^{n}\right\}$, con probabilidades $p=\left\{p_{1}, p_{2}, \ldots, p_{n}\right\}$, tales que $\sum_{s=1}^{n} p_{s}=1$. Para la medición de la vulnerabilidad solamente se consideran los individuos para los que existen probabilidades mayores que cero de obtener resultados futuros por debajo de la línea de pobreza $z$. El potencial déficit de bienestar futuro es representado a través de una función de privación: 
$d\left(z, y_{t}, y_{t+1}\right)$, que cumple tres propiedades: (i) $d\left(z, y_{t}, y_{t+1}\right) \geq 0$; (ii) $d\left(z, y_{t}, y_{t+1}\right)$ es continua en $y_{t}, y_{t+1}$ y $z$; y (iii) para cualquier $\theta, \lambda>0$ y $d\left(z, y_{t}, y_{t+1}\right)>0$, se cumple que: $d\left(z+\theta, y_{t}, y_{t+1}\right)>d\left(z, y_{t}, y_{t+1}\right)$ y $d\left(z, y_{t}, y_{t+1}\right)>d\left(z, y_{t}, y_{t+1}+\lambda\right)$. Lo último implica, que la privación es mayor a mayor línea de pobreza y menor, a mayor ingreso futuro.

La función de privación en cada estado de naturaleza, $s=1,2, . . n$, es definida como:

$$
d\left(z, y_{t}, y_{t+1}^{s}\right)=R\left(z, y_{t}\right)-y_{t+1}^{s}
$$

Donde $R\left(z, y_{t}\right)$ es la línea de referencia a partir de la cual se miden las caídas de bienestar. Pero a diferencia de otros enfoques de vulnerabilidad a la pobreza, el umbral de referencia no es acá la línea de pobreza, sino una medida que toma en cuenta también el nivel de ingreso inicial $y_{t}$. Para efectos prácticos, $R\left(z, y_{t}\right)$, viene a ser una media ponderada entre $z$ e $y_{t}$. De modo que se trata de un enfoque híbrido de vulnerabilidad, que mezcla un umbral absoluto (la línea de pobreza), con uno relativo (el nivel de ingreso inicial).

Se supone que el individuo vulnerable enfrenta una lotería de privación:

$$
L=p_{1}, d\left(z, y_{t}, y_{t+1}^{1}\right) ; \ldots ; p_{n} d\left(z, y_{t}, y_{t+1}^{n}\right)
$$

A partir de ello, la clase general de medidas de vulnerabilidad que proponen Dutta et al. (2011) es la siguiente:

$$
V(L)=\sum_{s=1}^{n} p_{s} f\left(R\left(z, y_{t}\right)-y_{t+1}^{s}\right)
$$

Donde $f($.$) debe ser una función convexa, para asegurar que la medida representada$ en (1.12) cumpla con una base axiomática que abarca lo siguiente:

1. Descomposición: Para cualquier par de loterías de privación $L$ y $L^{\prime}$, y $0 \leq \alpha \leq 1$, se cumple que, $V\left(\alpha L+(1-\alpha) L^{\prime}\right)=\alpha V(L)+(1-\alpha) V\left(L^{\prime}\right)$.

2. Transferencia: Si de cualquier lotería de privación $L$, se deriva una nueva lotería $L^{\prime}$, tal que, en $L^{\prime}$ se ha transferido un monto de ingresos $t$ desde un estado de naturaleza peor (con mayor privación), hacia otro estado de naturaleza mejor (con menor privación) pero con igual probabilidad, entonces, $V\left(L^{\prime}\right)>V(L)$.

3. Monoticidad en los ingresos futuros: Si de cualquier lotería de privación $L$, se deriva una nueva lotería $L^{\delta_{i}}$, tal que, en $L^{\delta_{i}}$ es una lotería en la cual a uno de los ingresos 
futuros con probabilidad $p_{i}>0$ se le ha sumado el monto $\delta_{i}>0$, entonces, $V(L)>$ $V\left(L^{\delta_{i}}\right){ }^{14}$

4. Monoticidad en el nivel de bienestar inicial: Si a partir de cualquier lotería de privación $L$ se deriva una nueva lotería $L^{\delta}$, tal que $L^{\delta}$ se diferencia de $L$, solo porque se ha sumado el monto de ingresos $\delta>0$ a sus funciones de privación en todos los estados, entonces, para todo $\delta$ y $L$ se tiene que $V(L) \geq V\left(L^{\delta}\right)$, ó para todo $\delta$ y $L$ se tiene que $V(L) \leq V\left(L^{\delta}\right)$.

5. Independencia: Sea la lotería de privaciones igualmente distribuidas: $L_{E}=p_{1}, \bar{d} ; \ldots ; p_{n} \bar{d}$, tal que $\bar{d}=\sum_{s=1}^{n} \frac{d\left(z, y_{t}, y_{t+1}^{s}\right)}{n}$. Si para cualquier par de loterías $L$ y $L^{\prime}$, tal que $L_{E} \geq L_{E}^{\prime}$, y para algún $\delta>0: d\left(z, y_{t}, y_{t+1}^{i}+\delta\right)>0, d\left(z^{\prime}, y_{t}^{\prime}, y_{t+1}^{\prime i}+\delta\right)>0$ y $p_{i}>0$, entonces, $L_{E}^{\delta} \geq L_{E}^{\prime \delta}$.

Los primeros tres axiomas son de fácil interpretación ya que derivan directamente de la base axiomática común a los índices de pobreza (véase Foster et al., 2010). Sin embargo, los axiomas 5 y 6 requieren aclaración. El significado del axioma 4 deriva de que en este enfoque, el statu quo inicial puede estar correlacionado tanto positiva como negativamente con la vulnerabilidad. Es decir, al aumentar el statu quo en una cuantía $\delta$, la vulnerabilidad podrá ya sea inambiguamente tender a disminuir en comparación con el statu quo inicial, o podrá tender inanbiguamente a aumentar. Por ejemplo, si el statu quo inicial es mayor que la línea de pobreza y al aumentarlo en un $\delta>0$, sigue siendo probable caer bajo $z$, entonces, la privación de caer bajo la línea de pobreza y por lo tanto la vulnerabilidad, será mayor con $y_{t}+\delta$ que con $y_{t}$. Sin embargo, con un statu quo inicial, tal que, al agregarle $\delta$ desaparece la amenaza de caer bajo la línea de pobreza en algunos estados, entonces por el contrario, la vulnerabilidad será menor con $y_{t}+\delta$ que con $y_{t}$. Es decir, el statu quo inicial por un lado puede actuar como un elemento protector, pero por otro lado puede actuar como un elemento que marcaría una mayor pérdida de bienestar si se cae bajo la línea de pobreza.

En relación al axioma 5, lo que dice es que el ranking entre loterías de privaciones igualmente distribuidas, se conserva ante cambios incrementales en los ingresos futuros.

La clase de medidas de vulnerabilidad individual en (1.12) es bien general y depende de la especificación de la función $f\left(R\left(z, y_{t}\right)-y_{t+1}^{s}\right)$. Una posible especificación de esta

\footnotetext{
${ }^{14}$ Este axioma se fundamenta en el hecho de que la privación será menor a mayor ingreso futuro esperado.
} 
función, propuesta por los autores es: $f\left(R\left(z, y_{t}\right)-y_{t+1}^{s}\right)=\left(A z^{\alpha} y_{t}^{1-\alpha}-y_{t+1}\right)^{\gamma}$, donde $\gamma>1$. Con lo cual el índice de la expresión (1.12) se transforma en:

$$
V(L)=\sum_{s=1}^{n} p_{s}\left(A z^{1-\alpha} y_{t}^{\alpha}-y_{t+1}\right)^{\gamma}, \gamma>1
$$

Tal especificación sigue siendo no obstante bien general. Para $\alpha=0$, el indicador toma la forma de un índice de pobreza esperada tipo FGT (Foster et al., 1984), donde el umbral de referencia de la vulnerabilidad a la pobreza es la misma línea de pobreza. En el extremo contrario, para $\alpha=1$, el indicador se transforma en el índice de Povel (2010), midiendo así, la vulnerabilidad relativa vinculada al riesgo de obtener caídas en el bienestar futuro con respecto al nivel de bienestar actual. ${ }^{15}$

El criterio de identificación de la vulnerabilidad a la pobreza que se deriva de este enfoque, tiene también un carácter bien general. Tal criterio de identificación podría definirse como sigue:

\section{Criterio 5 (Vulnerabilidad como riesgo de caer bajo un umbral de bienes-} tar relativo asociado a la pobreza): Sea $z$, la línea de pobreza; sean $y_{t}$ e $y_{t+1}$ los resultados del bienestar de un individuo cualquiera en los períodos $t$ y $t+1$ respectivamente; sea $R\left(z, y_{t}\right)$ una media ponderada entre $z$ e $y_{t+1}$. Entonces, un individuo se define vulnerable a la pobreza si y solo si, enfrenta una lotería de privación: $L=p_{1}, R\left(z, y_{t}\right)-$ $y_{t+1}^{1} ; \ldots ; p_{n}, R\left(z, y_{t}\right)-y_{t+1}^{n}$, con probabilidades $p_{1}, \ldots, p_{n}$, tal que, $R\left(z, y_{t}\right)-y_{t+1}^{s} \geq 0, \forall s$, y tal que, existen algunos estados de naturaleza de la lotería en cuestión para los que se cumple que: $y_{t+1}^{s} \leq z$ y $p_{s}>0$.

\section{Análisis crítico}

El planteamiento híbrido que se define en este enfoque, levanta varios temas nuevos para la discusión del concepto de vulnerabilidad a la pobreza. Por un lado, la propuesta destaca la importancia del nivel inicial de bienestar como variable proxi de la capacidad para el manejo de riesgos. Ello concuerda plenamente con la evidencia empírica de estudios anteriores, respecto de que los hogares de menores ingresos tienen menor capacidad para suavizar su consumo a través del tiempo. Pero además de ello, se indica que el riesgo de obtener pérdidas de bienestar tiene características individuales y que no importa solamente

\footnotetext{
${ }^{15}$ Los representantes de este enfoque al parecer se inclinan por un valor intermedio: $0<\alpha<1$.
} 
si el individuo cae o no bajo la línea de pobreza. También importa desde que nivel de bienestar inicial cae. Es decir, se indica que la vulnerabilidad debe ser mayor, cuanto mayor sea la pérdida de bienestar asociada a la caída.

Un punto fuerte de este enfoque es enfatizar en la naturaleza asimétrica de la variación de bienestar vinculada a la vulnerabilidad. Este es un señalamiento que ya se conocía antes en la literatura (Calvo, 2005 y 2007; Povel, 2010). Pero de todas maneras, el trabajo de Dutta et al. (2011) viene a reforzar la argumentación en esta dirección. Se recalca otra vez, que no todas las desviaciones respecto de un nivel de bienestar son relevantes para evaluar la vulnerabilidad a la pobreza, sino solamente las desviaciones a la baja.

Otras virtudes del enfoque son, su fundamentación axiomática y su ordenamiento de los resultados del bienestar a partir de un criterio comúnmente aceptado, ya que el planteamiento de loterías de privación, se apoya en la teoría de la utilidad esperada de von Neumann y Morgenstern (1944).

Sin embargo, el enfoque tiene también aspectos que dan cabida a fuertes críticas. El más importante de ellos es que, desde esta perspectiva muchos de los pobres crónicos no son vulnerables a la pobreza, como efectivamente deberían serlo. El caso más extremo, es el de un individuo con ingreso inicial cero y que enfrenta una lotería degenerada, en la que todos sus ingresos futuros son cero con certidumbre. Desde nuestro punto de vista este individuo es en extremo vulnerable a la pobreza, ya que se encuentra en total estado de indefensión. No obstante, desde la óptica de Dutta et al. (2011: 750), aunque tales individuos sufren una extrema privación, deben ser considerados solamente pobres y no vulnerables,${ }^{16}$ tal como lo indican en la siguiente elocución que citamos textualmente: "... nos centraremos sólo en las personas que tiene la posibilidad de caer en la pobreza en el futuro. Aquellos individuos cuyos ingresos futuros en todos los estados de naturaleza son cero tampoco son considerados. Estos son los más desfavorecidos, pero ya que la privación máxima es un evento seguro, la vulnerabilidad, que en esencia atañe a la incertidumbre respecto del futuro, no puede ser un tema relevante para estos individuos". ${ }^{17}$

Tal apreciación se debe a que el enfoque se centra en el componente de riesgo de la vulnerabilidad a la pobreza, sin tener en cuenta el elemento de pobreza esperada.

\footnotetext{
${ }^{16}$ Desde la perspectiva de Povel (2010) tal individuo tampoco sería considerado vulnerable.

${ }^{17}$ La traducción corresponde al autor. El texto original en inglés es el siguiente: "...we will be focussing only on individuals who has a chance of falling in to poverty in the future. Individuals whose future income in all states equal zero are also not considered. These are the most deprived, but since maximal deprivation is certain vulnerability, which essentially is about the uncertainty in the future, may not be a relevant issue for such individuals".
} 
Desde nuestra perspectiva en cambio, este segundo elemento también es esencial para la evaluación de la vulnerabilidad a la pobreza. Los vulnerables a tal privación no son solamente aquellos que están en riesgo de obtener pérdidas de bienestar que los lleven bajo la línea de pobreza. También son aquellos que están en riesgo de continuar sumidos en la pobreza, ya sea en forma incierta o con total certidumbre. Tal y como lo señalan Calvo y Dercon (2007b: 8): "Si la vulnerabilidad es acerca de la amenaza de la pobreza, la certidumbre de ser pobre es empero una dominante e irresistible amenaza. El concepto no se limita a aquellos a quienes los vientos puedan soplar hacia la pobreza o fuera de ella. La vulnerabilidad atañe al riesgo, pero no solamente a ello". ${ }^{18}$

Otra limitación de este enfoque es que en la práctica utiliza dos umbrales para la identificación de la vulnerabilidad, lo cual puede inducir a algunas confusiones de interpretación. Un primer umbral es la propia línea de pobreza, pues solo aquellos individuos con probabilidades positivas de obtener caídas de bienestar bajo la línea de pobreza son considerados vulnerables. El segundo umbral es la media ponderada entre la línea de pobreza y el nivel de bienestar inicial. Solamente aquellos individuos con probabilidad de caer bajo este último umbral son considerados vulnerables. Entonces, el problema de identificación se vuelve complejo en ciertos casos. Supongamos como ejemplo el caso de un individuo que posee con certeza un nivel de bienestar inicial igual a $0,5 z$ y que espera recibir con certeza en el próximo período, una mejora de bienestar que lo lleva a $0,76 z$. Desde nuestra perspectiva tal cambio provoca en este individuo una disminución en la intensidad de su vulnerabilidad, más sin embargo, sigue siendo vulnerable a la pobreza en el sentido de que enfrenta la amenaza segura de seguir siendo pobre. Su pobreza esperada es positiva. Sin embargo, de acuerdo con el enfoque de Dutta et al. (2011) este individuo no necesariamente es vulnerable, pues para el siguiente período no tendría necesariamente una caída bajo el segundo umbral de referencia. Por ejemplo, si adoptamos como umbral de referencia un promedio simple entre la línea de pobreza y el nivel inicial de ingresos, este individuo no será vulnerable, ya que no caerá bajo tal umbral, pues: $R\left(z, y_{t}\right)=0,5 z+0,5 y_{t}=0,5 z+0,5(0,5 z)=0,75 z<y_{t+1}$.

Finalmente, los autores no ilustran la manera de calcular el índice de vulnerabilidad que proponen. Dejando abierta así la pregunta no trivial, de como calcular los ingresos

\footnotetext{
${ }^{18} \mathrm{La}$ traducción corresponde al autor. El texto original en inglés es el siguiente: "If vulnerability is about the threat of poverty, certainty of being poor is but a dominant, irresistible threat. The concept is not confined to those whom the winds might blow into poverty or out from it. Vulnerability is about risk, but not only about it.".
} 
futuros y las probabilidades futuras.

\subsection{Enfoques de vulnerabilidad como pobreza espe- rada}

\subsubsection{Vulnerabilidad como probabilidad de pobreza futura}

Esta línea de investigación define la vulnerabilidad a la pobreza como la probabilidad que existe en el período corriente de encontrarse en un estado de pobreza en el futuro. Se trata de un concepto de vulnerabilidad que propone capturar el riesgo de caer bajo el umbral de pobreza $z$, a través de una medida de pobreza esperada. La idea se apoya en el concepto de riesgo como el valor esperado de obtener una caída bajo cierto umbral objetivo, que fue desarrollado por Fishburn (1977).

Este enfoque fue planteado inicialmente por Christiaensen y Boisvert (2000) y Prichett et al. (2000). En orden cronológico, le siguieron luego los trabajos de Chaudhuri et al. (2002), Chaudhuri y Christiaensen (2002), Kamanou y Morduch (2002), Chaudhuri (2003), Suryahadi y Sumarto (2003) y Christiansen y Subbarao (2005). ${ }^{19}$ Se trata de uno de los enfoques de vulnerabilidad a la pobreza que más se ha popularizado, tanto en el ámbito de las publicaciones académicas, como en la documentación metodológica del Banco Mundial (véase por ejemplo, el capítulo 12 del manual de Haughton y Khandker, 2009).

De acuerdo con este enfoque, la vulnerabilidad a la pobreza del hogar $j$, en el período $t$, se define como el valor esperado del índice de pobreza FGT:

$$
V P E_{j, t}=\int_{0}^{z}\left(\frac{z-c_{i, t+1}}{z}\right)^{\alpha} f\left(c_{j, t+1}\right) d c_{j, t+1}
$$

\footnotetext{
${ }^{19} \mathrm{Al}$ parecer este enfoque surge de un esfuerzo colectivo, ya que los nombres de los investigadores que lo desarrollaron frecuentemente se entrelazan. Por ejemplo, Shubham Chaudhuri aparece como autor principal en varios de los documentos de trabajo seminales del enfoque. Sin embargo, los coautores de Chaudhuri et al. (2002) son Jyotsna Jalan y Asep Suryahadi. La primera, había sido coautora unos años antes, de un destacado artículo sobre pobreza crónica y transitoria (Jalan y Ravallion, 2000). El segundo, es uno de los coautores de Prichett et al. (2000) y luego publica un artículo con Sudarno Sumarto (Suryahadi y Sumarto, 2003). Por su parte, Luc J. Christiaensen, es coautor con Richard N. Boisvert (Christiansen y Boisvert, 2000), pero también con Shubham Chaudhuri (Chaudhuri y Christiaensen, 2002) y luego con Kalanidhi Subbarao (Christiansen y Subbarao, 2005). Además detrás de todos ellos aparece la figura de un maestro experimentado en estas lides, quien ya había escrito antes con varios de estos investigadores. Ese maestro es Martin Ravallion.
} 
Donde $c_{j, t+1}$ es el consumo per cápita o el consumo equivalente del hogar $j$ en el período $t+1, f\left(c_{j, t+1}\right)$ es la función de densidad de probabilidad del consumo del hogar, $z$ es la línea de pobreza y $\alpha$ es el habitual parámetro de aversión a la pobreza de los índices tipo FGT (Foster et al., 1984). Para enfatizar que la vulnerabilidad a la pobreza de un hogar, es su probabilidad de ser pobre $F(z)$, ponderada por la expectativa de la privación relativa de bienestar (medida por el índice FGT), la expresión anterior frecuentemente es expresada también del siguiente modo:

$$
V P E_{j, t}=F(z) \int_{0}^{z}\left(\frac{z-c_{i, t+1}}{z}\right)^{\alpha} \frac{f\left(c_{j, t+1}\right)}{F(z)} d c_{j, t+1}
$$

Cuando $\alpha$, es cero, el índice de vulnerabilidad representa simplemente, la probabilidad de que el consumo del hogar caiga bajo la línea de pobreza en el siguiente período:

$$
V P E_{j, t}=F(z)=\operatorname{Pr}\left(c_{j, t+1} \leq z\right)
$$

Además, como lo señalan Christiaensen y Boisvert (2000), por analogía a los índices convencionales de pobreza, para $\alpha=1$ el índice en (1.15) representa la probabilidad de ser pobre ponderada por la brecha de pobreza esperada. Mientras que para $\alpha=2$ dicho indicador representa la probabilidad de ser pobre, ponderada por la profundidad de la pobreza esperada.

Uno de los motivos por los cuales posiblemente se ha popularizado este enfoque, se debe a la posibilidad de estimar (1.16) con datos de corte transversal (Chaudhuri et al., 2002; Chaudhuri y Christiaensen, 2002). Los datos de panel no están disponibles usualmente para los países en desarrollo, y por ello, disponer de una medida de vulnerabilidad que pueda estimarse con datos de corte transversal resulta muy atractivo. Para ello se incorporan algunos supuestos fuertemente simplificadores. En primer lugar, se requiere suponer algún tipo de distribución de probabilidad del consumo de los hogares, para lo cual se elige usualmente la distribución log-normal. Por otra parte, dado que en este enfoque se estima una probabilidad futura utilizando datos del período anterior, entonces, hay que suponer que la distribución de probabilidad del período corriente y sus parámetros, se mantienen invariantes en el futuro. Es decir, se supone que el consumo de los hogares es una variable aleatoria que sigue un proceso estacionario fuerte. Finalmente, dados estos supuestos, es posible estimar la probabilidad indicada en (1.16), a partir de estimaciones 
del valor esperado del consumo y de su varianza en el período actual. Con datos de corte transversal, tales estimaciones se hacen condicionando el consumo a un set de variables explicativas y suponiendo errores heterocedásticos.

Al considerar los supuestos anteriores, la expresión (1.16) se transforma en:

$$
V_{j t}=\int_{0}^{\ln z} f\left(\ln c_{j, t+1} \mid X_{j t}\right) d c_{j, t+1}=\Phi\left(\frac{\ln z-E\left(\ln c_{j, t+1} \mid X_{j t}\right)}{\sigma_{c_{j, t+1} \mid X_{j t}}}\right)
$$

Donde, $f\left(\ln c_{j, t+1} \mid X_{j t}\right)$ es la función de densidad del logaritmo neperiano del consumo del hogar $j$ (que se supone gaussiana), en el período $t+1$ (e igual a la del período $t$ ), condicional a un set de características observables, denotadas como $X_{j t}$. Mientras que $E\left(\ln c_{j, t+1} \mid X_{j t}\right)$ y $\sigma_{c_{j, t+1} \mid X_{j t}}$, son la esperanza condicional y la desviación estándar condicional de dicha variable aleatoria, respectivamente.

Adicionalmente, en el enfoque se determina un umbral de probabilidad para identificar a los hogares cuya vulnerabilidad es relevante. Tal umbral de probabilidad usualmente es 0.5. ${ }^{20}$ Así, un hogar se considera vulnerable en un sentido relevante, si su probabilidad de ser pobre es igual o mayor a tal umbral.

Una variante al índice definido en (1.16), considerando un horizonte temporal con múltiples períodos hacia el futuro, es la propuesta de Prichett et al.(2000). De acuerdo con tal planteamiento, la vulnerabilidad del hogar $j$, se mide por la probabilidad de observar al menos un episodio de pobreza en algún período futuro, lo cual es igual a uno, menos la probabilidad de no ser pobre en ningún período futuro. Ello se expresa del siguiente modo:

$$
R_{j, t}(n, z)=1-\left[\left\{1-\operatorname{Pr}\left(c_{j, t+1} \leq z\right), \ldots, 1-\operatorname{Pr}\left(c_{j, t+n} z\right)\right\}\right]
$$

Donde $R_{j, t}(n, z)$ denota el riesgo de caer en un estado con nivel de bienestar igual o inferior a $z$, en alguno de los $n$ períodos futuros. Considerando (1.18), Prichett et al.(2000) definen que un agente es vulnerable, cuando $R_{j, t}(n, z)$ es mayor que cierto umbral de probabilidad $p$, tal y como se establece en la siguiente condición:

$$
V P E_{j, t}(n, z, p)=I\left[R_{j, t}(n, z)>p\right]
$$

En donde $I\left[R_{i, t}(n, z)>p\right]=1$, si se cumple que $R_{i, t}(n, z)>p$, y es igual en cualquier

\footnotetext{
${ }^{20}$ Otro umbral utilizado al implementar el enfoque de Chaudhuri, es la proporción de pobres dentro de la población.
} 
otro caso.

En resumen, el criterio general para identificar a un hogar como vulnerable a la pobreza, de acuerdo con este enfoque, puede enunciarse como sigue:

Criterio 6 (Vulnerabilidad como probabilidad de pobreza futura): Un hogar j, es vulnerable a la pobreza, en el período t, si y sólo si, su probabilidad de ser pobre en el futuro es mayor o igual que cero. La vulnerabilidad a la pobreza se considera relevante, si tal probabilidad es mayor o igual a cierto umbral de probabilidad $p$.

Por otra parte, si asumimos la definición de vulnerabilidad enunciada en (1.17), que proviene de Chaudhuri et al. (2002) y si tomamos como umbral de vulnerabilidad la probabilidad de 0.5 , la condición relevante para determinar si un hogar sufre vulnerabilidad a la pobreza vendría a ser:

$$
\Phi\left(\frac{\ln z-E\left(\ln c_{j, t+1} \mid X_{j t}\right)}{\sigma_{c_{j, t+1} \mid X_{j t}}}\right) \geq 0,5
$$

Teniendo en cuenta además que en una distribución normal, la esperanza matemática acumula una probabilidad de 0.5, entonces, la condición anterior es equivalente a:

$$
\int_{0}^{\ln z} f\left(\ln c_{j, t+1} \mid X_{i t}\right) d c_{j, t+1} \geq \int_{0}^{E\left(\ln c_{i t} \mid X_{i t}\right)} f\left(\ln c_{j, t+1} \mid X_{i t}\right) d c_{j, t+1}
$$

De lo cual se deduce el siguiente criterio de identificación de la vulnerabilidad como pobreza esperada.

Criterio 6a (Chaudhuri-Vulnerabilidad como pobreza esperada): Un hogar $j$, es vulnerable a la pobreza en el período $t$, si y sólo si $E\left(\ln c_{j, t+1} \mid X_{j t}\right) \leq \ln z$.

\section{Análisis crítico}

A pesar de que este enfoque ha sido uno de los más populares, ha sido también posiblemente el más criticado en la literatura (Hoddinott y Quisumbing, 2003; Ligon y Schechter, 2003; Calvo y Dercon, 2005 y 2007b; Povel, 2009; Dutta et al., 2011; Klasen y Povel, 2013).

Algunas de sus limitaciones saltan a la vista, como el hecho de apoyarse en fuertes 
supuestos para predecir una probabilidad futura. En realidad no sabemos si las distribuciones de probabilidad del consumo de cada uno de los hogares son log-normales. Tampoco sabemos si tales distribuciones son iguales para todos los hogares, o si tales distribuciones permanecen invariantes en el tiempo. La estacionariedad fuerte es muy cuestionable para las series de consumo o ingreso de los hogares individuales. Incluso, la estacionariedad débil puede ser cuestionable en algunos aspectos. Por ejemplo, en un horizonte temporal de unos pocos años, el valor esperado del consumo puede no ser estacionario, al estar afectado por movimientos de tendencia de distinta índole. Debidos por ejemplo a cambios en el ciclo de vida, acumulación de capital humano, cambios en el ciclo económico, etcétera.

Por otra parte, la estrategia de corte transversal para la estimación de la varianza propuesta por Chaudhuri et al. (2002) solo tendría sentido si el objetivo fuese estimar la volatilidad intra-grupos condicional a un set de características. En cambio, para estimar la varianza de los resultados del bienestar individual de los hogares, necesariamente se requieren datos en la dimensión temporal. Es decir, ello debe hacerse con información de paneles. Asimismo, los datos de corte transversal tampoco permiten controlar posibles sesgos de endogeneidad debido a la influencia de co-variables inobservables que afectan la media y la varianza.

Otra limitación reconocida de este enfoque, es su insensibilidad al riesgo como variabilidad. Aspecto que fue señalado ya en Ligon y Schechter (2002) y en Hoddinott y Quisumbing (2003). Ello tiene que ver con que dos hogares con igual pobreza esperada, pueden tener distinta variabilidad en los resultados del bienestar y por lo tanto distinto riesgo. Es más, siguiendo este enfoque de vulnerabilidad uno podría incluso reducir perversamente la probabilidad de ser pobre de un hogar, asignándole más riesgo. Hoddinott y Quisumbing (2003: 15-16), proponen el siguiente ejemplo ilustrativo de este problema: supongamos un escenario en el que un hogar se encuentra marginalmente bajo la línea de pobreza con certidumbre. En un segundo escenario le incorporamos al mismo hogar una dispersión de resultados, tal que, el hogar conserva el valor esperado de su bienestar, pero ahora el $50 \%$ de los resultados esperados se encuentran por encima de la línea de pobreza y el otro $50 \%$ se encuentra por debajo de ese umbral. Nótese que si el hogar es adverso al riesgo, en el segundo escenario se encuentra en peor situación. Sin embargo, su probabilidad de ser pobre se ha reducido de 1 a 0.5, debido a la asignación de riesgo. El responsable de ejecutar las políticas públicas, siguiendo este enfoque, estaría incentivado 
entonces a asignar más riesgo a los hogares para disminuir así su probabilidad de ser pobres.

A continuación, en la figura 1.1 mostramos un ejemplo similar para la mejor comprensión de este punto. Las curvas continua y punteada representan las distribuciones de probabilidad del consumo de dos hogares, mientras que la línea recta de color rojo representa la línea de pobreza. Los datos se encuentran normalizados a la línea de pobreza, por lo que esta última es igual a uno. Vemos que los dos hogares tienen el mismo valor esperado en los resultados de su bienestar. Dicho valor esperado es 0.8 y está representado por la línea azul.

Figura 1.1: Ejemplo hipotético de dos hogares con la misma media y distinta varianza en los resultados del bienestar.

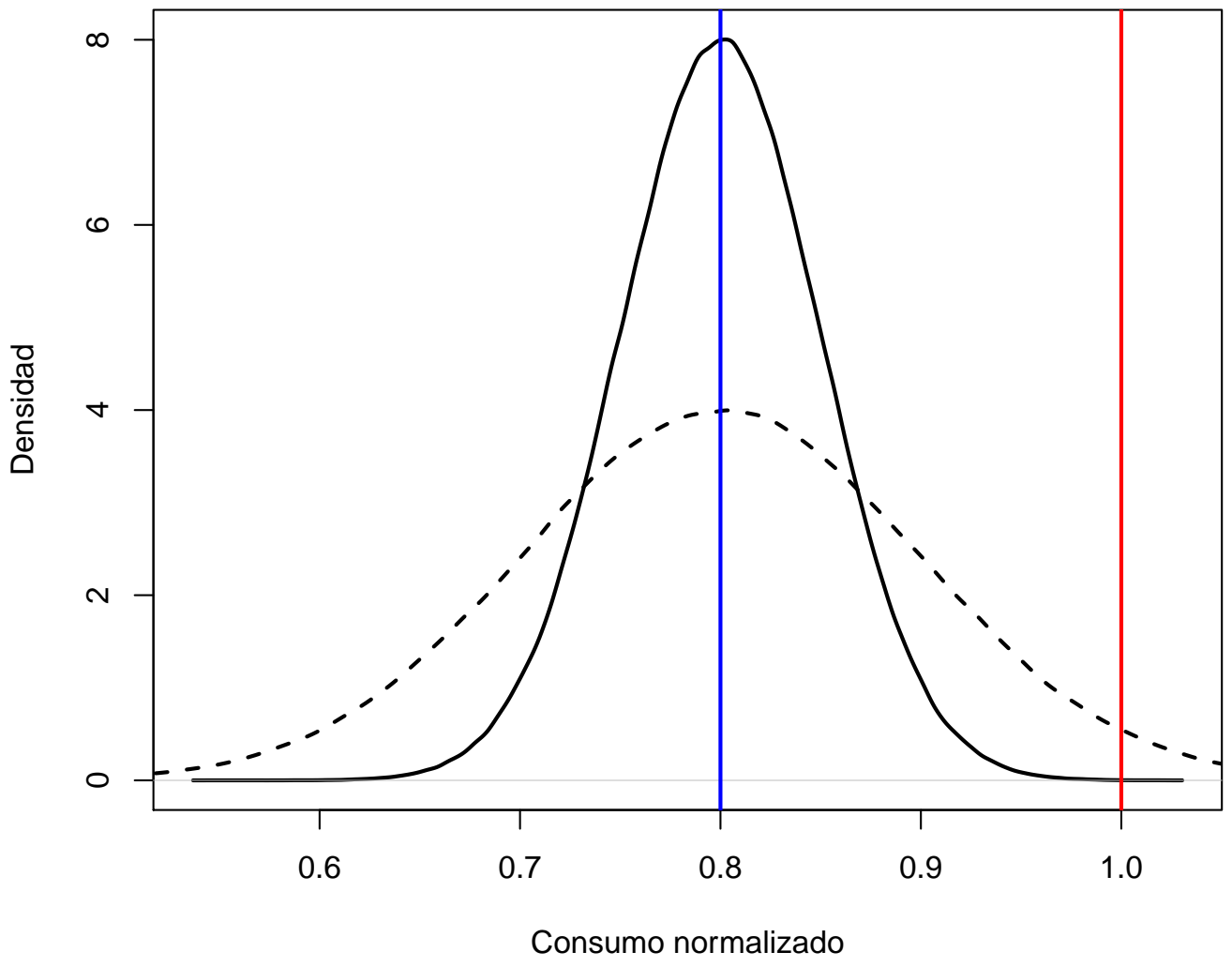

Fuente: Elaboración propia en R.

Notemos sin embargo, que el hogar representado con la línea continua está mejor, ya que disfruta de una menor dispersión (menor varianza) en los resultados de su bienestar. A pesar de ello, bajo el enfoque de vulnerabilidad como probabilidad de ser pobre, este hogar 
sería más vulnerable que el representado en la línea punteada, puesto que su probabilidad de ser pobre es mayor. ${ }^{21}$

Desde nuestra perspectiva, otra limitación importante de este enfoque es su pretensión de identificar la vulnerabilidad con la predicción de la pobreza futura, cuando en realidad solo estima o predice la pobreza presente (con la información presente) condicional a un set de características observables. Con lo cual termina siendo un enfoque de vulnerabilidad como pobreza determinística, a partir de un conjunto de variables observables.

El enfoque reclama proponer un concepto de vulnerabilidad que se distingue del concepto de pobreza, por el hecho ser un fenómeno ex ante, que se enfoca "mirando hacia adelante". En contraposición a la pobreza, que por naturaleza es un concepto ex post, que se construye "mirando hacia atrás". Sin embargo, en la práctica, lo que en realidad se estima en este enfoque, no es el bienestar esperado futuro, sino el actual. Uno puede asumir por supuesto, que tal estimación puede ser usada para predecir los resultados del bienestar futuro. No se puede negar la importancia de la información actual y pasada, para hacer pronósticos respecto del futuro. Pero es muy claro también, que los datos observados en el período corriente son más informativos para la estimación de los resultados del bienestar en el mismo período, que para el pronóstico del futuro.

No obstante lo anterior, a nuestro juicio este enfoque hace una contribución fundamental a la literatura, porque la vulnerabilidad a la pobreza no solamente es riesgo como variabilidad de los resultados del bienestar a la baja. La pobreza esperada posee una naturaleza predecible y por lo tanto refleja claramente una situación de indefensión que a nuestro juicio se condice plenamente con el significado del término "vulnerabilidad". La vulnerabilidad tiene que ver con la incertidumbre, pero no es solamente incertidumbre. La certidumbre de la pobreza profunda es la vulnerabilidad mayor, la más grave y la más difícil de superar debido a que sus causas son estructurales.

\subsubsection{Vulnerabilidad como baja utilidad esperada}

\section{El enfoque}

El enfoque de vulnerabilidad como baja utilidad esperada, es una contribución de Ligon y Schechter (2002 y 2003). La propuesta cobró relevancia en el ámbito académico

\footnotetext{
${ }^{21}$ Casi toda el área bajo la curva continua (su probabilidad) se encuentra antes de la línea de pobreza, es decir, su probabilidad de ser pobre es cercana a uno. Mientras que para el hogar de la línea punteada, el área posterior a la línea de pobreza (su probabilidad de no ser pobre) es mayor.
} 
debido a que establece una comparación de resultados del bienestar fundamentada en la teoría de la utilidad esperada de Von Neumann y Morgenstern (1944) y en el concepto de riesgo de Rothschild y Stiglitz (1970).

Ligon y Schechter (2003) definen la vulnerabilidad como la diferencia entre la utilidad de obtener cierto nivel de consumo bajo certidumbre (por ejemplo la canasta definitoria de la línea de pobreza) y la utilidad esperada del consumo bajo incertidumbre. En términos formales, la vulnerabilidad del hogar o del consumidor $j$ es definida como sigue:

$$
V U E_{j}=U_{j}(z)-E\left[U_{j}\left(c_{j}\right)\right]
$$

Donde $U_{j}($.$) , como es usual, es una función continua y estrictamente creciente (cum-$ ple la condición de no saciedad local), y estrictamente cóncava (cumple la condición de aversión al riesgo), la cual representa las preferencias de cada hogar $j$, en una economía donde se supone que todos los consumidores tienen las mismas preferencias. Al igual que antes, $z$ es la canasta de consumo que define la línea de pobreza, mientras que $c_{j}$ es el consumo per cápita ó equivalente del hogar $j$. E[.] por su parte, es el operador de valor esperado. En el contexto de este marco conceptual, un hogar no se considera vulnerable cuando la utilidad esperada de su consumo es igual o mayor que la utilidad del consumo de $z$ bajo certidumbre.

Uno de los atractivos de este modelo, es que permite descomponer la vulnerabilidad en distintos elementos. Sumando y restando el término $U_{j}\left(E\left[c_{j}\right]\right)$ en la ecuación (1.21), es posible rescribir tal expresión del siguiente modo:

$$
V U E_{j}=\left[U_{j}(z)-U_{j}\left(E\left[c_{j}\right]\right)\right]+\left[U_{j}\left(E\left[c_{j}\right]\right)-E\left[U_{j}\left(c_{j}\right)\right]\right]
$$

De acuerdo con Ligon y Schechter (2003), el primer corchete en (1.23) no contiene ningún tipo de aleatoriedad, porque es simplemente la diferencia entre la utilidad de la pobreza y la utilidad del consumo esperado del agente a través del tiempo. Este componente de la vulnerabilidad es llamado por los autores "componente de pobreza". ${ }^{22}$ Mientras tanto, el término del segundo corchete en (1.23), representa el riesgo que enfrenta el hogar debido a la variabilidad de los resultados de su consumo. Riesgo que es medido en el

\footnotetext{
${ }^{22}$ En un documento de trabajo dado a conocer dos años más tarde, Schechter (2006) vincula este término directamente con la pobreza crónica.
} 
sentido convencional de Rothschild and Stiglitz (1970), por la concavidad de la función de utilidad (dada la desigualdad de Jensen).

La expresión en (1.23) se puede descomponer aún más, para distinguir entre el riesgo idiosincrático (que afecta solamente al agente $j$ ) y el riesgo agregado (que afecta a todos los agentes en cada momento del tiempo). Ello se consigue sumando y restando en la expresión anterior el término $\left[E\left[U_{j}\left(E\left[c_{j} \mid \overline{\mathrm{x}}_{t}\right]\right)\right]\right.$, en donde $\overline{\mathrm{x}}_{t}$ es el centroide o vector promedio de las características observables en el período $t$ :

$$
\begin{aligned}
& V U E_{j}=\left[U_{j}(z)-U_{j}\left(E\left[c_{j}\right]\right)\right] \\
& +\left\{U_{j}\left(E\left[c_{j}\right]\right)-E\left[U_{j}\left(E\left[c_{j} \mid \overline{\mathrm{x}}_{t}\right]\right)\right]\right\} \\
& +\left\{E\left[U_{j}\left(E\left[c_{j} \mid \overline{\mathrm{x}}_{t}\right]\right)\right]-E\left[U_{j}\left(c_{j}\right)\right]\right\}
\end{aligned}
$$

En el primer corchete ahora tenemos el término de pobreza, en el segundo el riesgo idiosincrático y en el tercero el riesgo agregado.

La estimación de este índice de vulnerabilidad requiere además la elección de la forma funcional de $U($.$) . Ligon y Schechter (2003), sugirieron usar una función de utilidad con$ aversión relativa al riesgo constante, conocida como CRRA por sus siglas en inglés: ${ }^{23}$

$$
U\left(c_{j}\right)=\frac{c_{j}^{1-\gamma}}{1-\gamma}
$$

Donde $\gamma$, es un coeficiente constante de aversión al riesgo.

El criterio para identificar a aquellos hogares que son vulnerables a la pobreza según este enfoque conceptual puede ser expresado de la forma que sigue.

Criterio 7 (Vulnerabilidad como baja utilidad esperada): El hogar $j$ es vulnerable a la pobreza si y solo si $E\left[U_{j}\left(c_{j}\right)\right] \leq U_{j}(z)$.

\section{Análisis crítico}

Este criterio de identificación claramente se enfoca en la línea de investigación de la vulnerabilidad como pobreza esperada. Como lo señalan Dutta et al. (2011: 745), el índice de vulnerabilidad que proponen Ligon y Schechter (2003) no es otra cosa que la brecha de pobreza esperada, medida en útiles.

\footnotetext{
${ }^{23}$ Constant Relative Risk Aversion.
} 
Nótese que a diferencia del enfoque de vulnerabilidad como probabilidad de ser pobre, el indicador propuesto en (1.22) si es sensible al riesgo, en virtud de la concavidad de la función de utilidad. Ello en su momento representó un avance importante para el estudio de la vulnerabilidad, puesto que se trata de un enfoque que incorpora el elemento de riesgo como variabilidad, dentro de la medida de pobreza esperada, superando así una limitación importante del enfoque que le antecedió en esta línea de investigación.

Sin embargo, tal medida del riesgo, para los efectos de medición de la vulnerabilidad a la pobreza, ha sido fuertemente criticada en la literatura posterior. Ello se debe a que sostiene una visión simétrica de los efectos de la variabilidad sobre el bienestar de los hogares. En la literatura más reciente (Calvo, 2007b; Povel, 2010; Dutta et al., 2011; Klasen y Povel, 2013; Gallardo, 2013) se enfatiza en que el riesgo relevante para la evaluación de la vulnerabilidad es esencialmente de naturaleza asimétrica. Aquellas desviaciones en la dirección de mejoras del bienestar no determinan la vulnerabilidad, puesto que este fenómeno concierne esencialmente al riesgo de caer o de permanecer bajo cierto umbral de referencia, o bajo el mismo valor esperado del bienestar. ${ }^{24}$

El componente de riesgo de la vulnerabilidad a la pobreza sólo está vinculado con la incapacidad de enfrentar shocks negativos (a la baja) y no con las desviaciones favorables (al alza). Aquellos resultados de incrementos en el bienestar por encima ya sea del umbral de referencia (Calvo, 2005 y 2007; Povel, 2009; Dutta et al. 2011) o ya sea por encima del valor esperado (Gallardo, 2013) no constituyen amenaza alguna para hundirse en la pobreza, ni para permanecer en ella. Tales estados de naturaleza favorables no son escenarios creadores de indefensión o desprotección. No son amenazantes, en el sentido de Calvo y Dercon (2007b: 5). Es más, tales estados de naturaleza tampoco amortiguan o protegen de las posibles caídas en el bienestar. ${ }^{25}$ Por lo tanto, tales desviaciones al alza deben ser consideradas irrelevantes para la identificación de la vulnerabilidad a la pobreza.

Otro aspecto cuestionable del enfoque es que el componente de riesgo en (1.23) depende

\footnotetext{
${ }^{24} \mathrm{El}$ componente de riesgo en (1.23) está construido sobre la hipótesis tradicional de que el comportamiento de aversión al riesgo es simétrico. Esta visión tradicional del riesgo ha sido cuestionada duramente en la literatura de finanzas. Ver por ejemplo: Markowitz (1959); Hogan y Warren (1974), Bawa y Linderberg (1977), Harlow y Rao ( 1989), Estrada (2002).

${ }^{25}$ Por ejemplo, si entre los estados de naturaleza de un individuo pobre con riesgo de empobrecerse aún más, se encontrase el estado de baja probabilidad de ganarse el premio mayor de la lotería, eso no lo vuelve en absoluto menos vulnerable. Si bien, tal circunstancia incrementaría levemente la varianza de sus potenciales resultados de bienestar, ello no afectaría en absoluto la dispersión a la baja en su consumo y por lo tanto, tal probabilidad de volverse millonario no lo le brinda protección ex ante frente a la pobreza. Inclusive si el resultado ex post fuese el más afortunado de los resultados probables.
} 
crucialmente de la decisión del investigador acerca de la especificación de la función de utilidad (mayor concavidad de $U($.$) , implica mayor riesgo) y no de la situación particular$ que enfrenta cada hogar, ni de sus preferencias particulares.

Finalmente, para la estimación empírica de $E\left(U\left(c_{j}\right)\right)$, en este enfoque se utilizan los promedios muestrales de la utilidad, sin tener en cuenta posibles cambios de tendencia en dicho valor esperado. Aspecto importante que fue levantado por Calvo y Dercon (2007a) en el contexto de sus críticas a las medidas de pobreza crónica.

\subsubsection{Enfoque de Calvo y Dercon}

Mientras elaboraba su tesis doctoral en la Universidad de Oxford, el peruano César Calvo escribió junto con el profesor Stefan Dercon, tres documentos de trabajo que significaron un aporte importante para el estudio de la vulnerabilidad a la pobreza (Calvo y Dercon, 2005, 2007a y 2007b). El artículo de Calvo (2008), significó luego la extensión del enfoque al ámbito de la vulnerabilidad multidimensional y la incorporación de mejoras importantes en su estrategia de estimación empírica.

Según estos autores, la vulnerabilidad es una medida de la magnitud en que un hogar sufre la amenaza de enfrentar episodios de pobreza en el futuro. De acuerdo con esto, definen formalmente la vulnerabilidad a la pobreza del siguiente modo:

$$
V=v(z, \mathbf{p}, \mathbf{y})
$$

Donde $V$, es función de la línea de pobreza $z$, del vector de probabilidades $\mathbf{p}=$ $\left(p_{1}, \ldots, p_{k}\right)$ y del vector de resultados $\mathbf{y}=\left(y_{1}, \ldots, y_{k}\right)$, para $k$ estados contingentes.

Calvo y Dercon (2005 y 2007b) argumentan que la función de vulnerabilidad individual debe cumplir nueve axiomas deseables:

- Foco: los cambios en los resultados contingentes por sobre la línea de pobreza no deben afectar $V=v(z, \mathbf{p}, \mathbf{y})$.

- Simetría: El índice de pobreza es inmutable ante las permutaciones de los estados contingentes.

- Continuidad y diferencialidad: $V=v(z, \mathbf{p}, \mathbf{y})$ es una función continua y doblemente diferenciable. 
- Normalidad: La medida de vulnerabilidad se encuentra definida en el intervalo cerrado cero y uno.

- Invarianza a la escala: $V=v(z, \mathbf{p}, \mathbf{y})$ no varía si la población se replica a otra escala.

- Dependencia en probabilidad: la única diferencia entre un estado contingente y otro, son sus probabilidades y resultados.

- Transferencia en probabilidad: Si se transfieren probabilidades junto con sus respectivos resultados entre los distintos estados, la medida de vulnerabilidad no se altera.

- Sensibilidad al riesgo: La vulnerabilidad es mayor con resultados variables para los distintos estados, que con un resultado único promedio para todos los estados.

- Sensibilidad relativa al riesgo constante: un incremento constantemente proporcional de los resultados en todos los estados, no altera la pérdida por riesgo.

Calvo y Dercon (2007b) demuestran que el indicador de vulnerabilidad a la pobreza que satisface los nueve axiomas anteriores, toma la forma del índice de pobreza propuesto por Chakravarty (1983):

$$
V_{(\rho)}=1-E\left[\frac{\operatorname{Min}\left\{y_{j}, z\right\}}{z}\right]^{\rho}, \rho \in(0,1)
$$

Donde $y_{j}$ es el indicador de bienestar del hogar $j$ y $\rho$ es el parámetro de riesgo que cumple con la labor de garantizar la convexidad de la medida de vulnerabilidad.

De acuerdo con lo enunciado en (1.27), el criterio de identificación a la pobreza de Calvo y Dercon (2007b) es el siguiente:

Criterio 8 (Vulnerabilidad como amenaza de ser pobre): El hogar $j$ es vulnerable a la pobreza, si y solo si: $E\left[\frac{\operatorname{Min}\left\{y_{j}, z\right\}}{z}\right]^{\alpha} \leq 1$.

En el trabajo de Calvo (2008) se extiende luego el enfoque hacia la vulnerabilidad a la pobreza multidimensional a partir del siguiente indicador:

$$
V P M_{j}=1-E\left[\left(\sum_{i=1}^{n} \gamma_{i}\left(\frac{\operatorname{Min}\left\{y_{j i}, z_{i}\right\}}{z_{i}}\right)_{i j}^{\rho}\right)^{\frac{\delta}{\rho}}\right]
$$


Donde $V P M_{j}$ es la vulnerabilidad multidimensional a la pobreza del hogar $j, I$ es el número de dimensiones de pobreza, $\gamma_{i}$ es el correspondiente peso relativo de la dimensión de pobreza $i, y_{j i}$ es el resultado del bienestar del hogar $j$ en la dimensión $i, z_{i}$ es el umbral de pobreza en la dimensión $i$, mientras que $\delta$ y $\rho$ son parámetros tales que: $0<\delta<1$ y $0 \leq \rho \leq 1$

\section{Análisis crítico}

La contribución de este enfoque a la literatura, es muy importante. En primer lugar, porque representa el primer intento por construir un índice de vulnerabilidad a la pobreza fundado sobre una base axiomática, ${ }^{26}$ en línea con lo que fueron los fundamentos para la medición de la pobreza desarrollados por Sen (1976 y 1979), Kakwani (1980), Foster et al (1984) y Atkinson (1987). En segundo lugar, estos investigadores fueron los primeros en señalar que las desviaciones del bienestar por encima del umbral de referencia no son relevantes para la evaluación de la vulnerabilidad. Asumen así una perspectiva de riesgo asimétrica, al menos respecto de las desviaciones alrededor de la línea de pobreza. ${ }^{27} \mathrm{Y}$ en tercer lugar, ellos empiezan a usar el término "vulnerabilidad a la pobreza", en lugar del término genérico "vulnerabilidad". Tal novedad no es meramente semántica, sino que constituye una distinción entre el tipo de vulnerabilidad como riesgo por la variabilidad de los resultados del bienestar, en favor de un tipo de riesgo más específico: el de encontrarse amenazado o en peligro de caer en la pobreza o de permanecer en ella.

No obstante lo anterior, el enfoque sigue siendo presa de una concepción simétrica del riesgo, dada por la convexidad del indicador de vulnerabilidad, cuya elección nuevamente depende del criterio del investigador.

\subsubsection{La Propuesta de Chiwaula-Witt-Waibel}

La propuesta de Chiwaula et al. (2011), es una extensión del enfoque de vulnerabilidad como probabilidad de ser pobre, pero que se diferencia de tal perspectiva, porque no requiere del supuesto de una función de distribución de probabilidad, sino que se funda solamente en la estimación de la esperanza y de la varianza del ingreso.

\footnotetext{
${ }^{26}$ Esta propuesta antecede en el tiempo a la de Dutta et al.(2011), que acá hemos presentado antes por su cercanía conceptual con la línea de investigación que enfatiza en el componente de riesgo.

${ }^{27}$ Para Calvo y Dercon (2005 y 2007b), los estados de naturaleza por encima de la línea de pobreza no tienen efecto en la medida de vulnerabilidad. No obstante, el componente de riesgo de su indicador sigue manteniendo simetría, al estar determinado por la convexidad del índice de Chakravarty (1983).
} 
De acuerdo con este enfoque, la probabilidad de ser pobre de un hogar se puede calcular tomando su brecha de pobreza como proporción del intervalo $\left[E\left(y_{j}\right)+\sigma_{y_{j}}, E\left(y_{j}\right)-\sigma_{y_{j}}\right]$, donde $E\left(y_{j}\right)$ es el valor esperado del ingreso del hogar, mientras que $\sigma_{y_{j}}$ es su desviación estándar.

A partir de ello, los autores proponen el siguiente índice de vulnerabilidad a la pobreza:

$$
V_{j}=\operatorname{Pr}\left(y_{j} \leq z\right)=\left\{\begin{array}{cc}
0 & \text { si : }\left[\widehat{E}\left(y_{j}\right)-\widehat{\sigma}_{y_{j}}\right] \geq z \\
\frac{z-\left[\widehat{E}\left(y_{j}\right)-\widehat{\sigma}_{y_{j}}\right]}{2 \widehat{\sigma}_{y_{j}}} & \text { si : }\left[\widehat{E}\left(y_{j}\right)-\widehat{\sigma}_{y_{j}}\right]<z<\left[\widehat{E}\left(y_{j}\right)+\widehat{\sigma}_{y_{j}}\right] \\
1 & \text { si : }\left[\widehat{E}\left(y_{j}\right)+\widehat{\sigma}_{y_{j}}\right] \leq z
\end{array}\right.
$$

Donde $z$ es como siempre, la línea de pobreza, mientras que $\widehat{E}\left(y_{j}\right)$ y $\widehat{\sigma}_{y_{j}}$, son respectivamente los estimadores de $E\left(y_{j}\right)$ y $\sigma_{y_{j}}$. Así, el criterio de identificación de la vulnerabilidad a la pobreza definido en este enfoque es:

Criterio 9 (Vulnerabilidad como probabilidad de ser pobre basada en la varianza): El hogar $j$ es vulnerable a la pobreza, si y solo si: $E\left(y_{j}\right)-\sigma_{y_{j}}<z$.

\section{Análisis crítico}

Un aporte de este enfoque es introducir directamente el uso de la desviación estándar como medida resumen del riesgo, alejándose así, del concepto microeconómico de riesgo propuesto antes en la literatura por Ligon y Schechter (2003). De tal modo, se asume una medida de riesgo estadística, comprensible y comúnmente aceptada. Esto representa una contribución importante, puesto que la desviación típica es diferente para cada hogar y depende de sus propias características y condiciones de riesgo. A diferencia de los índices propuestos por Ligon y Schechter (2003) y Calvo y Dercon (2005 y 2007b), en donde el riesgo depende de parámetros predefinidos por el investigador para determinadas formas funcionales (la función de utilidad en el primer caso y el parámetro de riesgo del índice de pobreza de Chakravarti en el segundo).

Sin embargo, el enfoque mantiene una visión simétrica del riesgo, al usar la desviación estándar como parámetro para medirlo. El uso de tal medida de riesgo es cuestionable porque las distribuciones de probabilidad del bienestar de los hogares no son necesariamente simétricas. Dos hogares que poseen el mismo valor esperado y la misma varianza 
en sus ingresos pueden tener distintos niveles de exposición al riesgo a la baja, ya que pueden tener distintas asimetrías en sus correspondientes distribuciones de probabilidad. Este hecho lo ilustramos en el siguiente ejemplo que se presenta en la figura 1.2. Las curvas continua y punteada representan las distribuciones de probabilidad de los resultados del consumo de dos hogares. La línea roja representa la línea de pobreza. En el ejemplo, ambos hogares tienen la misma media de consumo, que se representa con la línea azul. La varianza de los resultados del consumo también es la misma para los dos hogares. Sin embargo, sus distribuciones de probabilidad poseen diferentes asimetrías. El hogar cuya distribución está representada con la curva punteada enfrenta un mayor riesgo a la baja (mayor asimetría izquierda) y por lo tanto se encuentra en peor situación. El indicador de vulnerabilidad a la pobreza de Chiwaula et al. (2011) es sensible a la variabilidad, pero no lo es a la asimetría, por lo tanto no es capaz de distinguir las diferencias de bienestar entre dos hogares como los representados en este ejemplo.

El concepto de riesgo ha evolucionado mucho en la literatura de finanzas en la dirección de considerar relevante no solo la dispersión de resultados, sino también la asimetría. El uso de la varianza como medida de riesgo ha sido cuestionado desde Markowitz (1959) y luego en el contexto del desarrollo del CAPM asimétrico (Hogan y Warren, 1974; Bawa y Linderberg, 1977; Harlow y Rao 1989; Estrada, 2002). El principal argumento en tales cuestionamientos es que los agentes en general no tienen un comportamiento de aversión a la variabilidad per se, sino solamente de aversión a la dispersión a la baja.

Otras medidas de riesgo que son sensibles a la asimetría, tales como, la semi-desviación típica de la media, la semi-desviación típica de un objetivo y el valor en riesgo, han sido propuestas en la teoría de portafolios, (para un resumen, véase: Roman y Mitra, 2009) para tratar de capturar tal comportamiento de aversión a la dispersión de resultados a la baja.

Desde nuestra perspectiva, cualquier medida de vulnerabilidad debe ser sensible no solamente a la variabilidad de resultados, sino más precisamente a la variabilidad a la baja. Es decir, debe incorporar un concepto de riesgo de naturaleza asimétrica en la dirección de pérdidas de bienestar. 
Figura 1.2: Ejemplo hipotético de dos hogares con la misma media y varianza, pero con distinta asimetría en los resultados del bienestar.

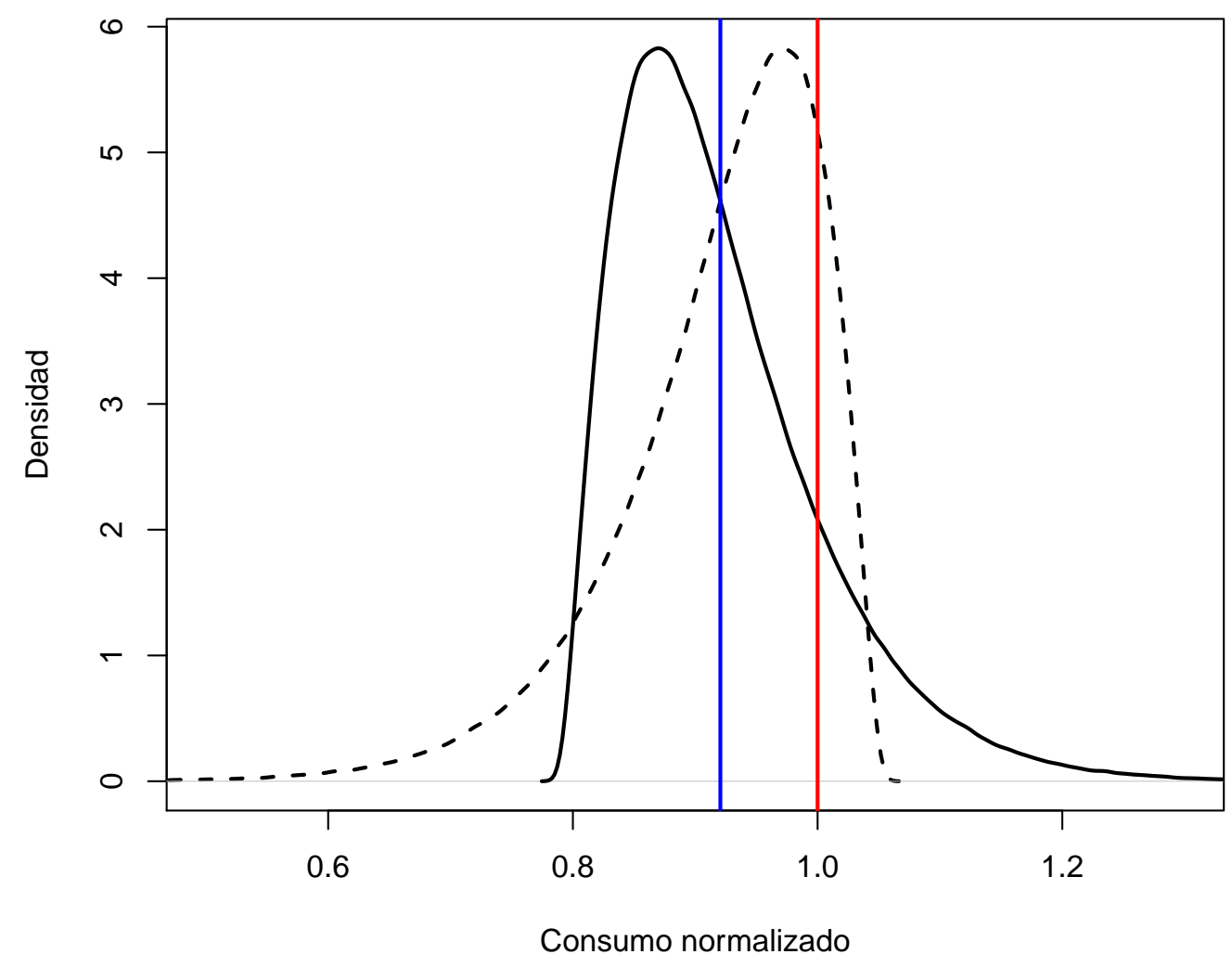

Fuente: Elaboración propia en R.

\subsection{En busca de una síntesis conceptual}

\subsubsection{Vulnerabilidad y vulnerabilidad a la pobreza}

Es fácil perderse en el mar de visiones sobre vulnerabilidad que hemos presentado hasta acá. Como lo señalan Klasen y Povel (2013: 22), citando a Cannon (2008), la vulnerabilidad "se ha convertido en uno de esos términos resbaladizos (como la 'sostenibilidad') que ahora se usan para significar tantas cosas diferentes tal que se encuentran en peligro de perder cualquier significado real". El adjetivo "vulnerable" y el sustantivo "vulnerabilidad" se utilizan ampliamente en distintas áreas del conocimiento. Por ejemplo, en medicina (Flaskerud y Winslow, 1998; Delor y Hubert, 2000; ), prevención de riesgos de desastres naturales (Wisner, 1993; Blaikie et al, 1994; Weichselgartner, 2001; Levine, 2004; Alexander, 2012), criminología (Killias y Clerici, 2000; Daniel, 2010), ciencias 
políticas (Thompson, 1975), psicología (Flouri et al., 2010), etcétera. Ser vulnerable (del latín: vulnerabilis) literalmente significa "que puede ser herido o recibir lesión, física o moralmente" (Real Academia Española, 2001).

En un contexto general, la vulnerabilidad puede ser entendida como un estado de indefensión de cierto agente o sistema (persona, hogar, economía, sistema financiero, sistema climático, etcétera), el cual no posee la capacidad de hacer frente a ciertas amenazas de sufrir un daño, el que eventualmente puede afectar su funcionamiento, su desempeño o su propia existencia. Tales estados de indefensión no son deseados por los agentes o sistemas que los sufren. Pero están determinados por ciertos atributos inherentes al agente o sistema mismo, o al ambiente en el cual se encuentran insertos. Además son estados que ocasionalmente pueden ser provocados por las acciones previas indebidas de los propios agentes que los sufren.

Desde una perspectiva general, la vulnerabilidad de un agente o sistema se puede caracterizar por tres elementos básicos: (i) un daño latente, hacia el cual, el agente o sistema vulnerable se encuentra expuesto; (ii) la existencia de un conjunto de condiciones (debilidades) inherentes al agente o sistema, tales que lo sitúan en un estado de indefensión o desprotección; y (iii) la posible presencia de shocks amenazantes, ante los cuales el daño latente puede ser gatillado o agravado.

El primer elemento es definitorio del tipo de vulnerabilidad a la que hacemos referencia. No tiene mucho sentido usar el concepto de vulnerabilidad en forma vaga, sin aclarar frente a que tipo de daño el agente o sistema en cuestión se encuentra en estado de indefensión. En el contexto médico por ejemplo, un enfermo de VIH que no está protegido por la terapia retroviral, es vulnerable en el sentido de que puede ser afectado por el daño de las enfermedades oportunistas. Mientras que en el contexto de la prevención de riesgos ambientales, las comunidades vulnerables son aquellas expuestas a sufrir daños (destrucción, muerte) provocados por desastres naturales. En el contexto de los enfoques que fueron tratados en la sección anterior, la vulnerabilidad también está ligada siempre a cierto daño. El daño de desviarse del nivel de bienestar promedio (Glewwe y Hall, 1999; Dercon y Krishnan, 2000; Amin el at., 2003; etcétera), o el daño de sufrir una caída en el bienestar actual (Povel, 2009; Dutta et al., 2011), o el daño de padecer pobreza (Chauduri et al., 2002; Calvo y Dercon, 2007b; Chiwaula et al., 2011; etcétera), o el daño de caer bajo cierto equivalente cierto que puede ser también la línea de pobreza (Ligon y Schechter, 
2003).

El segundo elemento de la vulnerabilidad tiene que ver con ciertas debilidades o incapacidades del agente o sistema, las cuales lo sitúan en una situación de desprotección. Estas debilidades son la causa misma de la vulnerabilidad, en el sentido que debido a ellas, el agente se encuentra ya sea ante un resultado casi seguro de sufrir el daño, o frente a un resultado incierto, en dependencia de la ocurrencia de ciertos shocks que el agente no tiene capacidad de enfrentar.

Finalmente, el estado de vulnerabilidad se relaciona con la posibilidad de ocurrencia de eventuales shocks, que pueden provocar el daño cuando el agente no lo sufre, o que pueden agravarlo cuando ya lo sufre.

En el fenómeno de la vulnerabilidad a la pobreza están presentes también estos tres elementos. El daño latente es la pobreza. Las debilidades que condicionan el estado de indefensión frente a la pobreza están dadas en primer lugar por un bajo nivel de bienestar esperado (o por sus condicionantes) y en segundo lugar por la incapacidad de enfrentar ciertos shocks adversos. En tal sentido, los vulnerables a la pobreza son aquellos para los cuales la pobreza es un resultado esperado, o los que no siendo pobres en un sentido esperado, tienen un bajo nivel de bienestar que los sitúa en riesgo de caer en la pobreza, debido a su incapacidad de absorber shocks adversos. El tercer elemento esta dado por la potencial presencia de tales shocks en el ambiente de la incertidumbre.

Entre los enfoques analizados en la sección anterior no todos conciernen precisamente al fenómeno de la vulnerabilidad a la pobreza. Algunos de ellos tratan sobre vulnerabilidades vinculadas con otros tipos de daños. ${ }^{28}$ Aunque pueden asociarse con la vulnerabilidad a la pobreza, en el sentido de que la desprotección frente a shocks que afectan el bienestar, puede provocar pobreza o agravarla.

\subsubsection{Elementos claves de la vulnerabilidad a la pobreza}

En el debate presentado en la sección anterior existen muchas divergencias, pero también ha habido progresos muy relevantes y fuertes coincidencias que se deben destacar para intentar acotar el debate. Entre tales elementos cabe indicar los que a continuación se señalan.

\footnotetext{
${ }^{28}$ Ellos son: la propuesta de Povel (2010) y el enfoque de vulnerabilidad como exposición no asegurada frente al riesgo.
} 


\section{Concepto vinculado al riesgo de caer en pobreza o de permanecer en ella}

Intuitivamente, uno puede indicar, tal como se ha hecho otras veces en la literatura (Chaudhuri et al., 2002: 4), que 'los vulnerables a la pobreza son aquellos que, no siendo pobres, se encuentran en riesgo de caer en la pobreza; o que siendo pobres, se encuentran en riesgo de continuar en tal estado de privación'.

Pero teniendo en cuenta las contribuciones de los enfoques analizados, uno debe concluir además que hay dos elementos claves para determinar tal condición de vulnerabilidad. Ellos son: (i) el riesgo de caer en pobreza debido a la variabilidad a la baja de los resultados del bienestar entre los hogares de bajos ingresos y (ii) la pobreza esperada, cuando tal privación de la pobreza es el resultado más probable o predecible. Todos los enfoques analizados han enfatizado ya sea en el primer elemento, ya sea en el segundo, con algunos intentos por considerar una combinación de ambos.

$\mathrm{Al}$ sintetizar ambos elementos uno puede arribar a la siguiente definición:

Definición: Los vulnerables a la pobreza, son aquellos para los cuales se cumple ya sea que el valor esperado de su bienestar es la pobreza, o que no siendo tal dicho valor esperado, se encuentran en riesgo de que las desviaciones por debajo de dicho valor esperado los conduzcan a situaciones de pobreza.

\section{Necesidad de un modelo de incertidumbre}

La vulnerabilidad a la pobreza es un fenómeno que concierne a la incertidumbre y que por lo tanto debe ser abordado a partir del ordenamiento de los resultados del bienestar bajo incertidumbre. Tal ordenamiento de los resultados del bienestar debe fundamentarse necesariamente en algún modelo aceptado para tal fin, tales como: la dominancia estocástica (Whitmore y Findlay, 1978; Levy, 1992), la dominancia en media riesgo (Markowitz, 1987), o la teoría de la utilidad esperada (Von Neumann, y Morgenstern, 1944).

Chaudhuri et al. (2002) aciertan al afirmar, que la pobreza es un fenómeno que uno puede efectivamente observar con la información disponible, mientras que la vulnerabilidad a la pobreza no es observable directamente Ésta solamente se puede estimar. Ello se debe a que la vulnerabilidad está relacionada con la naturaleza estocástica del proceso generador de los resultados del bienestar. De modo que al evaluar la vulnerabilidad, los resultados efectivos solamente nos sirven para estimar el proceso que los generó. A este 
respecto se ha insistido tenazmente en que la vulnerabilidad a la pobreza es una situación ex ante, en contraposición con la pobreza efectiva, la cual concierne a una situación ex post.

No obstante, se hace necesario acá insistir en un punto que puede inducir a confusión: la naturaleza estocástica del proceso generador de los resultados del bienestar no concierne exclusivamente a la predicción del futuro. Sería un error asumir que los eventos pasados (vistos ex post) son sucesos seguros y que solamente los posibles eventos futuros (vistos ex ante) son inciertos. Puesto que los resultados efectivos del pasado, no son más que una de las posibles realizaciones del proceso estocástico que los generó.

La naturaleza estocástica del proceso no desaparece una vez que constatamos los resultados ocurridos ex-post. Si bien, es cierto por un lado que los procesos estocásticos se nos revelan a través de los resultados efectivos, también es cierto por el otro, que tales resultados efectivos no eliminan la incertidumbre vivida ex ante. Es decir, no transforman al fenómeno probabilístico en uno determístico. Más bien: ¡Los resultados efectivos ex-post continúan siendo parte del proceso estocástico del cual provienen!

Por ello, es innecesario invertir tantos esfuerzos en argumentar que el enfoque de vulnerabilidad a la pobreza es exclusivamente uno que ve "hacia adelante" como pronóstico del futuro, siendo que también es relevante evaluar la exposición al riesgo de ser pobre que enfrentan los hogares en el presente, e inclusive, la exposición al riesgo que enfrentaron en el pasado. En este sentido, la naturaleza ex ante de la vulnerabilidad a la pobreza corresponde esencialmente a que es un fenómeno que evalúa el bienestar bajo incertidumbre y no exclusivamente uno que evalúa las pérdidas de bienestar que van a ocurrir en el futuro.

\section{Sensibilidad al riesgo a la baja}

La sensibilidad al riesgo es una característica exigida a cualquier medida de vulnerabilidad razonable. Pero a este aspecto ya indicado por Ligon y Schechter (2002) y por Hoddinott y Quisumbing (2003), debe sumarse además la exigencia levantada por enfoques más recientes en la literatura (Calvo y Dercon, 2007b; Povel, 2009; Dutta et al., 2011; Gallardo, 2013), respecto de que el riesgo relevante para la evaluación de la vulnerabilidad es exclusivamente a la baja.

Desde nuestra comprensión además, la dispersión de interés para evaluar el riesgo a la baja, no es la dispersión por debajo de la línea de pobreza, sino la dispersión de los 
resultados del bienestar por debajo de su valor esperado. Por ello, una medida resumen de dispersión a la baja con buenas perspectivas para identificar y medir el riego de caer en la pobreza es precisamente la semi-desviación típica a la baja respecto de la media (Gallardo, 2013).

\subsection{Conclusiones}

En este trabajo se ha presentado y discutido el problema de la identificación de la vulnerabilidad a la pobreza a partir de la literatura vigente hasta el año 2013.

Como resultado de esta investigación se concluye que los dos elementos para identificar a los vulnerables a la pobreza son: tener un valor esperado del bienestar bajo la línea de pobreza o estar en riesgo de caer en la pobreza, debido a las desviaciones del bienestar por debajo de su valor esperado.

Todos los enfoques de vulnerabilidad analizados incorporan uno de estos dos elementos, o ambos, en sus criterios de identificación. De acuerdo con ello, tales enfoques se pueden clasificar en dos vertientes: los que enfatizan en el elemento de exposición al riesgo y los que enfatizan en el elemento de pobreza esperada.

En la literatura sobre vulnerabilidad a la pobreza han florecido además nuevos planteamientos que extienden el terreno de la discusión hacia la vulnerabilidad a la pobreza relativa, la vulnerabilidad subjetiva a la pobreza y la vulnerabilidad a la pobreza multidimensional.

La principales contribuciones de este trabajo a la literatura son: haber brindado un análisis del estado del arte sobre el concepto de vulnerabilidad a la pobreza y haber indicado los elementos claves para acercar las posiciones del debate en favor de ahondar en la comprensión del fenómeno.

\section{Referencias}

Adelman, I., Subbrao, K. and Vashishtha, P. (1985). "Some dynamic aspects of rural poverty in India". Economic and Political Weekly, 20: A1O3-117.

Ahluwalia, M. (1985). "Rural poverty, agricultural production and prices: a re-examination". Agricultural Change and Rural Poverty (ed. J. W. Mellor and G. M. Desai). Baltimore: John Hopkins University Press. 
Alexander, D. (2012). "Models of Social Vulnerability to Disasters". RCCS Annual Review, 4.

Amin, S., A. Rai, G. Topa (2003). "Does Microcredit Reach the Poor and Vulnerable? Evidence from Northern Bangladesh". Journal of Development Economics, 70(1): 59-82.

Atkinson, A. B. (1987). "On the measurement of poverty". Econometrica, 55: 749-764.

Bawa, V., Lindenberg, E. (1977). "Capital market equilibrium in a mean-lower partial moment framework". Journal of Financial Economics, 5: 189-200.

Bane, M. J., Ellwood, D. T. (1986). "Slipping into and out of poverty: the dynamics of spells". Journal of Human Resources, 2(1): 1-23.

Béné, C. (2009). "Are Fishers Poor or Vulnerable? Assessing Economic Vulnerability in Small-Scale Fishing Communities". Journal of Development Studies, 45(6): 911-933.

Blaikie, P., Cannon, T., Davis, I. (1994). At Risk: Natural Hazards, People's Vulnerability and Disasters. Routledge, London.

Cafiero, C., Vakis R. (2006) "Risk and vulnerability considerations in poverty analysis: Recent advances and future directions". World Bank, Social Protection Discussion Paper, No. 0610.

Calvo, C., Dercon, S. (2005). "Measuring Individual Vulnerability". University of Oxford, Department of Economics Discussion Paper Series, No.229.

Calvo, C., Dercon, S. (2007a). "Chronic Poverty and All That: The Measurement of Poverty over Time". Centre for the Study of African Economies Series, WPS/2007-04.

Calvo, C., Dercon, S. (2007b). "Vulnerability to poverty". CSAE Working Paper 200703 .

Calvo, C. (2008). "Vulnerability to multidimensional poverty: Peru, 1998-2002". World Development, 36(6): 1011-1020.

Cannon, T. (2008). "Reducing people's vulnerability to natural hazards-communities and resilience". WIDER Research Papers 2008/34, ONU-WIDER, Helsinski.

Cochrane, J. (1991). "A simple test of consumption insurance". Journal of Political Economy. 99: 957-976.

Chakravarty, S. (1983). "A new index of poverty". Mathematical Social Sciences, 6(3): 307-313.

Chambers, R., Longhurst, R. and Pacey, A. (eds.) (1981). Seasonal Dimensions to Rural Poverty. London: Francis Pinter. 
Chaudhuri, S., Jalan, J., Suryahadi, A. (2002). "Assessing household vulnerability to poverty from cross-sectional data: a methodology and estimates from Indonesia". Department of Economics Discussion Paper Series, 0102-52, Columbia University.

Chaudhuri, S. y Christiaensen, L. (2002). "Assessing Household Vulnerability to Poverty: Illustrative Examples and Methodological Issues". Presentation at the IFPRI-World Bank Conference on Risk and Vulnerability: Estimation and policy applications", September 23-24, 2002, Washington DC.

Chaudhuri, S. (2003). "Assessing vulnerability to poverty: concepts, empirical methods and illustrative examples". Department of Economics, Columbia University.

Chiwaula, L., Rudolf, W. y Waibel, H. (2011). "An Asset-Based An Asset-Based Approach to Vulnerability: The Case of Small-Scale Fishing Areas in Cameroon and Nigeria, Journal of Development Studies, 47:2, 338-353.

Christiaensen, L. y Boisvert, R. (2000). "On Measuring Household Food Vulnerability: Case Evidence From Northern Mali". Cornell University, Department of Applied Economics and Management Working Papers, WP-2000-05.

Christiaensen, L. y Subbarao, K. (2000). "Towards an Understanding of Household Vulnerability in Rural Kenya". Journal of African Economies, 14(4): 520-558.

Daniel, B. (2010). "Concepts of Adversity, Risk, Vulnerability and Resilience: A Discussion in the Context of the 'Child Protection System' ". Social Policy and Society. 9(2): $231-241$.

Delor, F., Hubert, M. (2000). "Revisiting the concept of "vulnerability' ". Social Science and Medicine, 50: 1557-1570.

Dercon, S., Krishnan, P. (2000). "Vulnerability, seasonality and poverty in Ethiopia". Journal of Development Studies, 36(6), 25-53.

Devereux, S. (2001). "Can social safety nets reduce chronic poverty?". Manuscrito no publicado, presentado en el Seminario de Investigacion sobre Pobreza Crónica de la Escuela de Políticas Públicas de Birmingham University.

Dutta, I., Foster, J., Mishra, A. (2011). "On measuring vulnerability to poverty". Social Choice and Welfare, 37:743-761.

Estrada, J. (2002). "Systematic risk in emerging markets: the D-CAPM". Emerging Markets Review, 3, 365-379.

Fishburn, P. (1977). "Mean-risk analysis with risk associated with below-target re- 
turns". American Economic Review, 67(2): 116-126.

Flaskerud, J. and Winslow, B. (1998). "Conceptualising vulnerable populations healthrelated research". Nursing Research, 47(2): 69-78.

Flouri, E., Mavroveli, S. and Tzavidis, N. (2010). "Modeling risks: Effects of area deprivation, family socioeconomic disadvantage and adverse life events on young children's psychopathology". Social Psychiatry and Psychiatric Epidemiology, 45(6): 611-619.

Foster, J., Greer, J., Thorbecke, E. (1984). "A class of decomposable poverty measures". Econometrica, 52: 761-766.

Foster, J., Greer, J., Thorbecke, E. (2010). "The Foster-Greer-Thorbecke (FGT) poverty measures: 25 years later". Journal of Economic Inequality. 8(4): 491-524.

Gaiha, R., Imai, K. (2009). "Measuring Vulnerability and Poverty Estimates for Rural India". Vulnerability in Developing Countries, capítulo 2, United Nation University Press. Editado por: Naudé, W., Santos-Paulinos, A. y McGillivray, M..

Gallardo, M. (2013). "Using the downside mean-semideviation for measuring vulnerability to poverty". Economics Letters, 120(3): 416-418.

Glewwe, P., Hall G. (1998). "Are some groups vulnerable to macroeconomic shocks than others? Hypothesis tests based on panel data from Peru". Journal of Development Economics, 56(1): 181-206.

Gottschalk, P. (1982). "Earnings Mobility: Permanent Change or Transitory Fluctuations?". The Review of Economics and Statistics, 64(3): 450-456.

Grimard, F. (1997). "Household consumption smoothing through ethnic ties: Evidence from Cote D'Ivoire". Journal of Development Economics, 53(2): 391-422.

Harlow, V., Rao, R. (1989). "Asset pricing in a generalized mean-lower partial moment framework: theory and evidence". Journal of Financial and Quantitative Analysis, 24: 285-311.

Haughton, J. y Khandker, S. (2009). Handbook on Poverty and Inequality, World Bank, Washington, D.C..

Hoddinott, J., Quisumbing, A. (2003). "Methods of microeconometric risk and vulnerability assessments". Social Protection Discussion Paper Series 0324, World Bank.

Hogan, W., Warren, J. (1974). "Toward the development of an equilibrium capitalmarket model based on semivariance". Journal of Financial and Quantitative Analysis, 9: 1-11. 
Hoogeveen, J., Tesliuc, E., Vakis, R. y Dercon, S. (2004). "A Guide to the analysis of risk, vulnerability and vulnerable groups". Policy Research Working Paper,World Bank, Washintong D.C..

Jalan, J., Ravallion, M. (1999). " Are the poor less well insured? Evidence on vulnerability to income risk in rural China". Journal of Development Economics, 58(1): 61-81.

Jalan, J., y Ravallion M. (2000). ”Is Transient Poverty Diferent? Evidence for Rural China". Journal of Development Studies, 36: 82-99.

Kakwani, N. (1980). "On a class of poverty measures". Econometrica, 48(2): 437-446.

Kamanou G, Morduch, J. (2002). "Measuring vulnerability to poverty". WIDER discussion paper, 2002/58.

Killias M., Clerici, C. (2000). "Different Measures of Vulnerability in their Relation to Different Dimensions of Fear of Crime". British Journal of Criminology, 40(3): 437-450.

Klasen, S., Povel, H. (2013). "Defining and Measuring Vulnerability: State of the Art and New Proposals". Capítulo 2 del libro: Vulnerability to Poverty: Theory, Measurement and Determinants, with Case Studies from Thailand and Vietnam, Palgrave Macmillan. Editado por: Klasen, S., Waibel, H..

Kochar, A. (1995). "Explaining household vulnerability to idiosyncratic income shocks". The American Economic Review, 85(2): 159-164.

Levine, C. (2004). "The Concept of Vulnerability in Disaster Research". Journal of Traumatic Stress, 17(5): 395-402.

Levy, H. (1992). "Stochastic dominance and expected utility: Survey and analysis". Management Science, 38: 555-593.

Levy, F. (1977). "How Big Is the American Underclass?". Working paper 0090-1, Urban Institute, Washington, D.C..

Ligon, E., Schechter, L. (2002). "Measuring vulnerability". Manuscrito no publicado, University of California-Berkeley.

Ligon, E., Schechter, L. (2004). "Evaluating Different Approaches to Estimating Vulnerability". Social Protection Discussion Paper Series 0410, World Bank.

Lipton, M. (1983). "Poverty, Undernutritiona and Hunger". World Bank Staff Working Paper No. 597, Washington, D.C.: World Bank.

Longhurst, R., Chambers, R. y Swift, J. (1986). "Seasonality and poverty: implications for policy and research". IDS Bulletin, 17: 67-70. 
Markowitz, H., 1959. Portfolio Selection. Wiley, New York.

Markowitz, H. (1987). Mean-Variance Analysis in Portfolio Choice and Capital Markets. Blackwell, Oxford.

Morduch, J. (1984). "Poverty and Vulnerability". The American Economic Review, 84(2): 221-225.

Povel, F. (2010). "Perceived Vulnerability to Downside Risk". University of Goettingen, Courant Research Centre 'Poverty, Equity and Growth in Developing and Transition Countries: Statistical Methods and Empirical Analysis', Discussion Paper No. 43.

Prichett, L., Suryahadi A. y Sumarto, S. (2000). "Quantifying vulnerability to poverty: a proposed measure, applied to Indonesia”. Policy Research Working Paper No. 2437, The World Bank, Washington, D.C..

Ravallion, M. (1988). "Expected poverty under risk-induced welfare variability", Economic Journal, 98(393): 1171-1182.

Ravallion, M. (1996). "Issues in Measuring and Modelling Poverty". Economic Journal, 106(438): 1328-1343.

Ravallion, M., Chaudhuri, S. (1997). "Risk and insurance in village India: Comment". Econometrica, 65(1): 171-184.

Real Academia Española (2011). Diccionario de la Real Academia Española.

Roman, D. y Mitra, G, (2009). "Portfolio selection models: A review and new directions". Wilmott Journal, 1(2): 69-85.

Rothschild, M., Stiglitz,J. E. (1970). "Increasing risk: I. A definition". Journal of Economic Theory, 2: 225-43.

Schechter, L. (2006). "Vulnerability as a Measure of Chronic Poverty". Chronic Poverty Research Centre (CPRC), Manchester, UK, 17.

Sen, A. (1976). Poverty: an ordinal approach to measurement. Econometrica, 44(2), 219-231.

Sen, A. (1979). "Issues in the Measurement of Poverty". Scandinavian Journal of Economics, 81: 285-307.

Skoufias, E., Quisumbing A. (2005). "Consumption insurance and vulnerability to poverty: A synthesis of the evidence from Bangladesh, Ethiopia, Mali, Mexico and Russia". European Journal of Development Research, 17(1): 24-58.

Suryahadi, A., Sumarto, S. (2003). "Poverty and vulnerability in Indonesia before and 
after the economic crisis". Asian Economic Journal, 17:1, 45-64.]

Tesliuc, E., Lindert, K. (2002). "Vulnerability: A quantitative and qualitative assessment". Guatemala Poverty Assessment Program. Washington, D.C.: World Bank.

Thompson, W. (1975). "Regime Vulnerability and the Military Coup". Comparative Politics, 7(4): 459-487.

Townsend R. (1994). "Risk and Insurance in Village India". Econometrica, 62(3):539591.

Von Neumann, J., y Morgenstern, O. (1944). Theory of Games and Economic Behavior, Princeton University Press.

Watts, M., Bolhe, G. (1993). "Hunger, famine and the space of vulnerability". Geojournal, 30(32): 117-125.

Weichselgartner, J. (2001). "Disaster mitigation: the concept of vulnerability revisited". Disaster Prevention and Management, 10(2): 85-95.

Whitmore, G., Findlay, M. (1978). Stochastic Dominance: An Approach to DecisionMaking Under Risk. Heath, Lexington, MA.

Wisner, B. (1993). "Disaster vulnerability: scale, power and daily life". Geojournal, 30(32): 127-140.

World Bank (2001). World Development Report 2000/2001 - Attacking Poverty. New York, Oxford University Press. 


\section{Capítulo 2}

\section{El problema de la medición}

\section{Resumen}

En este capítulo ${ }^{1}$ se introduce un nuevo enfoque de vulnerabilidad a la pobreza, utilizando la semi-desviación típica a la baja como parámetro de riesgo. Los resultados inciertos del bienestar de los hogares son ordenados mediante un criterio de dominancia, en el marco de un modelo de comportamiento de media-riesgo. Tal ordenamiento permite comparar el bienestar de los hogares bajo incertidumbre, con la línea de pobreza, para identificar así a los hogares vulnerables. Se propone un nuevo índice para medir la vulnerabilidad a la pobreza de la población y se muestra su cálculo en una aplicación empírica con datos de panel para el Perú.

\subsection{Introducción}

La incertidumbre ha sido incorporada al análisis de la pobreza, a través del concepto de vulnerabilidad. Diversos enfoques se han propuesto para medir el riesgo que enfrentan los hogares de pocos recursos, de caer en la pobreza o de permanecer en ella por más tiempo. ${ }^{2}$ Sin embargo, aún prevalece un amplio desacuerdo acerca de que es la vulnerabilidad y acerca de como debe medirse.

\footnotetext{
${ }^{1}$ Una versión resumida de este capítulo ya fue publicada en inglés en la revista Economics Letters bajo el título: "Using the downside mean-semideviation for measuring vulnerability to poverty" (Gallardo, 2013). Por consiguiente, los derechos de propiedad sobre este capítulo ya fueron cedidos por el autor a la revista indicada.

${ }^{2}$ Dos excelentes resúmenes sobre los enfoques conceptuales de la vulnerabilidad a la pobreza pueden encontrarse en Hoddinott y Quisumbing (2003) y Klasen y Waibel (2013)
} 
En el capítulo se introduce un nuevo enfoque de vulnerabilidad a la pobreza, que se fundamenta en el modelo de media-riesgo desarrollado en la literatura de finanzas (Markowitz, 1987). El enfoque parte de la ordenación de los resultados inciertos del bienestar de los hogares a partir del criterio de dominancia en media-riesgo. Ello a su vez permite comparar tales resultados inciertos con la línea de pobreza, consiguiendo así, identificar a aquellos hogares que son vulnerables a tal privación.

El enfoque se arraiga fuertemente en la literatura precedente, pero enfatizando en la naturaleza asimétrica que caracteriza al riesgo de caer en pobreza. De ahí que se sostenga el uso de la semi-desviación a la baja como parámetro de riesgo.

Se propone una nueva medida de vulnerabilidad a la pobreza para la población total, cuyo cálculo depende solamente de la estimación de dos escalares para cada observación: la media muestral y la semi-desviación típica a la baja. La medida propuesta sigue un típico diseño FGT (Foster-Greer-Thorbecke, 1984), que permite el cumplimiento de la base axiomática característica para esta clase de índices de pobreza.

Se presenta además un ejercicio empírico para ilustrar el cálculo del nuevo indicador, utilizando un panel de datos de hogares del Perú.

En la sección siguiente se desarrolla el marco conceptual del nuevo enfoque, a continuación se define el nuevo índice de vulnerabilidad a la pobreza, luego se presenta el ejemplo empírico y al final se ofrecen las conclusiones correspondientes.

\subsection{Marco Conceptual}

\subsubsection{Dos tipos de vulnerabilidad a la pobreza}

En el capítulo anterior he sostenido que la vulnerabilidad a la pobreza es un estado de desprotección que corresponde a dos tipos de situaciones: (i) pobreza esperada, ó (ii) riesgo de caer en pobreza, debido a los posibles desvíos en los resultados del bienestar por debajo de su valor esperado.

El primer tipo de situación atañe al caso en el cual, el valor esperado del bienestar se encuentra por debajo del umbral de pobreza. Chaudhuri et al. (2002), Suryahadi y Sumarto (2003) y Christiansen y Subbarao (2005), propusieron capturar este tipo de vulnerabilidad a través del pronóstico de índices de pobreza tipo FGT (Foster-GreerThorbecke, 1984). Calvo y Dercon (2005, 2007) y Calvo (2008) en cambio, consideraron 
hacerlo a través del índice de Chakravarty (1983), cuya forma funcional permite capturar también el componente de riesgo concerniente a la situación (ii), aunque de manera simétrica respecto de la media. Mientras que Ligon y Schechter (2003) propusieron un diseño a partir de la brecha de pobreza esperada, medida en útiles, que también incorpora un componente de riesgo simétrico, a través de la concavidad de la función de utilidad.

En el segundo tipo de situación, aunque el valor esperado del bienestar del hogar está por encima del umbral de pobreza, éste se encuentra en riesgo de sufrir tal privación, debido a los desvíos aleatorios bajo la media. Los estudios pioneros sobre vulnerabilidad (Morduch, 1994; Glewwe y Hall, 1998; Dercon y Krishnan, 2000 y Amin y Rai, 2003) se focalizaron en esta perspectiva. La vulnerabilidad se planteaba en términos del riesgo que enfrentan los hogares de bajos ingresos, de experimentar pérdidas de bienestar, debido a su incapacidad para suavizar el consumo a través del tiempo. Desde nuestra perspectiva no obstante, enfatizamos que el riesgo relevante para este segundo tipo de vulnerabilidad, no atañe a la variabilidad del bienestar per sé, sino solamente a los desvíos bajo el valor esperado, que caen bajo la línea de pobreza.

Es decir, se trata de un riesgo que es esencialmente de naturaleza asimétrica. Primero, porque atañe a la amenaza de obtener un resultado desfavorable, no siendo relevante por lo tanto para su evaluación, la dispersión de resultados al alza (Calvo y Dercon, 2005 y 2007 levantaron este punto). Segundo, porque si las distribuciones de probabilidad de los resultados del bienestar de los hogares no son simétricas, podríamos computar erróneamente el riesgo de obtener un resultado no deseado, usando datos en la dirección de los resultados deseados. Tercero, porque a los agentes económicos no les disgusta la dispersión per sé, sino solamente la dispersión a la baja, tal como se ha indicado ampliamente en la literatura de finanzas, tanto en el contexto de las críticas a la varianza como medida de riesgo, en favor de la semi-varianza (Markowitz, 1959), como en el contexto del desarrollo del CAPM asimétrico, llamado comúnmente D-CAPM (Hogan y Warren, 1974, Bawa y Linderberg, 1977; Harlow y Rao, 1989; Estrada, 2002).

Cabe indicar además que la vulnerabilidad como pobreza esperada, se encuentra cercanamente asociada a la pobreza crónica o estructural, pero en nuestro concepto no es equivalente a esta como algunas veces se ha señalado (por ejemplo: Schechter, 2006). La pobreza esperada atañe a la amenaza de la pobreza, como un resultado previsible ex ante, independientemente de cómo se revele o se haya revelado ex post la incertidumbre. En 
cambio, la pobreza crónica corresponde a las realizaciones efectivas de pobreza (es decirr ex post), persistentes en el tiempo. Del mismo modo, el segundo tipo de vulnerabilidad se encuentra asociado muy cercanamente a la pobreza transitoria (Morduch, 1994), pero se distingue de ésta porque no corresponde a la realización efectiva de algunos estados de pobreza, sino solamente al potencial riesgo de sufrirlos.

\subsubsection{Formalización del enfoque}

Sea $y_{j}$, la variable aleatoria que representa el resultado del bienestar del hogar $j$, tal que $y_{j}$ es además, la variable focal de política económica utilizada para medir la pobreza (el consumo, el ingreso, o algún índice multidimensional). Sea $y_{j} \geq 0$. Nos situamos en un escenario realista, en el que desconocemos la distribución de $y_{j}$, pero podemos estimar razonablemente su valor esperado $\mu_{j}$ y una medida $r_{j}$, que representa el riesgo de que la variable focal se desvíe por debajo de $\mu_{j}$.

Asumimos que los agentes se comportan racionalmente, con preferencias cuasi cóncavas y estrictamente crecientes en $y_{j}$. Bajo incertidumbre, tales preferencias pueden ser representadas razonablemente por una función de utilidad del tipo: $U\left(\mu_{j}, r_{j}\right)$, con derivadas parciales: $\frac{\partial U_{j}}{\partial \mu_{j}}>0, \frac{\partial U_{j}}{\partial r_{j}}<0, \forall j$, respectivamente. Es decir, los hogares se comportan maximizando $\mu_{j}$ y minimizando $r_{j}$.

Dadas tales preferencias, para cualquier par de variables aleatorias: $y_{j}, y_{k}$, se cumple la siguiente relación de dominancia estricta en media-riesgo (donde $\succ$ denota tal relación de prefencias):

$$
y_{j} \succ y_{k} \Leftrightarrow \mu_{j} \geqslant \mu_{k} \wedge r_{j} \leqslant r_{k}
$$

con al menos una de las desigualdades estricta.

Además suponemos que para efectos de política económica, la autoridad política define una valoración social entre media y riesgo a través de un coeficiente de trade-off: $\gamma>0$. Ello permite comparar cualquier par de variables aleatorias: $y_{j}, y_{k}$, por dominancia en media-riesgo (véase: Ogryczak y Ruszynski, 1999: 15), del siguiente modo:

$$
y_{j} \succ y_{k} \Leftrightarrow \mu_{j}-\gamma r_{j}>\mu_{k}-\gamma r_{k}
$$

Por otra parte, es razonable esperar que la autoridad política sea adversa al riesgo $(\gamma>0)$ y que las ganancias en los resultados esperados del bienestar sean a lo menos tan 
preferidas como el evitar las pérdidas por riesgo $(\gamma \leqslant 1)$. Ello permite acotar razonablemente los valores de $\gamma$ en el intervalo $(0,1]$.

Criterio de Identificación: Sea z el umbral de pobreza bajo certidumbre. Entonces, diremos que el hogar $j$ es vulnerable a la pobreza, si y sólo si $\mu_{j}-\gamma r_{j} \leq z$. La vulnerabilidad a la pobreza toma forma de pobreza esperada (vulnerabilidad de primer momento) y se dice severa, si $\mu_{j} \leq z$; toma forma de riesgo de caer en pobreza (vulnerabilidad de segundo momento) y se dice $\underline{\text { moderada, }}$, si $\mu_{j}>z$, pero $\mu_{j}-\gamma r_{j} \leq z$.

Consideramos que el riesgo $r_{j}$, puede ser evaluado razonablemente (aunque no de manera única) a través de la semi-desviación a la baja, la cual se define del siguiente modo:

$$
\sigma_{j}^{-}=\sqrt{E\left\{\operatorname{Min}\left[\left(y_{j}-\mu_{j}\right), 0\right]^{2}\right\}}
$$

Esta medida de riesgo, es ampliamente utilizada en el ámbito de la teoría financiera (por ejemplo en el modelo D-CAPM, véase: Estrada, 2002) y es consistente además con la dominancia estocástica de segundo orden, en el sentido de Ogryczak y Ruszczynski (1999, 2001) para valores de $\gamma$ acotados en el intervalo considerado.

\subsection{Un índice de vulnerabilidad de la población}

Supongamos una población de $N$ hogares, con subíndices: $j=1,2, \ldots, N$, para cada hogar. Buscamos un índice de vulnerabilidad a la pobreza para la población total $V$, que comparta con otras medidas de pobreza, el cumplimiento de la base axiomática desarrollada por Sen (1976, 1979), Kakwani (1980) y Foster-Greer-Thorbecke (1984). En particular, ceteris paribus, exigiremos que tal índice satisfaga los siguientes axiomas de invarianza, dominancia y sub-agrupación:

INVARIANZA:

1. Foco: Los resultados del bienestar de los hogares no vulnerables a la pobreza no deben afectar $V$.

2. Simetría (Anonimidad): Las permutaciones de $\mu_{j}-\gamma r_{j}$ entre los agentes vulnerables 
a la pobreza, no deben generar cambios en $V$.

3. Invarianza a las réplicas: $V$ no debe cambiar ante replicaciones (clonaciones) de toda la población.

4. Invarianza a la escala: Si se multiplican todos los valores $\mu_{j}-\gamma r_{j}$, y la línea de pobreza $z$, por una constante de escala $\lambda>0, V$ no debe cambiar.

5. Continuidad: $V$ debe ser una función continua.

\section{DOMINANCIA:}

6. Monoticidad: Todo lo demás constante, si $\mu_{j}-\gamma r_{j}$ disminuye para cualquier agente vulnerable $j, V$ debe aumentar.

7. Transferencia: Todo lo demás constante, si los hogares $k$ y $j$ son vulnerables a la pobreza y si $j$ domina en media-riesgo a $k$, entonces, cualquier transferencia de recursos, desde $k$ hacia $j$, tal que como resultado de dicha transferencia se incrementa $\mu_{j}-\gamma r_{j}$ y disminuye $\mu_{k}-\gamma r_{k}$, debe provocar un aumento de $V$.

8. Sensibilidad a las Transferencias: Todo lo demás constante, si los hogares $k$ y $j$ son vulnerables a la pobreza y si $j$ domina en media-riesgo a $k$, entonces, ante cualquier transferencia de recursos, desde $k$ hacia $j$, tal que como resultado de dicha transferencia se incrementa $\mu_{j}-\gamma r_{j}$ y disminuye $\mu_{k}-\gamma r_{k}$, el aumento de $V$ debe ser menor, cuanto mayor sea $\mu_{k}-\gamma r_{k}$.

\section{SUB-AGRUPACIÓN:}

9. Descomposición aditiva: Si se particiona la población total en $M$ sub-grupos: $g_{1}, g_{2}, \ldots, g_{I}$, de tamaños: $n_{1}, n_{2}, \ldots, n_{I}$, entonces, la vulnerabilidad agregada debe ser igual a la suma: $V=\sum_{i=1}^{M} \frac{n_{i}}{N} V_{i}$, donde $V_{i}$ es la vulnerabilidad a la pobreza del grupo $i$.

10. Consistencia sub-grupal: Si se particiona la población total en $M$ sub-grupos: $g_{1}, g_{2}, \ldots, g_{I}$, de tamaños: $n_{1}, n_{2}, \ldots, n_{I}$, entonces, todo lo demás constante, cualquier incremento de la vulnerabilidad en un grupo debe generar un incremento de la vulnerabilidad total de la población.

Considérese el siguiente índice para medir la vulnerabilidad a la pobreza de la población total: 


$$
V_{\alpha}=\sum_{j=1}^{N} \frac{1}{N}\left(\frac{z-\left[\mu_{j}-\gamma r_{j}\right]}{z}\right)^{\alpha} I_{\mu_{j}-\gamma r_{j} \leq z}, \alpha \geqslant 0
$$

Donde $I_{\mu_{j}-\gamma r_{j} \leq z}$ es una función indicadora que vale uno si el hogar $j$ satisface la condición de foco $\mu_{j}-\gamma r_{j} \leq z$ y vale cero si no. El diseño FGT en (2.4) garantiza que en dependencia del valor de $\alpha$, el índice propuesto de vulnerabilidad satisfaga los axiomas anteriores, que son característicos de esta clase de índices de pobreza, ${ }^{3}$ en la forma que se indica a continuación.

Proposición: La medida de vulnerabilidad $V_{\alpha}$ definida en (2.4) cumple con los axiomas 1, 2, 3, 4, 9 y 10 definidos anteriormente, para todo $\alpha \geq 0$; cumple el axioma 6 para $\alpha>0$, el axioma 7 para $\alpha>1$ y el axioma 8 para $\alpha>2$; además $V_{\alpha}$ está definida en el intervalo $[0,1]$ y cumple con el axioma 5 para todo $\alpha$ (excepto en el punto $z$, para $\alpha=0$ ).

\section{Demostración:}

1. El cumplimiento del axioma 1 para todo $\alpha$, está garantizado directamente por la función indicadora: $I_{\mu_{j}-\gamma r_{j} \leq z}$, la cual excluye de la medida de vulnerabilidad $V_{\alpha}$ a todos aquellos hogares que no son vulnerables a la pobreza: $I_{\mu_{j}-\gamma r_{j} \leq z}=0 \Leftrightarrow \mu_{j}-\gamma r_{j}>z$.

2. El cumplimiento del axioma 2 para todo $\alpha$ se garantiza trivialmente por la propiedad conmutativa de la suma. Las permutaciones de los elementos $\mu_{j}-\gamma r_{j}$ entre los agentes vulnerables a la pobreza solo cambian el orden de los sumandos en (2.4), pero no alteran el resultado de la suma.

3. Respecto del cumplimiento del axioma 3 para todo $\alpha$, notemos lo siguiente: si replicamos (clonamos) una población de tamaño $N, k$ veces, donde $k \in \aleph, k \geq 2$, como resultado obtendremos una nueva población de tamaño $k N$, donde cada observación se repetirá $k$ veces. Por lo tanto, la nueva medida de vulnerabilidad a la pobreza de la población replicada será:

$$
V_{\alpha}=\sum_{j=1}^{k N} \frac{1}{k N}\left(\frac{z-\left[\mu_{j}-\gamma r_{j}\right]}{z}\right)^{\alpha} I_{\mu_{j}-\gamma r_{j} \leq z}=\sum_{j=1}^{N} \frac{k}{k N}\left(\frac{z-\left[\mu_{j}-\gamma r_{j}\right]}{z}\right)^{\alpha} I_{\mu_{j}-\gamma r_{j} \leq z}, \alpha \geqslant 0
$$

\footnotetext{
${ }^{3}$ Para detalles sobre la relevancia que ha tenido esta clase de índices en la literatura de pobreza, véase: Foster et al.(2010).
} 
expresión que continua siendo igual a (2.4).

4. Sea $h_{j}=\mu_{j}-\gamma r_{j}, \forall j$. Sea h el vector de valores de todos los $h_{j}$ en la población, es decir: $\mathrm{h}=\left(h_{1}, h_{2}, . ., h_{N}\right)$. Sabemos que $\mu_{j}$ y $r_{j}$ son números reales, para todo $j$, ya que son parámetros de una variable aleatoria. Sabemos también que $\gamma$ es un número real en el intervalo $(0,1]$, entonces $h_{j}$ es también un número real para todo $j$. Pero ya Foster et al.(1984: 763) demostraron, que para valores reales de $h_{j}$, el siguiente índice cumple el axioma 6 para $\alpha>0$, el axioma 7 para $\alpha>1$ y el axioma 8 para $\alpha>2$ :

$$
V_{\alpha}(\mathrm{h}, z)=\sum_{j=1}^{N} \frac{1}{N}\left(\frac{z-h_{j}}{z}\right)^{\alpha} I_{h_{j} \leq z}, \alpha \geqslant 0
$$

Entonces, la medida de vulnerabilidad $V_{\alpha}$ definida en (2.4), cumple exactamente los mismos axiomas para los valores indicados de $\alpha$, ya que la expresión (2.6) es igual a la expresión (2.4).

5. Sabemos que $h_{j}=\mu_{j}-\gamma r_{j} \geq 0$, ya que $y_{j} \geq 0$. Entonces, el mínimo valor que puede tomar $h_{j}$ es cero. Luego, por la condición de foco, la medida $V_{\alpha}$ contiene solamente valores de $h_{j}$ tales que: $h_{j} \leq z$. Por lo tanto, el máximo valor que puede tomar $h_{j}$ es $z$. Entonces, $\frac{z-h_{j}}{z} \in[0,1], \forall j$. Luego, $\left(\frac{z-h_{j}}{z}\right)^{\alpha}, \alpha \geq 0$, también pertenece al intervalo [0, 1], y entonces $V_{\alpha} \in[0,1]$, al ser una suma ponderada de valores contenidos en el intervalo $[0,1]$. 6. Para todo número real $\eta \in[0, z]$ y para todo $\alpha>0$, se cumple que $\lim _{h_{j} \rightarrow \eta}\left(\frac{z-h_{j}}{z}\right)^{\alpha}=$ $\left(\frac{z-\eta}{z}\right)^{\alpha}$. De modo que la función: $f\left(h_{j}\right)=\left(\frac{z-h_{j}}{z}\right)^{\alpha}$ es continua para $\alpha>0$. Mientras tanto, para $\alpha=0$ la función $f\left(h_{j}\right)=\left(\frac{z-h_{j}}{z}\right)^{\alpha}$ es constante e igual a uno, por lo que también es continua, excepto en el punto $z$, donde esta función no está definida, ya que $0^{0}$ es indeterminado. Luego, al ser $V_{\alpha}$ una suma ponderada de funciones continuas, también es continua, excepto en la línea de pobreza para $\alpha=0$.

q.e.d. 
Adicionalmente $V_{\alpha}(\mathrm{h}, z)$ permite la siguiente útil descomposición para propósitos de políticas públicas: ${ }^{4}$

$$
\begin{gathered}
V_{\alpha}=V_{\alpha}^{S}+V_{\alpha}^{M} \\
V_{\alpha}^{S}=\sum_{j=1}^{N} \frac{1}{N}\left(\frac{z-h_{j}}{z}\right)^{\alpha} I_{\mu_{j} \leq z}, \alpha \geqslant 0 \\
V_{\alpha}^{M}=\sum_{j=1}^{N} \frac{1}{N}\left(\frac{z-h_{j}}{z}\right)^{\alpha} I_{\mu_{j}>z \wedge h_{j} \leq z}, \alpha \geqslant 0
\end{gathered}
$$

Donde $V_{\alpha}^{S}$ es la vulnerabilidad severa y $V_{\alpha}^{M}$ es la vulnerabilidad moderada. Mientras que $I_{\mu_{j} \leq z}$ y $I_{\mu_{j}>z \wedge h_{j} \leq z}$ son las funciones indicadoras que satisfacen las correspondientes condiciones de foco para ambos tipos de vulnerabilidad.

\subsection{Una ilustración empírica para el Perú}

\subsubsection{Motivación del ejemplo y datos utilizados}

Perú es un país que se presenta particularmente atractivo para realizar estudios de pobreza y vulnerabilidad, debido a que ha experimentado cambios macroeconómicos importantes, ha estado expuesto a gran variedad de shocks y recientemente ha mostrado logros sustantivos en materia de superación de la pobreza. En la literatura especializada ya se acumula además cierta tradición de realizar estudios sobre vulnerabilidad usando datos de panel de este país (Glewwe y Hall, 1998 y Calvo, 2008). Motivados por estas razones, hemos decidido ilustrar el ejemplo del cálculo del índice de vulnerabilidad a la pobreza propuesto, con datos del Perú.

Los datos utilizados corresponden al panel 2007-2010, de la Encuesta Nacional de Hogares sobre Condiciones de Vida y Pobreza (ENAHO), la cual es implementada por el Instituto Nacional de Estadistica e Informática (INEI). La Encuesta ENAHO ha contado con la asistencia técnica de instituciones internacionales que han puesto su empeño en garantizar la calidad de los datos obtenidos.

El período de observación escogido se presenta particularmente interesante para realizar el ejercicio empírico, ya que en los años 2007-2010 Perú experimentó un fuerte crecimiento económico, con incrementos anuales del PIB real en torno al 7\%, en promedio.

\footnotetext{
${ }^{4}$ De aquí en adelante utilizaremos la expresión del índice definida en (2.6), en lugar de la definida en $(2.4)$.
} 
Pero a la vez estuvo expuesto a la crisis financiera internacional, que afectó la economía del país en el año 2009. ${ }^{5}$ El crecimiento económico repercutió en una importante disminución de la pobreza observada. Según cifras oficiales del INEI (2012), la tasa de incidencia de la pobreza disminuyó 11.6 puntos porcentuales durante el período, pasando de $42.4 \%$ en el año 2007 a $30.8 \%$ en el 2010.

El panel de la Encuesta ENAHO 2007-2010 contiene 2,561 hogares, entrevistados en cuatro períodos anuales consecutivos. Se trata de un panel balanceado que contiene los datos necesarios para el ejercicio, incluyendo las líneas de pobreza oficiales ajustadas a precios regionales y los factores de expansión a la población. Los datos del panel se encuentran libremente disponibles al público en el sitio Web del INEI, por lo cual el cálculo que presentaremos a continuación puede ser reproducido por cualquiera.

La variable focal utilizada en el cálculo es el gasto de consumo per cápita del hogar en cada período: $y_{j t}$. A pesar de que usamos las líneas oficiales de pobreza y los factores de expansión de la encuesta, los resultados del cálculo de pobreza no coincidirán con las cifras oficiales de pobreza del INEI, ya que tales cálculos se realizan con los datos de la Encuesta ENAHO de corte transversal, que por supuesto posee una muestra mayor. A pesar de ello los resultados obtenidos con los datos del panel resultan ilustrativos respecto de la evolución reciente de la vulnerabilidad a la pobreza en ese país.

\subsubsection{Estrategia de estimación empírica}

En la literatura sobre vulnerabilidad a la pobreza destacan dos tipos de estrategias para la estimación del valor esperado de la variable focal de bienestar. La primera de ellas consiste en condicionar la estimación a un set de variables explicativas (usualmente aquellas que se consideran determinantes de la pobreza, más otras variables demográficas de control). Tal estrategia tiene la ventaja de que puede implementarse con datos de corte transversal (Chaudhuri et al., 2002; Suryahadi y Sumarto, 2003; Imai et al., 2011, entre otros) los cuales se encuentran disponibles para la mayoría de países en desarrollo. Sin embargo, tal método de estimación tiene también algunas desventajas. La principal de ellas es que tal enfoque no permite explotar la dimensión temporal para estimar la varianza de la variable focal. Otras dos desventajas de esta estrategia, es que no permite

\footnotetext{
${ }^{5}$ En el año 2009 el crecimiento del PIB real en el Perú fue inferior al 1\%, como resultado del impacto de la crisis financiera internacional. Sin embargo la economía se recuperó rápidamente en el 2010, con un crecimiento real de la actividad que alcanzó el $8.8 \%$ anual.
} 
controlar por los efectos individuales no observables y que en la estimación condicional queda abierta siempre a cierta arbitrariedad en la elección de las variables explicativas.

Una segunda estrategia ha sido estimar la media no condicional para cada observación, explotando la dimensión temporal con datos de panel. Tal ha sido el camino seguido por Ligon y Schechter (2003) y luego por Calvo (2008). ${ }^{6}$ La principal desventaja de este segundo enfoque es la frecuente falta de disponibilidad de tales datos para los países en desarrollo. Además, cuando tales datos están disponibles, lo están usualmente en muestras pequeñas en comparación con los tamaños muestrales de corte transversal. Este es un problema no menor, ya que dificulta el uso amplio de esta técnica para los países en desarrollo.

Sin embargo, la segunda estrategia de estimación se presenta más adecuada para estimar el índice de vulnerabilidad que aquí se propone, ya que permite efectuar las estimaciones controlando por las características no observables que determinan la media individual. El problema que surgiría si estimásemos la media condicional a un set de variables determinantes de la pobreza, sin controlar por las características no observables, es que las diferencias entre la media condicional y la media individual llevarían a un sesgo en las estimaciones de la semi-desviación típica a la baja. Para aclararlo mejor proponemos el siguiente ejemplo. Supongamos un hogar cuya media específica de la variable focal es $\mu_{j}=0,2 z$, donde $z$ es como siempre la línea de pobreza. Si este hogar tiene un riesgo individual de caer bajo la media igual a $r_{j}=0,1 z$, entonces, para $\gamma=1$, la variable $h_{j}=\mu_{j}-\gamma r_{j}$ de este hogar toma el valor $0,1 z$. Pero supongamos que los demás hogares de la muestra que comparten sus atributos observables tienen una media mayor, de manera que hemos estimado una media condicional a un vector de variables $\mathrm{x}_{\mathrm{j}}$, igual a $\widehat{\mu}_{j} \mid \mathrm{x}_{\mathrm{j}}=0,5 \mathrm{z}$. Entonces, el estimador de $r_{j} \mid \mathrm{x}_{\mathrm{j}}$ para este hogar estaría sobrestimado. Es fácil también pensar en un ejemplo en que el estimador de $r_{j}$ se encuentre subestimado, si la media individual fuese grande en comparación con la condicional.

Por otra parte, la estrategia empírica de estimación con datos de panel permite incorporar en la estimación una tendencia individual. Esto último es sumamente relevante, teniendo en cuenta que la vulnerabilidad es un concepto que se enfoca en los resultados esperados ex ante.

En nuestra aplicación seguimos el diseño econométrico propuesto por Calvo (2008)

\footnotetext{
${ }^{6}$ Como veremos a continuación, Calvo (2008) condiciona la estimación solamente en una tendencia temporal específica para cada hogar y en un shock temporal común.
} 
para datos de panel, pero introduciendo una modificación. La especificación de Calvo (2008: 1017) es la siguiente:

$$
y_{j t}=u_{j}+\beta_{j} t+\eta_{t}+\varepsilon_{j t}
$$

donde $u_{i}$ es el efecto individual específico de cada hogar; $\beta_{i}$ es un parámetro que captura la influencia de la tendencia específica de cada hogar sobre la media; $\eta_{t}$, es un efecto temporal específico y $\varepsilon_{j t}$ es el componente aleatorio de error. La incorporación de una tendencia temporal específica para cada hogar, se justifica en que durante el período de observación los hogares pueden estar inmersos en procesos dinámicos de crecimiento o disminución en sus niveles de bienestar de largo plazo. Tales procesos dinámicos pueden estar determinados ya sea por la fase que enfrentan los perceptores del hogar en el ciclo de vida, por procesos de acumulación o desacumulación de capital físico o humano, o por otras razones.

La modificación que introducimos a la regresión especificada en (2.8) es excluir los shocks temporales comunes, al considerar que los cambios en el estado general de la economía forman parte del riesgo que los hogares enfrentan y no de la media. Desde nuestra perspectiva el efecto temporal sobre la media ya es capturado por el componente de tendencia individual. De manera que en nuestra aplicación empírica estimamos simplemente la media no condicional alrededor de una tendencia específica para cada hogar, del siguiente modo:

$$
y_{j t}=u_{j}+\beta_{j} t+\varepsilon_{j t}
$$

Donde la estimación se realiza conforme una versión del modelo de coeficientes aleatorios desarrollado inicialmente por Swamy (1970). ${ }^{7}$ Para controlar por las diferencias temporales y regionales en los precios y en las líneas de pobreza nominales, la estimación de (2.9) la realizamos con los valores normalizados por las líneas de pobreza a los precios temporales y regionales correspondientes.

Los estimadores muestrales de $\mu_{j t} \mathrm{y} \sigma_{j}^{-}$obtenidos para el cálculo del índice de vulnerabilidad de la población son respectivamente los siguientes:

\footnotetext{
${ }^{7}$ La estimación se realiza en Stata implementando un modelo jerárquico sin componentes fijos Es decir, solo con la parte aleatoria. La matriz varianza-covarianza es determinada por los mismos datos. Es decir, se codifica la versión "no estructurada".
} 


$$
\begin{gathered}
\widehat{\mu}_{j t}=\widehat{u}_{i}+\widehat{\beta}_{i} t \\
\widehat{\sigma}_{j}^{-}=\sqrt{\frac{\sum_{t=1}^{T} \operatorname{Min}\left[\left(y_{j t}-\widehat{\mu}_{j t}\right), 0\right]^{2}}{T}}
\end{gathered}
$$

Donde $T$ es el número de observaciones temporales del panel.

\subsubsection{Principales Resultados}

En la Tabla 1 se presentan las medidas FGT de pobreza y de vulnerabilidad a la pobreza, obtenidas a partir del procedimiento descrito anteriormente, considerando un coeficiente de trade-off: $\gamma=1$.

Los cálculos muestrales fueron expandidos a la población usando los factores de expansión presentes en la base de datos del panel ENAHO 2007-2010.

Nótese que los índices FGT de la vulnerabilidad a la pobreza pueden ser interpretados, según el valor de $\alpha$, de manera similar a los índices FGT de pobreza. Es decir, el indicador de vulnerabilidad para $\alpha=0$ corresponde a la tasa de incidencia de la vulnerabilidad a la pobreza; para $\alpha=1$ corresponde a la brecha promedio de vulnerabilidad a la pobreza; mientras que para $\alpha=2$ corresponde a la medida de profundidad de la vulnerabilidad a la pobreza. La facilidad de interpretación del indicador propuesto en (2.4) le brinda una ventaja muy importante en comparación con los indicadores de Ligon y Schechter (2003) y Calvo y Dercon $(2005,2007)$, los cuales son más difíciles de interpretar. ${ }^{8}$

Observamos que tanto la pobreza, como la vulnerabilidad a la pobreza experimentaron reducciones importantes y estadísticamente significativas durante el período de observación. La tasa de incidencia de la vulnerabilidad severa disminuyó en 0.06, en forma muy similar a la reducción experimentada en la tasa de incidencia de la pobreza.

De acuerdo con los resultados, un logro social importante del período, fue la reducción de la brecha y de la profundidad de la vulnerabilidad severa. La primera medida se redujo de 0.123 a 0.074 durante el período, mientras que la segunda medida se redujo un poco más de la mitad. Al juzgar por los errores estándares obtenidos por remuestreo, vemos que tales cambios fueron estadísticamente significativos.

\footnotetext{
${ }^{8} \mathrm{El}$ primero de ellos porque es un indicador medido en útiles; el segundo, porque es un indicador cuya magnitud depende del parámetro de concavidad del índice de Chakravarty (1983).
} 
Por su parte, la vulnerabilidad moderada se mantuvo estable y a niveles bajos, tanto en incidencia, como en brecha y en profundidad. Una consecuencia importante que deriva de este último resultado es que el costo per cápita de asegurar a los hogares de bajo consumo para evitar que caigan bajo sus niveles de bienestar esperado es relativamente bajo, puesto que la brecha de la vulnerabilidad moderada (0.011) es aproximadamente un $1 \%$ de la canasta definitoria de la línea de pobreza.

Tabla 2.1: Medidas FGT de Pobreza y Vulnerabilidad, Perú (2007-2010).

\begin{tabular}{|c|c|c|c|c|}
\hline & 2007 & 2008 & 2009 & 2010 \\
\hline \multicolumn{5}{|c|}{ Pobreza } \\
\hline \multirow[t]{2}{*}{$P_{0}$} & 0.337 & 0.319 & 0.308 & 0.280 \\
\hline & $(0.0108)$ & $(0.0109)$ & $(0.0104)$ & $(0.0105)$ \\
\hline \multirow[t]{2}{*}{$P_{1}$} & 0.111 & 0.101 & 0.089 & 0.076 \\
\hline & $(0.0040)$ & $(0.0039)$ & $(0.0039)$ & $(0.0035)$ \\
\hline \multirow[t]{2}{*}{$P_{2}$} & 0.051 & 0.044 & 0.038 & 0.032 \\
\hline & $(0.0023)$ & $(0.0021)$ & $(0.0021)$ & $(0.0019)$ \\
\hline \multicolumn{5}{|c|}{ Vulnerabilidad } \\
\hline \multirow[t]{2}{*}{$V_{0}$} & 0.404 & 0.373 & 0.351 & 0.328 \\
\hline & $(0.0120)$ & $(0.0121)$ & $(0.0117)$ & $(0.0121)$ \\
\hline \multirow[t]{2}{*}{$V_{1}$} & 0.137 & 0.114 & 0.098 & 0.085 \\
\hline & $(0.0056)$ & $(0.0042)$ & $(0.0041)$ & $(0.0047)$ \\
\hline \multirow[t]{2}{*}{$V_{2}$} & 0.064 & 0.048 & 0.038 & 0.031 \\
\hline & $(0.0041)$ & $(0.0024)$ & $(0.0023)$ & $(0.0030)$ \\
\hline \multicolumn{5}{|c|}{ Vulnerabilidad Severa } \\
\hline \multirow[t]{2}{*}{$V_{0}^{S}$} & 0.278 & 0.258 & 0.239 & 0.219 \\
\hline & $(0.0121)$ & $(0.0117)$ & $(0.0114)$ & $(0.0114)$ \\
\hline \multirow[t]{2}{*}{$V_{1}^{S}$} & 0.123 & 0.103 & 0.087 & 0.074 \\
\hline & $(0.0057)$ & $(0.0042)$ & $(0.0042)$ & $(0.0047)$ \\
\hline \multirow[t]{2}{*}{$V_{2}^{S}$} & 0.062 & 0.047 & 0.037 & 0.029 \\
\hline & $(0.0041)$ & $(0.0024)$ & $(0.0023)$ & $(0.0029)$ \\
\hline \multicolumn{5}{|c|}{ Vulnerabilidad Moderada } \\
\hline \multirow[t]{2}{*}{$V_{0}^{M}$} & 0.126 & 0.115 & 0.112 & 0.110 \\
\hline & $(0.0083)$ & $(0.0090)$ & $(0.0078)$ & $(0.0079)$ \\
\hline \multirow[t]{2}{*}{$V_{1}^{M}$} & 0.014 & 0.012 & 0.011 & 0.011 \\
\hline & $(0.0011)$ & $(0.0009)$ & $(0.0007)$ & $(0.0008)$ \\
\hline \multirow[t]{2}{*}{$V_{2}^{M}$} & 0.002 & 0.002 & 0.002 & 0.002 \\
\hline & $(0.0009)$ & $(0.0002)$ & $(0.0002)$ & $(0.0007)$ \\
\hline
\end{tabular}

Nota: Los subíndices corresponden a los valores de $\alpha$ para cada medida FGT. Los errores estándares en paréntesis fueron obtenidos por remuestreo.

Fuente: Cálculos propios con datos del panel de la encuesta de hogares ENAHO, 2007-2010. 


\subsubsection{Ejercicios de robustez: comparación con los resultados de otros indicadores}

En la tabla 2.2, a continuación se ofrece una comparación de resultados de estimaciones de la vulnerabilidad a la pobreza con indicadores alternativos y para distintos parámetros de aversión al riesgo.

Por una parte se presenta la incidencia de la vulnerabilidad a la pobreza según el índice de semi-desviación típica desarrollado en este capítulo, calculado para otros parámetros de trade-off entre media y riesgo. Conforme los resultados que arroja este indicador vemos que la vulnerabilidad a la pobreza en el Perú ha disminuido para el período considerado, independientemente de cual sea el coeficiente de trade-off seleccionado. Por supuesto, la incidencia de la vulnerabilidad es menor entre menor sea la aversión al riesgo.

En la misma tabla se presentan los resultados de la media de vulnerabilidad para cada año, según el indicador propuesto por Calvo y Dercon (2007) que ya fue descrito en el capítulo anterior. Este indicador también da cuenta de una reducción de la vulnerabilidad a la pobreza en el Perú durante el período de observación, independiente de la magnitud del parámetro de aversión al riesgo, que en este caso corresponde al coeficiente $\rho$ del índice de pobreza de Chakravarty (1983). ${ }^{9}$

Finalmente se ofrecen los cálculos de incidencia de la vulnerabilidad a la pobreza conforme el indicador de Ligon y Schechter (2003), ${ }^{10}$ considerando tres parámetros de aversión al riesgo para la siguiente función de utilidad CRRA (Aversión Relativa al Riesgo Constante) $)^{11}$ :

$$
\left\{\begin{array}{c}
U\left(y_{j}\right)=\frac{y_{j}^{1-\tau}}{1-\tau}, \tau \neq 1 \wedge \tau>0 \\
U\left(y_{j}\right)=\log \left(y_{j}\right), \tau=1
\end{array}\right.
$$

Un inconveniente de este indicador es que no permite capturar la dinámica de la vulnerabilidad año a año, sino que solamente evalúa la vulnerabilidad del período.

La comparación de magnitudes de la vulnerabilidad a la pobreza entre estos indicadores carece de sentido, ya que por un lado están enfocados en aspectos diferentes y tienen distintas formas funcionales. Pero además porque estas medidas varían en dependencia

\footnotetext{
${ }^{9}$ Como ya se indicó en el capítulo anterior, el indicador de Calvo y Dercon (2007) tiene la siguiente especificación individual: $V_{(\rho)}=1-E\left[\frac{\operatorname{Min}\left\{y_{j}, z\right\}}{z}\right]^{\rho}, \rho \in(0,1)$, donde $\rho$ es el parámetro de riesgo.

${ }^{10} \mathrm{El}$ indicador de Ligon y Schechter (2003) para cada hogar, tiene la forma: $V U E_{j}=U_{j}(z)-E\left[U_{j}\left(c_{j}\right)\right]$.

${ }^{11}$ La denominación CRRA corresponde a las siglas en inglés de Constant Relative Risk Aversion
} 
de los parámetros de riesgo: $\gamma, \rho \mathrm{y} \tau$.

Tabla 2.2: Medidas alternativas de Vulnerabilidad para diferentes parámetros de riesgo, Perú (2007-2010).

\begin{tabular}{lcccc}
\hline & $\mathbf{2 0 0 7}$ & $\mathbf{2 0 0 8}$ & $\mathbf{2 0 0 9}$ & $\mathbf{2 0 1 0}$ \\
\hline \hline Índice de semidesviación típica & & & & \\
$\gamma=0,5$ & 0.342 & 0.310 & 0.289 & 0.270 \\
$\gamma=0,75$ & 0.365 & 0.341 & 0.315 & 0.302 \\
$\gamma=1$ & 0.404 & 0.373 & 0.351 & 0.328 \\
Índice de Calvo y Dercon & & & & \\
$\rho=0,25$ & 0.022 & 0.018 & 0.015 & 0.012 \\
$\rho=0,5$ & 0.042 & 0.035 & 0.028 & 0.023 \\
$\rho=0,75$ & 0.059 & 0.049 & 0.041 & 0.033 \\
Índice de Ligon y Shechter & & & & \\
$\tau=1$ & 0.108 & 0.108 & 0.108 & 0.108 \\
$\tau=2$ & 0.172 & 0.172 & 0.172 & 0.172 \\
$\tau=3$ & 0.315 & 0.315 & 0.315 & 0.315 \\
\hline
\end{tabular}

Fuente: Cálculos propios con datos del panel de la encuesta de hogares ENAHO, 2007-2010.

Nota: para el estimador índice de Ligon y Schechter, la estimación es la misma durante todo el período, debido al diseño de este indicador.

\subsection{Conclusiones}

En este capítulo se ha introducido la aplicación de un enfoque asimétrico de mediariesgo (tomado de la literatura de finanzas), al análisis de la pobreza bajo incertidumbre.

Los resultados aleatorios del bienestar de los hogares se han ordenado mediante un criterio de dominancia en media-riesgo, en forma similar a como se ordenan los retornos inciertos de los activos financieros. Tal ordenación ha permitido identificar a los hogares vulnerables a la pobreza, al comparar los resultados aleatorios de su bienestar con la línea de pobreza bajo certidumbre.

Mediante el mismo enfoque de media-riesgo se han distinguido dos tipos de vulnerabilidad a la pobreza: la vulnerabilidad severa o de primer momento vinculada a una situación de pobreza esperada y la vulnerabilidad moderada o de segundo momento, vinculada al riesgo de caer en pobreza debido a desviaciones del bienestar bajo su valor esperado.

Para medir la vulnerabilidad a la pobreza de la población total se ha propuesto un índice tipo FGT que utiliza la semi-desviación típica a la baja como parámetro de riesgo. El cálculo del índice no requiere supuestos sobre las distribuciones de probabilidad de 
la variable focal, sino solamente estimaciones de las medias muestrales y de las semidesviaciones típicas a la baja.

El diseño FGT del índice propuesto garantiza el cumplimiento de la base axiomática deseada, común a los indicadores de pobreza de esta clase.

El cálculo del índice se ha ilustrado en un ejemplo empírico con datos de panel para el Perú. En tal ejemplo se ha mostrado que el cálculo del índice propuesto es simple y que este presenta un potencial muy prometedor para ser utilizado con propósitos de políticas públicas.

\section{Referencias}

Amin, S., Rai A., Topa, G. (2003). "Does microcredit reach the poor and vulnerable? Evidence from Northern Bangladesh". Journal of Development Economics, 70(1): 59-82.

Bawa, V., Lindenberg, E. (1977). "Capital market equilibrium in a mean-lower partial moment framework". Journal of Financial Economics, 5: 189-200.

Calvo, C., Dercon, S. (2005). "Measuring Individual Vulnerability". University of Oxford, Department of Economics Discussion Paper Series, 229.

Calvo, C., Dercon, S. (2007). "Vulnerability to poverty". CSAE Working Paper, 200703.

Calvo, C. (2008). "Vulnerability to Multidimensional Poverty: Peru, 1998-2002". World Development, 36(6): 1011-1020.

Chakravarty, S. (1983). "A new index of poverty". Mathematical Social Sciences, 6(3): 307-313.

Chaudhuri, S., Jalan, J., Suryahadi, A. (2002). "Assessing household vulnerability to poverty from cross-sectional data: a methodology and estimates from Indonesia". Department of Economics Discussion Paper Series, 0102-52, Columbia University.

Christiansen, L. y Subbarao, K. (2005). "Toward an understanding of vulnerability in rural Kenya". Journal of African Economies, 14(4): 540-558.

Dercon, S., Krishnan, P. (2000). "Vulnerability, seasonality and poverty in Ethiopia". Journal of Development Studies, 36(6), 25-53.

Estrada, J. (2002). "Systematic risk in emerging markets: the D-CAPM". Emerging Markets Review, 3, 365-379. 
Foster, J., Greer, J., Thorbecke, E. (1984). "A class of decomposable poverty measures". Econometrica, 52, 761-766.

Foster, J., Greer, J., Thorbecke, E. (2010). "The Foster-Greer-Thorbecke (FGT) poverty measures: 25 years later". Journal of Economic Inequality. 8(4): 491-524.

Gallardo, M. (2013). "Using the downside mean-semideviation for measuring vulnerability to poverty". Economics Letters, 120(3): 416-418.

Glewwe, P., Hall G. (1998). "Are some groups vulnerable to macroeconomic shocks than others? Hypothesis tests based on panel data from Peru", Journal of Development Economics, 56(1): 181-206.

Harlow, V., Rao, R. (1989). "Asset pricing in a generalized mean-lower partial moment framework: theory and evidence". Journal of Financial and Quantitative Analysis, 24: 285-311.

Hoddinott, J., Quisumbing, A. (2003). "Methods of microeconometric risk and vulnerability assessments". SPDP Series 0324, World Bank.

Hogan, W., Warren, J. (1974). "Toward the development of an equilibrium capitalmarket model based on semivariance". Journal of Financial and Quantitative Analysis, 9: $1-11$.

INEI (2012). "Informe técnico: evolución de la pobreza 2007-2011". http://www.inei.gob.pe /DocumentosPublicos/Pobreza_InformeTecnico.pdf.

Imai, K., Gaiha, R., Kang, W. (2011). "Vulnerability and poverty dynamics in Vietnam". Applied Economics, 43(25): 3603-3618.

Kakwani, N. (1980). "On a class of poverty measures". Econometrica, 48(2): 437-446.

Klasen, S., Waibel, H. (2013). "Defining and Measuring Vulnerability: State of the Art and New Proposals". Capítulo 2 del libro: Vulnerability to Poverty: Theory, Measurement and Determinants, with Case Studies from Thailand and Vietnam, Palgrave Macmillan. Editado por: Klasen, S., Waibel, H..

Ligon, E., Schechter, L. (2003). "Measuring vulnerability". Economic Journal, 113(486): C95-C102.

Markowitz, H. (1959). Portfolio Selection. Wiley, New York.

Markowitz, H. (1987). Mean-Variance Analysis in Portfolio Choice and Capital Markets. Blackwell, Oxford.

Morduch, J. (1984). "Poverty and Vulnerability". The American Economic Review, 
84(2): 221-225.

Ogryczak, W., Ruszczynski, A. (1999). "From stochastic dominance to mean-risk models: Semideviations as risk measures". European Journal of Operational Research, 116: 33-50.

Ogryczak, W., Ruszczynski, A. (2001). On consistency of stochastic dominance and mean-semideviations models. Mathematical Programming, 89: 217-232.

Schechter, L. (2003). "Vulnerability as a Measure of Chronic Poverty". Chronic Poverty Research Centre (CPRC), Manchester, UK, 17.

Suryahadi, A., Sumarto, S. (2003). "Poverty and vulnerability in Indonesia before and after the economic crisis". Asian Economic Journal, 17(1), 45-64.

Sen, A. (1976). Poverty: an ordinal approach to measurement. Econometrica, 44(2), 219-231.

Sen, A. (1979). "Issues in the Measurement of Poverty". Scandinavian Journal of Economics, 81: 285-307.

Swamy, P. (1970). "Efficient inference in a random coefficient regression model". Econometrica, 38(2): 311-323. 


\section{Capítulo 3}

\section{Evidencia empírica para América}

\section{Latina}

\section{Resumen}

En este capítulo se realiza un análisis de la evolución reciente de la vulnerabilidad a la pobreza en América Latina, utilizando datos de doce paneles de hogares para cuatro países: Argentina, Perú, Chile y Nicaragua. Se aporta evidencia empírica que valida el uso extensivo del indicador de vulnerabilidad a la pobreza basado en la semi-desviación típica a la baja. De acuerdo con estos resultados, tal indicador permite separar adecuadamente la vulnerabilidad a la pobreza, entre su componente de media y su componente de riesgo. Se encuentra que en general, la reducción de la pobreza en los paneles observados ha ido acompañada de reducciones en la vulnerabilidad, aunque existen períodos en que la pobreza disminuye y la vulnerabilidad permanece estancada al mismo nivel. Una contribución del estudio desarrollado en este capítulo, es mostrar evidencia acerca del siguiente hallazgo empírico: a medida que los países avanzan en la superación de la pobreza, pierde relevancia la vulnerabilidad determinada por la media y cobra mayor relevancia la vulnerabilidad determinada por el riesgo.

\subsection{Introducción}

En la literatura económica se han propuesto una gran diversidad de índices para medir la vulnerabilidad a la pobreza, sin que ninguno de ellos haya conquistado consenso entre 
los que dirigen y ejecutan las políticas públicas. Sin embargo, los enfoques conceptuales sobre este fenómeno han rondado fundamentalmente en torno a dos elementos: (i) el riesgo que enfrentan los hogares de bajos ingresos debido a la aleatoriedad de los resultados del bienestar (Morduch, 1994; Glewwe y Hall, 1998; Dercon y Krishnan, 2000; Amin et al., 2003; Dutta et al., 2011) ó (ii) alguna forma funcional de pobreza esperada (Chaudhuri et al., 2002; Suryahadi y Sumarto, 2003; Christiansen y Subbarao, 2005). Algunos enfoques han intentado también combinar ambos elementos en un sólo indicador (Ligon y Schechter, 2003; Calvo y Dercon 2005, 2007b; Calvo, 2008; Gallardo, 2013).

Entre los enfoques del último tipo, la propuesta de Gallardo (2013) se distingue por el hecho de evaluar el componente de riesgo no a partir de toda la dispersión alrededor del valor esperado del bienestar, sino solamente a partir de la dispersión a la baja, usando para ello la semi-desviación típica como parámetro de riesgo. Bajo tal enfoque, la vulnerabilidad a la pobreza se interpreta como una situación de exposición al riesgo de caer en pobreza. La cual se debe ya sea a que el valor esperado del bienestar del hogar es menor que la línea de pobreza (vulnerabilidad severa), o porque al contabilizar el riesgo de caer bajo el valor esperado del bienestar (a través de la semi-desviación típica a la baja), el hogar se encontraría también eventualmente bajo la línea de pobreza (vulnerabilidad moderada). La propuesta es conceptualmente interesante, pero no posee aún suficiente respaldo empírico, ya que en el trabajo citado, el autor solamente brinda una ilustración del cálculo del nuevo indicador, utilizando un único panel de datos de consumo de hogares del Perú.

Un primer propósito del estudio presentado en este capítulo es someter el indicador de Gallardo (2013) a un examen empírico más riguroso, al aplicar tal enfoque de medición de la vulnerabilidad a la pobreza a una mayor diversidad de datos, en los que se incluyen doce paneles de hogares de cuatro países de América Latina. Con ello se espera obtener evidencia empírica más robusta acerca de las fortalezas y posibles debilidades del nuevo indicador. El estudio además se implementa con paneles de distintas brechas temporales, lo cual permite a su vez contrastar si el indicador resiste la prueba de ser aplicado en tal diversidad de contextos en los set de datos.

Un segundo propósito es contribuir a aportar evidencia empírica sobre la evolución reciente de la vulnerabilidad a la pobreza en América Latina. Los datos de paneles de hogares disponibles en la región son escasos. Mientras que las investigaciones publicadas que han aportado evidencia sobre la vulnerabilidad a la pobreza en el sub continente, 
utilizando este tipo de datos, son más escasas aún. Entre estas últimas apenas se cuenta con los aportes de Glewwe y Hall (1998), Calvo (2008) y Bérgolo et al. (2012). Por ello, los resultados que arroja este trabajo pueden resultar también una contribución empírica interesante para la literatura sobre medición de la vulnerabilidad a la pobreza en América Latina.

El tercer propósito del estudio es contrastar las siguientes hipótesis:

$H_{1}$ : La vulnerabilidad a la pobreza ha disminuido en los paneles de los cuatro países considerados.

$H_{2}$ : A medida que se producen avances de disminución en la tasa de incidencia de la pobreza absoluta, disminuye también la importancia relativa de la tasa de incidencia de la vulnerabilidad severa (la que toma forma de pobreza esperada) y aumenta la importancia relativa de la tasa de incidencia de la vulnerabilidad moderada (la que corresponde al riesgo de caer en pobreza por las desviaciones bajo la media).

La primera hipótesis se sostiene en el supuesto de que si los índices de pobreza han disminuido en el sub continente (CEPAL, 2012), deberíamos esperar que sucediese algo similar con los índices de vulnerabilidad. El contraste de esta hipótesis se realiza construyendo intervalos de confianza para los indicadores de vulnerabilidad, utilizando técnicas de re-muestreo.

Respecto de la segunda hipótesis, ella está relacionada con el hecho estilizado de que el desarrollo económico va acompañado de procesos de incrementos en los ingresos medios de los hogares, lo cual repercute en menores índices de pobreza absoluta (véase por ejemplo: Bourguignon, 2003; Dollar y Kray, 2004). La razón de ello es que ceteris paribus al moverse toda la distribución de ingresos de una población en la dirección al alza, el porcentaje de hogares con ingresos bajo la línea de pobreza absoluta disminuye. Sin embargo, los cambios en el riesgo que enfrentan los hogares para desviarse de su bienestar promedio pueden ser más lentos. En consecuencia, deberíamos esperar que la importancia relativa de la vulnerabilidad severa a la pobreza disminuya más, en comparación con la importancia relativa de la vulnerabilidad moderada.

Si esta segunda hipótesis es válida, observaríamos una mayor importancia relativa de la vulnerabilidad severa a la pobreza en el panel del país más pobre de la muestra (Nicaragua) y una menor importancia relativa de este indicador en el panel del país con menor pobreza (Chile). Con situaciones intermedias evolutivas en los paneles de Perú y 
Argentina. Para contrastar esta segunda hipótesis, además de la evidencia observacional se presentan tres contrastes estadísticos simples: un contraste t-student por regresión lineal semi-logarítmica simple, un ANOVA convencional de efectos fijos y un test no paramétrico: el Kruskal Wallis. En los dos últimos casos los contrastes se implementan agrupando por rangos el factor: tasa de incidencia de la pobreza absoluta.

El trabajo enfrenta también algunas limitaciones. La primera de ellas tiene que ver con que los resultados obtenidos son sólo parcialmente representativos de la población. Algunos de los paneles utilizados tienen problemas de desgaste y en el caso de los 8 paneles de Argentina, dos de ellos tienen muestras más pequeñas. Para los dos paneles del Perú se observa un quiebre en los resultados, lo que hace sospechar de sesgo en el muestreo o sesgo por desgaste de panel. En los casos de Chile y Nicaragua las brechas entre las observaciones temporales de los paneles son grandes (5 y 3-4 años respectivamente) lo cual admite la posibilidad de que los hogares observados hayan sufrido cambios demográficos sustantivos durante el período de observación. Finalmente, las estadísticas de cambios en los precios para algunos países no son fiables, ${ }^{1}$ por lo que se debió recurrir a algunas estimaciones de precios ad hoc para poder estimar adecuadamente una línea de pobreza comparable entre países a paridad de poder de compra.

El capítulo se encuentra estructurado del siguiente modo: en la sección 3.2 se presenta el marco conceptual y la medida de vulnerabilidad utilizada, en la 3.3 se expone la estrategia de estimación empírica, luego en la sección 3.4 se describen los datos utilizados y en la 3.5 se discuten los resultados. Al final se ofrecen las conclusiones correspondientes.

\subsection{Concepto y medida de vulnerabilidad}

En esta sección se resume el enfoque conceptual y de medición de la la vulnerabilidad a la pobreza propuesto en Gallardo (2013). Denotamos como $y_{j}$ a la variable aleatoria que representa el resultado del bienestar del hogar $j$, tal que $y_{j} \geq 0$. Suponemos un escenario en el que desconocemos la distribución de $y_{j}$ para cada hogar. Sin embargo, consideramos que podemos estimar razonablemente su valor esperado $\mu_{j}$ y una medida $r_{j}$, que representa el riesgo de que tal variable focal de bienestar se desvíe por debajo de

\footnotetext{
${ }^{1}$ En el caso de Argentina, el Instituto Nacional de Estadísticas y Censos (INDEC) ha sido duramente criticado tanto en el ámbito académico, periodístico e incluso por organismos internacionales porque sus estadísticas de precios no son creíbles. Véase por ejemplo el informe académico UBA (2010).
} 
$\mu_{j}$. Como medida de riesgo $r_{j}$ adoptamos la semi-desviación típica a la baja, la cual se define del siguiente modo:

$$
\sigma_{j}^{-}=\sqrt{E\left\{\operatorname{Min}\left[\left(y_{j}-\mu_{j}\right), 0\right]^{2}\right\}}
$$

Asumimos que los hogares toman decisiones racionales y que teniendo en cuenta la incertidumbre de los resultados de su bienestar, se comportan con preferencias estrictamente crecientes en $\mu_{j}$ (no saciedad local) y estrictamente decrecientes en $\sigma_{j}^{-}$(aversión estricta al riesgo). De manera que tales preferencias pueden ser representadas razonablemente por una función de utilidad del tipo: $U\left(\mu_{j}, r_{j}\right)$, con derivadas parciales: $\frac{\partial U_{j}}{\partial \mu_{j}}>0, \frac{\partial U_{j}}{\partial r_{j}}<0, \forall j$.

Dadas tales preferencias, para cualquier par de variables aleatorias: $y_{j}, y_{k}$, se cumple la siguiente relación de dominancia estricta en media-riesgo (donde $\succ$ denota dicha relación de comparación racional):

$$
y_{j} \succ y_{k} \Leftrightarrow \mu_{j} \geqslant \mu_{k} \wedge \sigma_{j}^{-} \leqslant \sigma_{k}^{-}
$$

con al menos una de las desigualdades estricta.

Para poder comparar resultados aleatorios del bienestar con distinta media y distinto riesgo, supondremos un coeficiente de trade-off entre media y riesgo: $\gamma>0$. De manera que para cualquier par de variables aleatorias: $y_{j}, y_{k}$, la relación de dominancia en media riesgo se podrá expresar del siguiente modo:

$$
y_{j} \succ y_{k} \Leftrightarrow \mu_{j}-\gamma \sigma_{j}^{-}>\mu_{k}-\gamma \sigma_{k}^{-}
$$

En adelante supondremos que las ganancias en los resultados esperados del bienestar son valoradas con igual ponderación, que el evitar las pérdidas por riesgo. Es decir, asumimos que $\gamma=1$. Ello con el propósito práctico adicional de facilitar la comparación de los resultados entre la vulnerabilidad por pobreza esperada y la vulnerabilidad por riesgo, ya que así ambos tipos de vulnerabilidad estarán siendo ponderados por igual. No obstante, tal coeficiente de trade-off entre media y riesgo no tiene porque necesariamente ser igual a uno. Como se explicó en Gallardo (2013), el coeficiente $\gamma$ debería estar acotado en el intervalo $(0,1]$, pero su elección dependerá del criterio de la autoridad política. O como en nuestro caso: de la decisión del investigador.

De acuerdo con Ogryczak y Ruszczynski (1999, 2001), sabemos además, que la relación 
de dominancia en media-riesgo definida (3.3) es consistente con la dominancia estocástica de segundo orden, para valores acotados de $\gamma$ en el intervalo $[0,1]$.

Bajo tal ordenación de resultados del bienestar de los hogares en incertidumbre, como se define en Gallardo (2013: 3): diremos que el hogar $j$ es vulnerable a la pobreza, si y sólo si: $\mu_{j}-\gamma \sigma_{j}^{-} \leq z$, donde $z$ denota la línea de pobreza bajo certidumbre. Asimismo, diremos que el hogar $j$ sufre vulnerabilidad severa a la pobreza (pobreza esperada o vulnerabilidad de primer momento), sí $\mu_{j} \leq z$; mientras que si $\mu_{j}>z$, pero $\mu_{j}-\gamma \sigma_{j}^{-} \leq z$, diremos que el hogar se encuentra en situación de vulnerabilidad moderada a la pobreza (vulnerabilidad por riesgo, o de segundo momento).

Para medir la vulnerabilidad de la población, utilizaremos el siguiente índice de agregación, que sigue el tradicional diseño FGT (Foster-Greer-Thorbecke, 1984):

$$
V_{\alpha}=\sum_{j=1}^{N} \frac{1}{N}\left(\frac{z-\left[\mu_{j}-\gamma \sigma_{j}^{-}\right]}{z}\right)^{\alpha} I_{\mu_{j}-\gamma \sigma_{j}^{-} \leq z}, \alpha \geqslant 0
$$

Donde $I_{\mu_{j}-\gamma \sigma_{j}^{-} \leq z}$ es una función indicadora que vale uno si el hogar $j$ satisface la condición de foco: $\mu_{j}-\gamma \sigma_{j}^{-} \leq z$, y vale cero si no, mientras que $N$ es el tamaño de la población. El diseño FGT en (3.4) garantiza que en dependencia del valor de $\alpha$, tal índice de vulnerabilidad satisfaga la base axiomática característica de esta clase de índices de pobreza. $^{2}$

Como es sabido, la medida anterior se puede descomponer además en los dos indicadores siguientes:

$$
\begin{gathered}
V_{\alpha}=V_{\alpha}^{S}+V_{\alpha}^{M} \\
V_{\alpha}^{S}=\sum_{j=1}^{N} \frac{1}{N}\left(\frac{z-\left[\mu_{j}-\gamma \sigma_{j}^{-}\right]}{z}\right)^{\alpha} I_{\mu_{j} \leq z}, \alpha \geqslant 0 \\
V_{\alpha}^{M}=\sum_{j=1}^{N} \frac{1}{N}\left(\frac{z-\left[\mu_{j}-\gamma \sigma_{j}^{-}\right]}{z}\right)^{\alpha} I_{\mu_{j}>z \wedge \mu_{j}-\gamma \sigma_{j}^{-} \leq z}, \alpha \geqslant 0
\end{gathered}
$$

donde $V_{\alpha}^{S}$ es la vulnerabilidad severa y $V_{\alpha}^{M}$ es la vulnerabilidad moderada. Mientras que $I_{\mu_{j} \leq z}$ y $I_{\mu_{j}>z \wedge h_{j} \leq z}$ son las funciones indicadoras que satisfacen las correspondientes condiciones de foco para ambos tipos de vulnerabilidad.

Al no contar con datos de toda la población, la estimación de (3.4) debe hacerse con

\footnotetext{
${ }^{2}$ Para detalles, véase: Gallardo (2013).
} 
una muestra, ponderando los cálculos por los correspondientes factores de expansión de las unidades muestrales a la población.

\subsection{Estrategia de estimación empírica}

Para poder calcular el índice que se señala en (3.4) se requieren estimaciones muestrales de dos escalares para cada hogar: la media y la semi-desviación típica a la baja. A su vez, el segundo escalar es dependiente de la media, por lo cual, en este enfoque de vulnerabilidad es crucial la estimación del primer parámetro.

Una alternativa simple es calcular el promedio de la variable focal para cada hogar, en la dimensión temporal. Tal es la estrategia seguida por Ligon y Schechter (2003). Sin embargo, ello no tiene en cuenta los cambios en la media a través del tiempo. Crítica que ya fue formulada por Calvo y Dercon (2007a), en el contexto de su análisis sobre la pobreza crónica y la dinámica de la pobreza. ${ }^{3}$

Una consecuencia de ignorar los cambios en la media a través del tiempo, es que una sola observación bajo la línea de pobreza puede inducirnos a considerar que el hogar se encuentra siempre con una media bajo tal umbral. Ello conduciría a la vez a una sobre estimación de la vulnerabilidad severa a la pobreza. Un caso típico sería el de aquellos hogares que tienen una tendencia en ascenso, partiendo de un resultado muy bajo al inicio. En tal caso, la observación del período inicial desplazaría hacia abajo la estimación de la media de los períodos siguientes. De manera que el cálculo de la vulnerabilidad severa quedaría fuertemente influenciado por tal observación. Lo contrario sucedería con hogares con una tendencia en descenso y con un resultado bajo la línea de pobreza en el período final. Para explicarlo mejor proponemos el siguiente ejemplo: supongamos un hogar cuyos ingresos per cápitas normalizados a la línea de pobreza, en un panel de tres observaciones sean: 0.2, 1.1 y 1.4. Dado que el promedio simple de resultados de este hogar es 0.9, menor que la línea de pobreza (que en este caso es uno), entonces, el hogar sería clasificado como vulnerable en el sentido severo durante las tres observaciones. Es decir, el promedio de su vulnerabilidad en los tres períodos nos arrojaría un valor de $100 \%$ de incidencia y además sería igual al promedio de su vulnerabilidad severa en los tres períodos. Sin embargo, este hogar tiene un resultado bajo la línea de pobreza únicamente en la primera observación.

\footnotetext{
${ }^{3}$ Hogares con distintas trayectorias que cruzan la línea de pobreza pueden tener la misma media en las observaciones de un panel.
} 
Si calculamos su media considerando su evolución en el tiempo, por ejemplo a través de una tendencia lineal (el resultado sería la recta: $\widehat{y}=-0,3+0,6 t$ ), obtendremos en tal caso que la incidencia de su vulnerabilidad en los tres períodos es solamente $32 \%$, con una vulnerabilidad severa promedio de $27 \%$ y una incidencia de la vulnerabilidad moderada de $5 \%$.

Otra alternativa de estimación de la media individual, sugerida por Calvo y Dercon (2007b) fue la de usar un modelo econométrico auto regresivo con datos de panel. Sin embargo, tal estrategia fue desechada pronto por el mismo Calvo (2008). La razón de ello es que se pierde al menos una observación, que es sumamente valiosa para estimar el riesgo, ya que normalmente contamos solamente con paneles cortos.

Mejor fue la propuesta que introdujo Calvo (2008), la cual consiste en estimar el siguiente modelo econométrico con datos de panel:

$$
y_{j t}=u_{j}+\beta_{j} t+\eta_{t}+\varepsilon_{j t}
$$

donde $u_{j}$ es el efecto individual específico de cada hogar; $\beta_{j}$ es un parámetro que captura la influencia de la tendencia específica de cada hogar sobre la media; $\eta_{t}$, es un efecto temporal específico y $\varepsilon_{j t}$ es el componente de error aleatorio. La incorporación de la tendencia temporal específica precisamente tiene en cuenta los procesos dinámicos en que pueden estar inmersos los hogares durante el período de observación.

Acá seguimos un diseño similar, introduciendo una modificación al excluir del modelo el efecto temporal específico. La razón para hacerlo se debe a que consideramos que los cambios en el estado general de la economía deben separarse en dos elementos. Uno de ellos es el componente de tendencia que afecta de manera distinta a cada hogar en su media. Este elemento ya es capturado en forma individual por el parámetro $\beta_{j}$. La otra parte son los cambios generales en la economía que actúan como shocks temporales y que también afectan de manera distinta a cada hogar. Este segundo elemento lo consideramos parte del riesgo que enfrentan los hogares y no de su media.

Nuestro modelo queda especificado entonces del siguiente modo:

$$
y_{j t}=u_{j}+\beta_{j} t+\varepsilon_{j t}
$$


Para los propósitos de esta investigación, este modelo simple tiene además la ventaja de facilitar la comparación internacional. Al usar por ejemplo un modelo condicionado en un set de características observables de los hogares, se enfrentaría el problema de que el set de variables explicativas disponibles es distinto en cada encuesta nacional. Además, lo que acá se pretende es solamente estimar la media de la variable focal para cada hogar, con la intención de calcular la vulnerabilidad a la pobreza. No interesa el efecto marginal de determinada variable explicativa sobre la variable focal.

Un modelo con coeficientes específicos como el planteado en (3.7) puede ser estimado considerando coeficientes fijos (Zellner, 1962), o coeficientes aleatorios (Swamy, 1970) para cada unidad de observación. Sin embargo, como en este caso los valores de las variables explicativas son iguales para todas las observaciones, entonces el modelo de coeficientes fijos, aún considerando errores heterocedásticos, sería equivalente al resultado de estimar una regresión individual para cada hogar por Mínimos Cuadrados Ordinarios (Véase: Hsiao, 2003: 144). Tal estimación arrojaría resultados poco fiables con paneles cortos. Por ejemplo, en paneles de tres observaciones temporales para cada hogar, como son los casos de Chile y Nicaragua en el presente estudio, estimaríamos dos parámetros para cada unidad individual con solo tres observaciones, limitando al extremo los grados de libertad. ${ }^{4}$

La alternativa de coeficientes aleatorios es más adecuada en este contexto, ${ }^{5}$ ya que permite estimar los parámetros aprovechando también la información transversal. De acuerdo con tal modelo, los coeficientes en (3.7) se pueden descomponer del siguiente modo: $u_{j}=u_{0}+u_{0 j}$ y $\beta_{j}=\beta_{0}+\beta_{0 j}$, donde $u_{0 j}$ y $\beta_{0 j}$ son los componentes aleatorios de los coeficientes, mientras que $u_{0}$ y $\beta_{0}$ son sus medias poblacionales. De este modo la expresión en (3.6) toma la siguiente forma:

$$
y_{j t}=\left(u_{0}+u_{0 j}\right)+\left(\beta_{0}+\beta_{0 j}\right) t+\varepsilon_{j t}
$$

donde ahora el vector de parámetros: $\underline{\delta}_{j}=\left(u_{0 j}, \beta_{0 j}\right)$, sigue una distribución normal con media cero y matriz de covarianza: $E\left(\underline{\delta}_{j} \underline{\delta}_{j}^{\prime}\right)=\Delta$, para todo $j$. Mientras que el vector de

\footnotetext{
${ }^{4}$ El autor comprobó que las estimaciones de (3.7) por efectos fijos para Chile y Nicaragua (que no se presentan en este trabajo) arrojan resultados no satisfactorios, sobrestimando la vulnerabilidad a la pobreza en Nicaragua y subestimándola en Chile.

${ }^{5}$ Calvo (2008) utiliza también un modelo de coeficientes aleatorios.
} 
errores $\underline{\varepsilon}_{j}$, tiene media cero y matriz de covarianza $E\left(\underline{\varepsilon}_{j} \underline{\varepsilon}_{j}^{\prime}\right)=\Sigma_{j}$, para cada $j$. Se supone además que la covarianza entre los errores de las distintas unidades de observación es cero: $E\left(\underline{\varepsilon}_{j} \varepsilon_{i}^{\prime}\right)=0$, para $j \neq i$; y que los componentes aleatorios de los coeficientes son exógenos: $E\left(\underline{\delta}_{j} \underline{\varepsilon}_{i}^{\prime}\right)=0$, para todo $i, j$.

Bajo estos supuestos el estimador de Mínimos Cuadrados Ordinarios de (3.7) es insesgado y consistente, pero ineficiente. De manera que la estimación de un modelo como este se lleva a cabo usualmente por Mínimos Cuadrados Generalizados. También es posible realizar la estimación por Máxima Verosimilitud adoptando una estructura flexible de la matriz varianza-covarianza poblacional, permitiendo que los datos la definan. Acá seguimos este segundo método de estimación. En la literatura, la estimación del modelo por este método es una variante de "modelo jerárquico" (véase: Gelman y Hill, 2007), ya que posee dos tipos de parámetros: los específicos para cada j y los llamados "hiperparámetros" que son comunes a la población. Nótese que en este modelo, la esperanza estimada de $y_{j t}$ es: $\widehat{y}_{j t}=\widehat{u}_{j}+\widehat{\beta}_{j} t^{6}$

Por otra parte, para aproximar al máximo la distribución poblacional de los datos al diseño gaussiano requerido por el método de Máxima Verosimilitud, en el proceso de estimación de este estudio se transformó la variable dependiente a logaritmos neperianos y luego se utilizó el procedimiento de Duan (1983) para recuperar el estimador de la media en niveles, utilizando el promedio del error intra-grupos. ${ }^{7}$

La semi-desviación típica a la baja se estimó del siguiente modo:

$$
\widehat{\sigma}_{j}^{-}=\sqrt{\frac{\sum_{t=1}^{T} \operatorname{Min}\left[\left(y_{j t}-\widehat{y}_{j t}\right), 0\right]^{2}}{T}}
$$

\footnotetext{
${ }^{6}$ Como lo señalan Arellano y Bover (1990: 9), el uso extendido de los términos "efectos fijos" y "efectos aleatorios" en la literatura de datos de panel es frecuentemente motivo de confusión. Que los coeficientes sean aleatorios no significa en este caso que sean cambiantes dentro de los grupos, ni tampoco que estén asociados al término de error como es lo usual en el modelo tradicional de componentes de error de Balestra y Nerlove (1966), lo que significa simplemente es que los efectos específicos no se consideran correlacionados con el error $\varepsilon_{j t}$.

${ }^{7}$ Cuando se estima un modelo en logaritmos neperianos y lo que se quiere obtener es una estimación de la media en niveles, es un error aplicar directamente la función exponencial para "recuperar" el estimador de la media en niveles. Ello se debe a que: $\exp \left(E\left(\ln \left(y_{j} \mid \mathrm{x}_{\mathrm{j}}\right)\right) \neq E\left(y_{j} \mid \mathrm{x}_{\mathrm{j}}\right)\right.$. Sin embargo, ya que en un modelo especificado en logaritmos neperianos: $\ln y_{j}=\mathrm{x}_{j}^{\prime} \beta+v_{j}$, implica que: $y_{j}=\exp \left(\mathrm{x}_{j}^{\prime} \beta\right) \exp \left(v_{j}\right)$, entonces: $E\left(y_{j} \mid \mathrm{x}_{\mathrm{j}}\right)=\exp \left(E\left(\ln \left(y_{j} \mid \mathrm{x}_{\mathrm{j}}\right)\right) E\left(\exp \left(v_{j} \mid \mathrm{x}_{\mathrm{j}}\right)\right)\right.$. Así, según el procedimiento de Duan (1983) se puede recuperar el estimador de la esperanza de la variable en niveles, multiplicando el exponencial de la predicción en logaritmos $(\exp (\widehat{E}(\ln (y \mid \mathrm{x}))))$ por el promedio muestral del exponencial del error muestral de la regresión logarítmica $\left(\frac{1}{n} \sum_{j=1}^{n} \exp \left(\widehat{v}_{j}\right)\right)$ (véase: Cameron y Trivedi, 2009: 103). En este caso se usaron los promedios de los exponenciales de los residuos muestrales intra grupos.
} 
donde $T$ es el número de observaciones en la dimensión temporal.

Como la estimación de $\sigma_{j}^{-}$se realiza con una media estimada que cambia en el tiempo, es posible encontrar algunos casos (son pocos) en que la estimación: $\widehat{\mu}_{j}-\gamma \widehat{\sigma}_{j}^{-}$sea menor que cero. Esto sucede cuando para algún hogar, la variable focal toma el valor cero o muy cercano a cero en algún momento del tiempo ${ }^{8}$ y tiene desviaciones bajo una media estimada más alta en otro momento del tiempo. Aunque teóricamente, para variables aleatorias con valores positivos la media siempre es mayor que la semi-desviación a la baja, estos casos pueden darse cuando la semi-desvición es una estimación. Ante tal evento se requiere sustituir $\widehat{\mu}_{j}-\gamma \widehat{\sigma}_{j}^{-}$por cero, ya que el mínimo nivel de bienestar que puede tomar la variable focal es cero. Algo similar sucede cuando se estima la varianza por Mínimos Cuadrados en tres etapas conforme el estimador de Chaudhuri et al. (2002). En tal caso, la estimación de dicho parámetro puede arrojar valores negativos y lo que se hace usualmente ante tal evento es perder observaciones (se eliminan las "anomalías"). Acá en cambio, no es necesario eliminar observaciones, basta simplemente con realizar la sustitución indicada, ya que teóricamente no es posible estar peor que con un nivel de bienestar igual a cero.

\subsection{Descripción de los datos utilizados}

Como indicador de bienestar $y_{j}$ se consideró el ingreso per cápita del hogar en cada período del tiempo. ${ }^{9}$ Por argumentos muy conocidos en la literatura (Deaton, 1997) sería preferible utilizar el consumo como medida de bienestar. ${ }^{10}$ Sin embargo, en los datos de panel de Chile y Argentina no se dispone de mediciones para dicha variable. En cambio los datos de ingreso están disponibles para todos los países analizados, permitiendo así la comparabilidad internacional.

En todos los casos analizados se utilizan datos de paneles balanceados, sin considerar posibles sesgos por desgaste de panel. Los datos se normalizan a la línea de pobreza

\footnotetext{
${ }^{8}$ Por ejemplo, si la variable focal es el ingreso, como en este estudio, en las encuestas de hogares encontraremos datos con ingresos cero o muy cercanos a cero. En la aplicación empírica de Gallardo (2013), tales anomalías no se encontraron, ya que la variable focal de tal estudio fue el consumo per cápita del hogar, que siempre toma valores mayores que cero.

${ }^{9}$ Se elige el ingreso per cápita y no el ingreso equivalente, para esquivar las posibles complicaciones que pueden surgir debido a las diferencias en las escalas de equivalencia de los países, ya que no se cuentan con escalas de equivalencia para todos los países analizados.

${ }^{10} \mathrm{El}$ consumo corriente fluctúa menos que el ingreso corriente y se considera una mejor aproximación al ingreso permanente.
} 
utilizada: cuatro dólares diarios de EEEUU a precios del año año 2005, ajustados a paridad de poder de compra.

Para el caso de Argentina se utiliza la Encuesta de Permanente de Hogares(EPH) trimestral, que realiza el Instituto Nacional de Estadísticas y Censos (INDEC). En esta encuesta no se entrevistan a los mismos hogares en cada trimestre. Sin embargo, los mismos hogares son entrevistados en cuatro oportunidades, dos veces en un año y dos veces en el año siguiente, con brechas entre las entrevistas de un año y las del siguiente, ya que el mismo hogar es entrevistado ya sea en los dos primeros trimestres durante dos años consecutivos, o en los trimestres segundo y tercero e cada año, o en los trimestres tercero y cuarto de dos años. La encuesta no obstante permite armar paneles cortos bianuales, con cuatro observaciones trimestrales cada uno. El que las distancias entre trimestres puedan diferir no es un problema mayor para la estimación, pues en el caso de Argentina los resultados de cada panel bianual se promediaron después de hacer los cálculos. Es decir, los índices de vulnerabilidad corresponden a los promedios obtenidos para cada panel bianual. Con ello se consigue hacer comparables los resultados con el resto de países en la periodicidad anual.

Los datos de Argentina tienen la ventaja de que permiten obtener resultados año a año. Sin embargo, también tienen la desventaja de que no brindan cobertura de información sobre la población rural, como en las encuestas de los otros países. Aunque esto no debería representar un problema mayor, teniendo en cuenta que en Argentina el $92 \%$ de la población habita en zonas urbanas.

Entre los paneles de Argentina hay dos de ellos con menores tamaños muestrales. Ello se explica por lo siguiente: para el año 2003 solamente están disponibles los datos de los trimestres, tercero y cuarto, mientras para el año 2007, hacen falta los datos del tercer trimestre, los cuales no se encuentran disponibles en el sitio Web del INDEC. De manera que las olas: 2003-2004, 2006-2007 y 2007-2008, tienen muestras más pequeñas.

Para el caso del Perú se utilizan dos paneles provenientes de la Encuesta Nacional de Hogares, que realiza anualmente el Instituto Nacional de Estadística e Informática (INEI). El primer panel reúne tres observaciones anuales entre los años 2004-2006, mientras que el segundo comprende cuatro datos anuales entre los años 2007-2010. Con ambos paneles se cubren entonces un total de siete observaciones anuales consecutivas. El tamaño de la muestra del primer panel es de 3,398 hogares y la del segundo panel es de 2,538 hogares. 
Siendo mayor el desgaste del segundo panel debido a su mayor duración. Por consiguiente, el total de observaciones para del primer panel es 13,592 y el del segundo panel es 10,152.

El hecho de que los paneles de hogares del Perú ofrezcan datos anuales consecutivos los hace atractivos para el estudio de la vulnerabilidad a la pobreza. ${ }^{11}$ Primero porque al no existir lagunas en la dimensión temporal, el seguimiento de la evolución de la vulnerabilidad se vuelve más metódico. Segundo, porque la probabilidad de que se produzcan cambios abruptos en la estructura demográfica del hogar entre un año y otro es baja, en comparación con lo que sucede por ejemplo en los paneles de Chile y Nicaragua, que tienen brechas de varios años entre las observaciones.

Los datos para Nicaragua se obtuvieron de la Encuesta Nacional de Hogares sobre Medición del Nivel de Vida (ENMV), realizada por el Instituto Nacional de Información de Desarrollo (INIDE). Este panel está compuesto de tres observaciones en la dimensión temporal, separadas por brechas importantes. Dichas observaciones corresponden a los años 1998, 2001 y 2005. Un problema que presenta este panel es que la primera brecha es de tres años y la segunda de cuatro. Para resolver tal diferencia de brechas temporales la variable de tendencia en el caso de Nicaragua tiene una distancia entre observación proporcional a las brechas (toma valores 1, 2 y 3.33). El tamaño de la muestra utilizada de este panel es de 1,921 hogares, que suman un total de 5,763 observaciones.

En el caso de Chile, los datos provienen de la Encuesta Panel CASEN que realiza el Observatorio Social de la Universidad Alberto Hurtado. Se trata de un panel con observaciones temporales para los años 1996, 2001 y 2006. La muestra utilizada está compuesta por 3,662 hogares, sumando un total de 10,986 observaciones. Este panel posee la limitación de tener brechas largas entre cada observación sobre la dimensión temporal. Esto último hace posible la existencia de cambios demográficos importantes al interior de las unidades observadas, lo cual potencialmente es un elemento distorsionador para el análisis.

Las estadísticas descriptivas de los datos utilizados se presentan a continuación en la tabla 3.1.

\footnotetext{
${ }^{11}$ En los destacados artículos sobre vulnerabilidad de Glewwe y Hall (1998) y Calvo (2008), se utilizaron datos de paneles del Perú.
} 
Tabla 3.1: Estadísticas descriptivas del ingreso per cápita de los hogares, normalizado por la línea de pobreza.

\begin{tabular}{lccccccc}
\hline País & Período & Obs. & Media & Desv. típica & Asimetría & Mín. & Máx. \\
\hline \hline Argentina & $2003-2004$ & 2406 & 2.3 & 2.9 & 5.8 & 0.00 & 57.3 \\
Argentina & $2004-2005$ & 7562 & 2.6 & 3.1 & 9.0 & 0.00 & 146.9 \\
Argentina & $2005-2006$ & 7550 & 2.9 & 3.8 & 17.6 & 0.00 & 276.6 \\
Argentina & $2006-2007$ & 2389 & 3.0 & 3.3 & 5.3 & 0.00 & 76.7 \\
Argentina & $2007-2008$ & 3295 & 3.1 & 3.4 & 6.9 & 0.00 & 113.5 \\
Argentina & $2008-2009$ & 9782 & 3.6 & 4.3 & 14.2 & 0.00 & 286.0 \\
Argentina & $2009-2010$ & 9684 & 3.5 & 4.8 & 49.3 & 0.00 & 585.8 \\
Argentina & $2010-2011$ & 9620 & 3.9 & 4.0 & 4.6 & 0.00 & 109.5 \\
Perú & 2004 & 3398 & 1.7 & 2.1 & 9.0 & 0.03 & 54.7 \\
Perú & 2005 & 3398 & 1.9 & 3.6 & 25.3 & 0.04 & 155.7 \\
Perú & 2006 & 3398 & 2.1 & 3.2 & 12.5 & 0.08 & 87.4 \\
Perú & 2007 & 2538 & 2.7 & 3.8 & 10.7 & 0.09 & 109.5 \\
Perú & 2008 & 2538 & 2.8 & 3.7 & 6.0 & 0.03 & 59.7 \\
Perú & 2009 & 2538 & 2.8 & 3.5 & 6.4 & 0.05 & 69.8 \\
Perú & 2010 & 2538 & 2.9 & 3.2 & 4.3 & 0.12 & 41.9 \\
Nicaragua & 1998 & 1921 & 0.7 & 1.1 & 7.2 & 0.0 & 20.6 \\
Nicaragua & 2001 & 1921 & 1.0 & 1.4 & 9.3 & 0.0 & 34.1 \\
Nicaragua & 2005 & 1921 & 1.3 & 2.9 & 26.0 & 0.0 & 107.5 \\
Chile & 1996 & 3662 & 2.2 & 2.6 & 7.8 & 0.00 & 64.7 \\
Chile & 2001 & 3662 & 2.4 & 2.8 & 8.4 & 0.00 & 74.6 \\
Chile & 2006 & 3662 & 2.8 & 2.8 & 6.0 & 0.00 & 55.4 \\
\hline
\end{tabular}

Fuentes: Elaboración propia a partir de datos de las encuestas EPH (Argentina), ENAHO (Perú), CASEN (Chile) y ENMV (Nicaragua).

\subsection{Discusión de resultados}

\subsubsection{Argentina}

Después de la profunda crisis que enfrentó en el año 2002, Argentina ha transitado por un período de fuerte recuperación económica, exceptuando el año 2009 en que se vivieron las consecuencias de la crisis financiera de Estados Unidos. De acuerdo con cifras de la CEPAL (2012), el PIB por habitante medido en dólares de EEUU del 2005, pasó de 4,054.3 en el año 2003 a 6,784.3 en el 2011, con un incremento anual promedio de 6.8\%. Mientras que el ingreso por habitante medido en las mismas unidades se incrementó aún más, pasando de 3,821.8 dólares en el 2003 a 6,914.6 en el 2011, con un crecimiento promedio anual de $8.0 \%$. Estas cifras positivas también estuvieron acompañadas de importantes reducciones en el desempleo y en la pobreza. Según CEPAL (2012), la tasa de desempleo se redujo de $17.3 \%$ a $7.2 \%$ entre el año 2003 y el 2011. Mientras que según cálculos del 
CEDLAS, la tasa de incidencia de la pobreza con un umbral de 4 dólares diarios del año 2005, pasó de $36.6 \%$ en el segundo trimestre del año 2003 a $12.9 \%$ en el primer trimestre del año 2011.

En la figura 3.1 se presenta el panorama de la evolución reciente de las tasas de incidencia de la pobreza y de la vulnerabilidad en Argentina, según las estimaciones obtenidas en los resultados de este estudio. El gráfico muestra que tanto la pobreza como la vulnerabilidad experimentaron reducciones importantes durante el período. La reducción de la vulnerabilidad a la pobreza se presenta más acentuada en el componente de vulnerabilidad severa.

Figura 3.1: Evolución de la pobreza y la vulnerabilidad, Argentina (2003-2011).

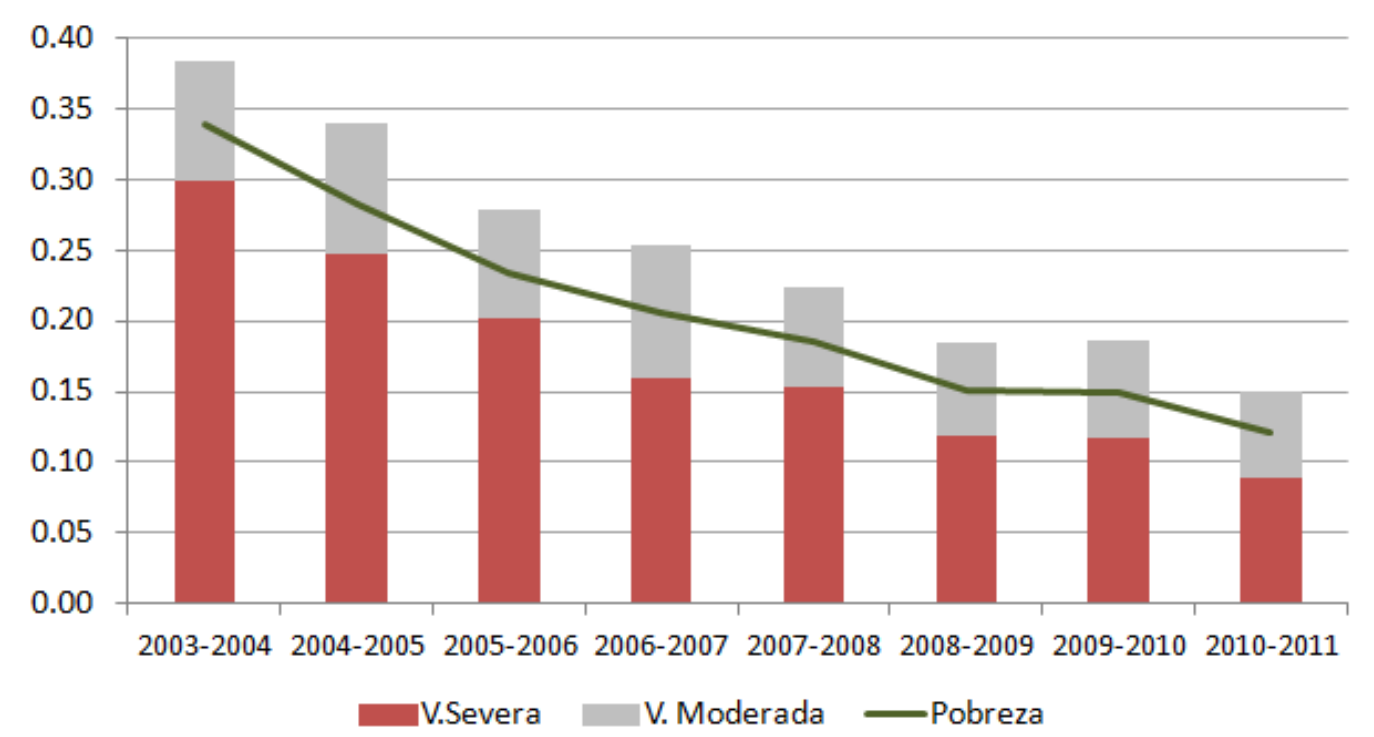

Fuente: Elaboración propia a partir de datos de paneles de la encuesta EPH.

En la tabla 3.2 se ofrecen las cifras detalladas de los índices estimados. Como puede observarse, los errores estándares de los índices de vulnerabilidad son pequeños y muy similares en magnitud a los errores de los índices de pobreza. ${ }^{12}$ Además dan cuenta de que la disminución en la incidencia de la vulnerabilidad a la pobreza, tanto agregada como en sus componentes severo y moderado, es estadísticamente significativa al $5 \%$ tanto a lo largo del período, como año a año, excepto entre los períodos 2008-2009 y 2009-2010, en los que Argentina sufrió los embates de la crisis económica mundial y los efectos de una sequía y una serie de protestas de los productores agropecuarios.

\footnotetext{
${ }^{12}$ Los errores estándares de los índices de vulnerabilidad fueron calculados usando técnicas de remuestreo, con 2,000 repeticiones. Ver detalle en el Anexo A.
} 
Tabla 3.2: Medidas FGT de Pobreza y Vulnerabilidad, Argentina (2003-2011).

\begin{tabular}{|c|c|c|c|c|c|c|c|c|}
\hline & $\begin{array}{l}2003 \\
2004\end{array}$ & 2004 & $\begin{array}{l}2005 \\
2006\end{array}$ & $\begin{array}{l}2006 \\
2007\end{array}$ & $\begin{array}{l}2007 \\
2008\end{array}$ & $\begin{array}{l}2008 \\
2009\end{array}$ & $\begin{array}{l}2009 \\
2010\end{array}$ & $\begin{array}{l}2010 \\
2011\end{array}$ \\
\hline \multicolumn{9}{|c|}{ Pobreza } \\
\hline$P_{0}$ & $\begin{array}{c}0.339 \\
(0.0051)\end{array}$ & $\begin{array}{c}0.282 \\
(0.0026)\end{array}$ & $\begin{array}{c}0.234 \\
(0.0024)\end{array}$ & $\begin{array}{c}0.205 \\
(0.0042)\end{array}$ & $\begin{array}{c}0.186 \\
(0.0035)\end{array}$ & $\begin{array}{c}0.151 \\
(0.0018)\end{array}$ & $\begin{array}{c}0.149 \\
(0.002)\end{array}$ & $\begin{array}{c}0.121 \\
(0.0018)\end{array}$ \\
\hline$P_{1}$ & $\begin{array}{c}0.149 \\
(0.0027)\end{array}$ & $\begin{array}{c}0.123 \\
(0.0015)\end{array}$ & $\begin{array}{c}0.098 \\
(0.0012)\end{array}$ & $\begin{array}{c}0.082 \\
(0.0021)\end{array}$ & $\begin{array}{c}0.072 \\
(0.0017)\end{array}$ & $\begin{array}{c}0.060 \\
(0.0009)\end{array}$ & $\begin{array}{c}0.056 \\
(0.0008)\end{array}$ & $\begin{array}{c}0.045 \\
(0.0008)\end{array}$ \\
\hline$P_{2}$ & $\begin{array}{c}0.091 \\
(0.0021)\end{array}$ & $\begin{array}{c}0.075 \\
(0.001)\end{array}$ & $\begin{array}{c}0.057 \\
(0.0009)\end{array}$ & $\begin{array}{c}0.046 \\
(0.0014)\end{array}$ & $\begin{array}{c}0.040 \\
(0.0013)\end{array}$ & $\begin{array}{c}0.035 \\
(0.0006)\end{array}$ & $\begin{array}{c}0.031 \\
(0.0006)\end{array}$ & $\begin{array}{c}0.025 \\
(0.0006)\end{array}$ \\
\hline \multicolumn{9}{|c|}{ Vulnerabilidad } \\
\hline$V_{0}$ & $\begin{array}{c}0.384 \\
(0.0054)\end{array}$ & $\begin{array}{c}0.339 \\
(0.0031)\end{array}$ & $\begin{array}{c}0.279 \\
(0.0028)\end{array}$ & $\begin{array}{c}0.2527208 \\
(0.0069)\end{array}$ & $\begin{array}{c}0.223 \\
(0.0041)\end{array}$ & $\begin{array}{c}0.185 \\
(0.0022)\end{array}$ & $\begin{array}{c}0.186 \\
(0.0022)\end{array}$ & $\begin{array}{c}0.150 \\
(0.0021)\end{array}$ \\
\hline$V_{1}$ & $\begin{array}{c}0.172 \\
(0.0032)\end{array}$ & $\begin{array}{c}0.143 \\
(0.0018)\end{array}$ & $\begin{array}{c}0.114 \\
(0.0015)\end{array}$ & $\begin{array}{c}0.11202876 \\
(0.0047)\end{array}$ & $\begin{array}{c}0.085 \\
(0.0021)\end{array}$ & $\begin{array}{c}0.068 \\
(0.0012)\end{array}$ & $\begin{array}{c}0.064 \\
(0.0011)\end{array}$ & $\begin{array}{c}0.049 \\
(0.0011)\end{array}$ \\
\hline$V_{2}$ & $\begin{array}{c}0.104 \\
(0.0027)\end{array}$ & $\begin{array}{c}0.082 \\
(0.0015)\end{array}$ & $\begin{array}{c}0.063 \\
(0.0012)\end{array}$ & $\begin{array}{c}0.070 \\
(0.0042)\end{array}$ & $\begin{array}{c}0.045 \\
(0.0017)\end{array}$ & $\begin{array}{c}0.035 \\
(0.001)\end{array}$ & $\begin{array}{c}0.031 \\
(0.0009)\end{array}$ & $\begin{array}{c}0.024 \\
(0.0009)\end{array}$ \\
\hline \multicolumn{9}{|c|}{ V. Severa } \\
\hline$V_{0}^{S}$ & $\begin{array}{c}0.299 \\
(0.0053)\end{array}$ & $\begin{array}{c}0.248 \\
(0.0029)\end{array}$ & $\begin{array}{c}0.202 \\
(0.0027)\end{array}$ & $\begin{array}{c}0.16010883 \\
(0.0062)\end{array}$ & $\begin{array}{c}0.152 \\
(0.0038)\end{array}$ & $\begin{array}{c}0.119 \\
(0.0021)\end{array}$ & $\begin{array}{c}0.117 \\
(0.002)\end{array}$ & $\begin{array}{c}0.088 \\
(0.0019)\end{array}$ \\
\hline$V_{1}^{S}$ & $\begin{array}{c}0.160 \\
(0.0032)\end{array}$ & $\begin{array}{c}0.128 \\
(0.0017)\end{array}$ & $\begin{array}{c}0.102 \\
(0.0015)\end{array}$ & $\begin{array}{c}0.0888375 \\
(0.0026)\end{array}$ & $\begin{array}{c}0.073 \\
(0.002)\end{array}$ & $\begin{array}{c}0.057 \\
(0.0011)\end{array}$ & $\begin{array}{c}0.053 \\
(0.001)\end{array}$ & $\begin{array}{c}0.040 \\
(0.001)\end{array}$ \\
\hline$V_{2}^{S}$ & $\begin{array}{c}0.100 \\
(0.0026)\end{array}$ & $\begin{array}{c}0.077 \\
(0.0014)\end{array}$ & $\begin{array}{c}0.059 \\
(0.0012)\end{array}$ & $\begin{array}{c}0.05743406 \\
(0.0019)\end{array}$ & $\begin{array}{c}0.042 \\
(0.0015)\end{array}$ & $\begin{array}{c}0.032 \\
(0.0009)\end{array}$ & $\begin{array}{c}0.028 \\
(0.0008)\end{array}$ & $\begin{array}{c}0.021 \\
(0.0008)\end{array}$ \\
\hline & oderada & & & & & & & \\
\hline$V_{0}^{M}$ & $\begin{array}{c}0.086 \\
(0.0033)\end{array}$ & $\begin{array}{c}0.091 \\
(0.0019)\end{array}$ & $\begin{array}{c}0.077 \\
(0.0017)\end{array}$ & $\begin{array}{c}0.093 \\
(0.0098)\end{array}$ & $\begin{array}{c}0.071 \\
(0.0025)\end{array}$ & $\begin{array}{c}0.065 \\
(0.0014)\end{array}$ & $\begin{array}{c}0.068 \\
(0.0015)\end{array}$ & $\begin{array}{c}0.061 \\
(0.0014)\end{array}$ \\
\hline$V_{1}^{M}$ & $\begin{array}{c}0.013 \\
(0.001)\end{array}$ & $\begin{array}{c}0.015 \\
(0.0006)\end{array}$ & $\begin{array}{c}0.012 \\
(0.0005)\end{array}$ & $\begin{array}{c}0.023 \\
(0.0046)\end{array}$ & $\begin{array}{c}0.011 \\
(0.0007)\end{array}$ & $\begin{array}{c}0.011 \\
(0.0005)\end{array}$ & $\begin{array}{c}0.011 \\
(0.0004)\end{array}$ & $\begin{array}{c}0.010 \\
(0.0004)\end{array}$ \\
\hline$V_{2}^{M}$ & $\begin{array}{c}0.004 \\
(0.0009)\end{array}$ & $\begin{array}{c}0.004 \\
(0.0006) \\
\end{array}$ & $\begin{array}{c}0.003 \\
(0.0004)\end{array}$ & $\begin{array}{c}0.012 \\
(0.0034) \\
\end{array}$ & $\begin{array}{c}0.003 \\
(0.0005) \\
\end{array}$ & $\begin{array}{c}0.003 \\
(0.0004) \\
\end{array}$ & $\begin{array}{c}0.003 \\
(0.0004) \\
\end{array}$ & $\begin{array}{c}0.003 \\
(0.0004) \\
\end{array}$ \\
\hline
\end{tabular}

Nota: Los subíndices corresponden a los valores de $\alpha$ para cada medida FGT. Los errores estándares en paréntesis fueron obtenidos por remuestreo con 2,000 repeticiones.

Fuente: Elaboración propia a partir de datos de paneles de la encuesta EPH.

Respecto de la vulnerabilidad moderada, aunque disminuye al considerar el período completo, dentro del período se comporta de manera oscilante, con un índice promedio de $7.6 \%$.

En cuanto a la composición entre la vulnerabilidad severa y la moderada, durante el período aumenta la importancia de la primera, ya que disminuye en una mayor proporción. La tasa de incidencia de la vulnerabilidad severa disminuye en un $70.4 \%$ durante el período, mientras que la tasa de incidencia de la vulnerabilidad moderada disminuye $28.2 \%$ en el mismo lapso. 
Cabe destacar que estos resultados dan cuenta también de una disminución en las brechas y en la profundidad de la pobreza y la vulnerabilidad. En el caso de la segunda, tal disminución está determinada por los cambios en la brecha y la profundidad del componente de primer momento. La profundidad de la vulnerabilidad severa experimenta un cambio muy importante, al pasar de 0.16 a 0.04 durante el período.

En la figura 3.2 se muestran las evoluciones de los índices de vulnerabilidad calculados junto con sus intervalos de confianza normales al 95\%, Vemos que la vulnerabilidad a la pobreza ha disminuido en el período de observación de acuerdo a todos los índices calculados.

Figura 3.2: Intervalos de confianza normales de los índices de vulnerabilidad, Argentina (20032011).
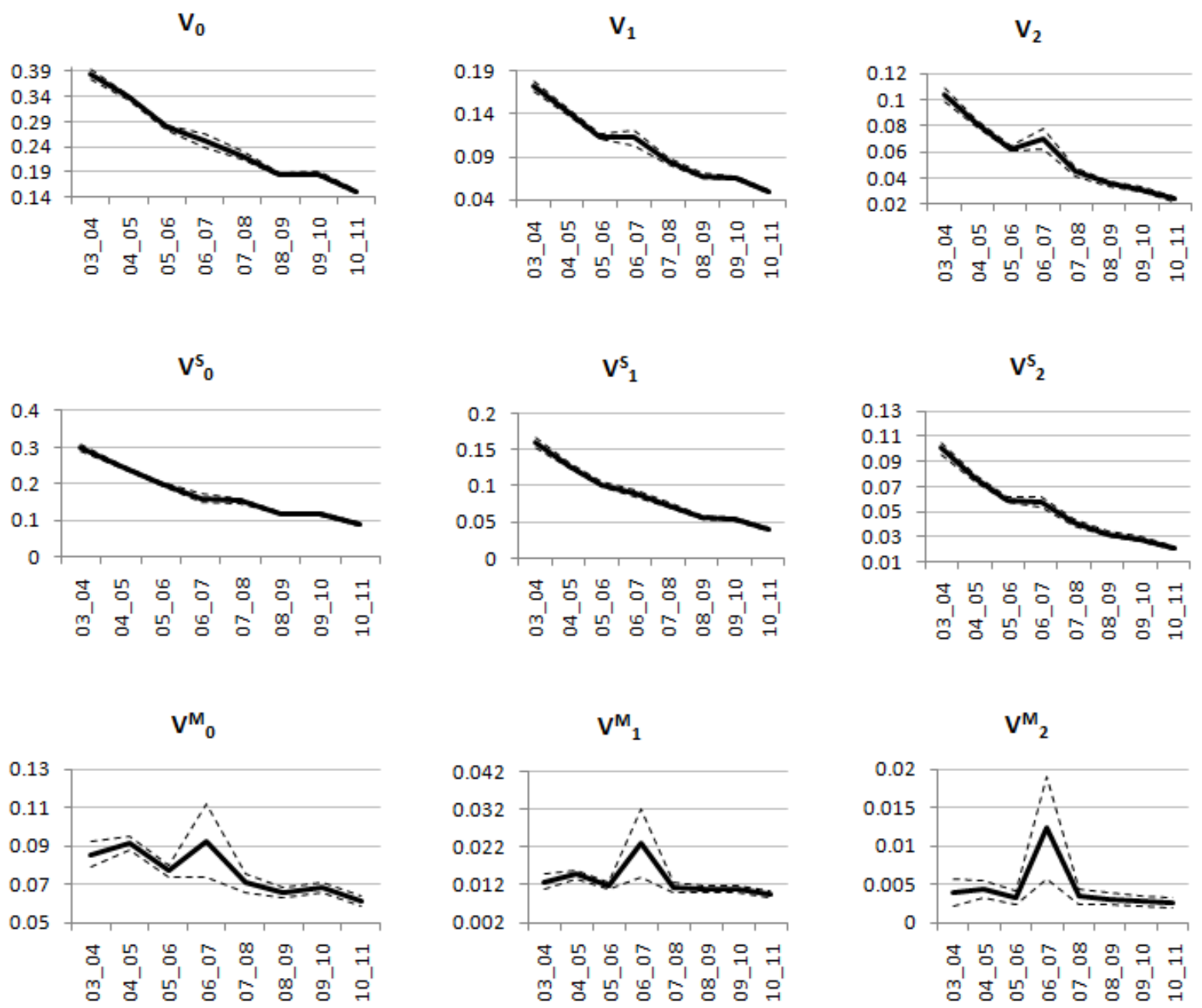

Fuente: Elaboración propia a partir de datos de paneles de la encuesta EPH.

Nota: Intervalos normales a un $95 \%$ de confianza, construidos por remuestreo con 2,000 repeticiones. 


\subsubsection{Perú}

En los últimos años Perú también ha gozado de una fase de mejoras en sus niveles promedios de bienestar. La economía creció sostenidamente entre los años 2004 y 2010, exceptuando el bache del año 2009 en que se sufrieron los efectos de la crisis financiera mundial.

Según cifras de CEPAL (2012), el PIB por habitante medido en dólares de EEUU del año 2005, pasó de 2,711.9 en el 2004 a 3,832.9 dólares en el año 2010, con un incremento anual promedio de $5.7 \%$. En tanto que el ingreso per cápita medido en las mismas unidades, pasó de 2,522.5 dólares en el 2004 a 3,791.7 en el 2010, con un incremento promedio anual de $6.5 \%$. La tasa de desempleo del país se redujo de 9.4 a $7.9 \%$ durante el período. Mientras que según cálculos del CEDLAS, la tasa de incidencia de la pobreza medida a 4 dólares diarios, pasó de $46.7 \%$ en el 2004, a 33.8\% en el 2010.

En la figura 3.3 se presenta la evolución de las tasas de incidencia de la pobreza y la vulnerabilidad en el Perú. Se observa una disminución en ambos índices durante el período.

Figura 3.3: Evolución de la pobreza y la vulnerabilidad, Perú (2004-2010).

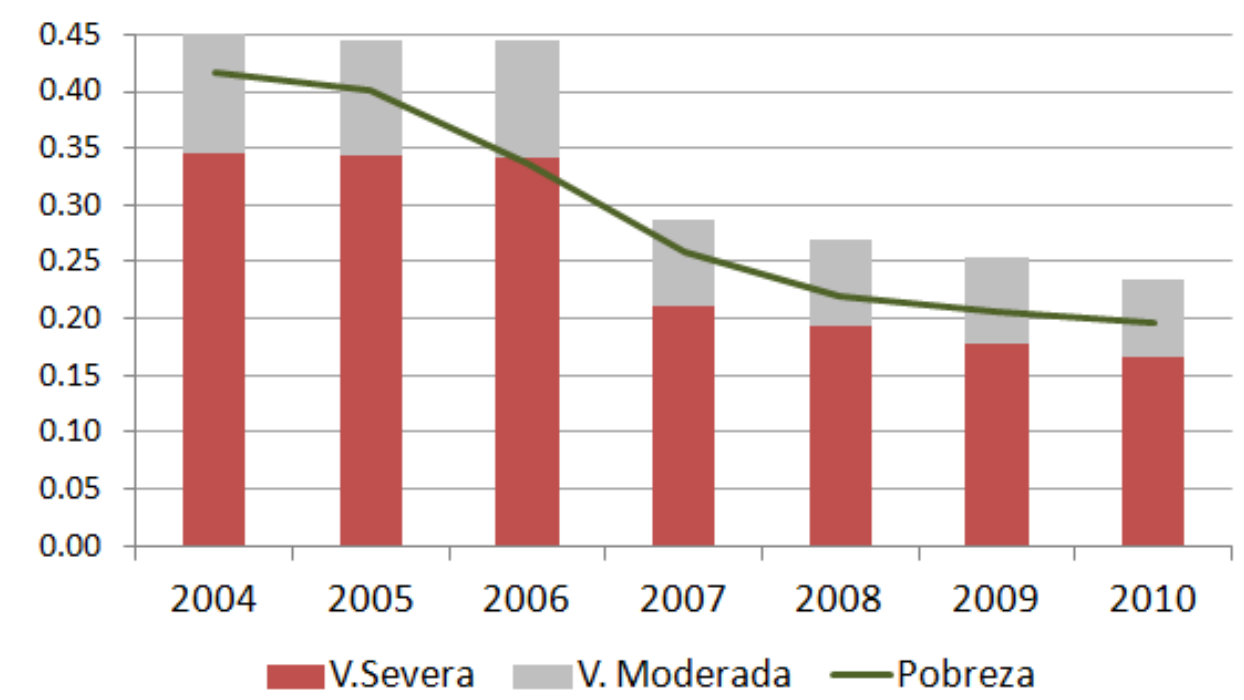

Fuente: Cálculos propios con datos de los paneles 2004-2006 y 2007-2010 de la encuesta de hogares ENAHO.

En relación a la cifra de pobreza, dicha disminución se ve más acentuada entre los años 2005 y 2007. Mientras que el indicador de vulnerabilidad denota un estancamiento en los tres primeros años y un salto entre los años 2006 y 2007. Sin embargo, tal salto puede 
deberse a diferencias en la selección de las muestras de ambos paneles o a un mayor sesgo de desgaste en el segundo panel de mayor longitud temporal. Las cifras de incidencia de pobreza en el Perú (a 4 dólares diarios), calculadas por el CEDLAS con datos de corte transversal, indican un descenso más paulatino en este indicador durante el período.

En la tabla 3.3, a continuación se presentan los cálculos detallados para cada índice de vulnerabilidad.

Tabla 3.3: Medidas FGT de Pobreza y Vulnerabilidad, Perú (2004-2010).

\begin{tabular}{|c|c|c|c|c|c|c|c|}
\hline & 2004 & 2005 & 2006 & 2007 & 2008 & 2009 & 2010 \\
\hline \multicolumn{8}{|c|}{ Pobreza } \\
\hline \multirow[t]{2}{*}{$P_{0}$} & 0.416 & 0.401 & 0.336 & 0.258 & 0.220 & 0.205 & 0.196 \\
\hline & $(0.0104)$ & $(0.0106)$ & $(0.0099)$ & $(0.0099)$ & $(0.0083)$ & $(0.0086)$ & $(0.0092)$ \\
\hline \multirow[t]{2}{*}{$P_{1}$} & 0.174 & 0.168 & 0.139 & 0.101 & 0.087 & 0.075 & 0.066 \\
\hline & $(0.0055)$ & $(0.0056)$ & $(0.0051)$ & $(0.0042)$ & $(0.0042)$ & $(0.0037)$ & $(0.0035)$ \\
\hline \multirow[t]{2}{*}{$P_{2}$} & 0.096 & 0.095 & 0.075 & 0.054 & 0.046 & 0.037 & 0.031 \\
\hline & $(0.0040)$ & $(0.0040)$ & $(0.0033)$ & $(0.0029)$ & $(0.0028)$ & $(0.0022)$ & $(0.0021)$ \\
\hline \multicolumn{8}{|c|}{ Vulnerabilidad } \\
\hline \multirow[t]{2}{*}{$V_{0}$} & 0.451 & 0.444 & 0.444 & 0.288 & 0.269 & 0.254 & 0.234 \\
\hline & $(0.0105)$ & $(0.0104)$ & $(0.0102)$ & $(0.011)$ & $(0.0103)$ & $(0.0098)$ & $(0.0099)$ \\
\hline \multirow[t]{2}{*}{$V_{1}$} & 0.188 & 0.187 & 0.186 & 0.118 & 0.101 & 0.088 & 0.077 \\
\hline & $(0.0057)$ & $(0.0054)$ & $(0.0052)$ & $(0.0054)$ & $(0.0046)$ & $(0.0041)$ & $(0.0043)$ \\
\hline \multirow[t]{2}{*}{$V_{2}$} & 0.103 & 0.102 & 0.102 & 0.063 & 0.050 & 0.041 & 0.034 \\
\hline & $(0.0041)$ & $(0.0038)$ & $(0.0037)$ & $(0.0038)$ & $(0.003)$ & $(0.0026)$ & $(0.003)$ \\
\hline \multicolumn{8}{|c|}{ Vulnerabilidad Severa } \\
\hline \multirow[t]{2}{*}{$V_{0}^{S}$} & 0.346 & 0.343 & 0.341 & 0.211 & 0.194 & 0.177 & 0.166 \\
\hline & $(0.0104)$ & $(0.0103)$ & $(0.0098)$ & $(0.0102)$ & $(0.0097)$ & $(0.0091)$ & $(0.009)$ \\
\hline \multirow[t]{2}{*}{$V_{1}^{S}$} & 0.174 & 0.173 & 0.173 & 0.106 & 0.091 & 0.078 & 0.069 \\
\hline & $(0.0057)$ & $(0.0055)$ & $(0.0052)$ & $(0.0053)$ & $(0.0045)$ & $(0.0041)$ & $(0.004)$ \\
\hline \multirow[t]{2}{*}{$V_{2}^{S}$} & 0.100 & 0.099 & 0.100 & 0.060 & 0.048 & 0.039 & 0.033 \\
\hline & $(0.0041)$ & $(0.0039)$ & $(0.0036)$ & $(0.0037)$ & $(0.0029)$ & $(0.0025)$ & $(0.0025)$ \\
\hline \multicolumn{8}{|c|}{ Vulnerabilidad Moderada } \\
\hline \multirow[t]{2}{*}{$V_{0}^{M}$} & 0.105 & 0.101 & 0.103 & 0.076 & 0.075 & 0.077 & 0.067 \\
\hline & $(0.0048)$ & $(0.0053)$ & $(0.0042)$ & $(0.0061)$ & $(0.0059)$ & $(0.0059)$ & $(0.0057)$ \\
\hline \multirow[t]{2}{*}{$V_{1}^{M}$} & 0.014 & 0.014 & 0.013 & 0.012 & 0.010 & 0.009 & 0.008 \\
\hline & $(0.0011)$ & $(0.0009)$ & $(0.0009)$ & $(0.0014)$ & $(0.0012)$ & $(0.0012)$ & $(0.0019)$ \\
\hline \multirow[t]{2}{*}{$V_{2}^{M}$} & 0.003 & 0.003 & 0.003 & 0.003 & 0.002 & 0.002 & 0.002 \\
\hline & $(0.0007)$ & $(0.0004)$ & $(0.0007)$ & $(0.001)$ & $(0.0008)$ & $(0.0009)$ & $(0.0017)$ \\
\hline
\end{tabular}

Nota: Los subíndices corresponden a los valores de $\alpha$ para cada medida FGT. Los errores estándares en paréntesis fueron obtenidos por remuestreo con 2,000 repeticiones.

Fuente: Cálculos propios con datos del panel de la encuesta de hogares ENAHO.

Los errores estándares para los estimadores de la vulnerabilidad en los paneles del Perú también son acotados. Vemos que en el primer panel (2004-2006) hay un estancamiento de la vulnerabilidad. Los cambios marginales que experimentan tales índices no 
son estadísticamente significativos. Para el segundo panel si se observa una caída significativa en la vulnerabilidad, que como puede notarse, está determinada principalmente por el descenso anual de la vulnerabilidad severa. La vulnerabilidad moderada también disminuye en el período cubierto por el segundo panel, pero en una proporción menor.

Cabe notar que en el caso del Perú también se observa una contracción en la profundidad de la pobreza y la vulnerabilidad durante el período, la que nuevamente está determinada por el componente de vulnerabilidad severa. En la figura 3.4 se presentan los intervalos de confianza para los indicadores calculados.

Figura 3.4: Intervalos de confianza normales de los índices de vulnerabilidad, Perú (2004-2010).
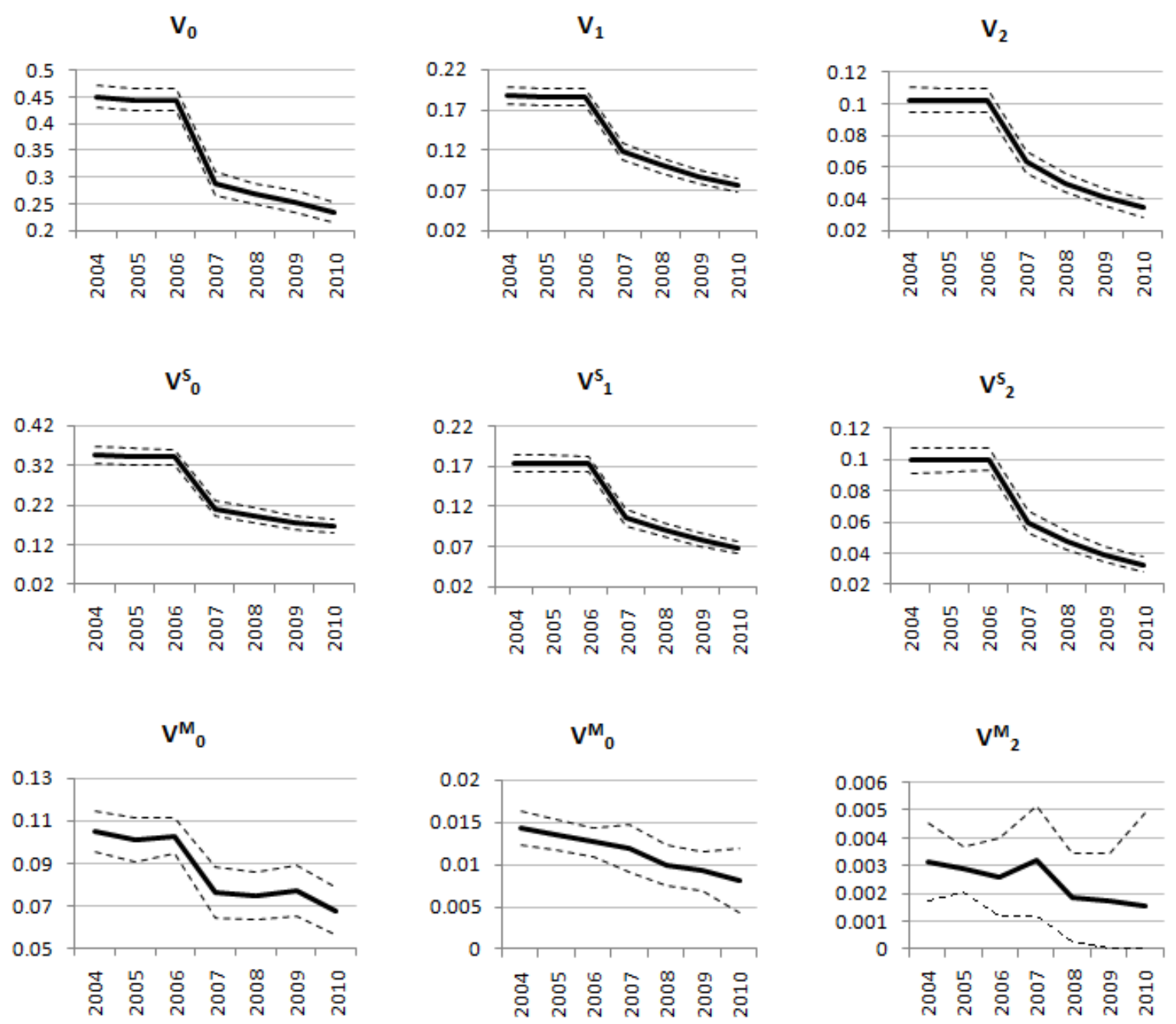

Fuente: Elaboración propia a partir de datos de paneles de la encuesta ENAHO.

Nota: Intervalos normales a un $95 \%$ de confianza, construidos por remuestreo con 2,000 repeticiones. 
Vemos que en el período cubierto por el primer panel (2004-2006) hay un estancamiento de la vulnerabilidad. El descenso de las medidas de vulnerabilidad no es significativo tal período, a pesar que la disminución de la pobreza si lo es (ver errores estándares de los índices de pobreza en la tabla 3.3).

\subsubsection{Nicaragua}

De acuerdo con cifras del Banco Central de Nicaragua, durante el período observado para el panel de este país, su PIB real creció a una tasa promedio anual de 3.8\%. Tal variación es apenas levemente superior al crecimiento anual promedio que ha experimentado esta economía durante los últimos 20 años (3.5\%). Sin embargo, según datos publicados por CEPAL (2012), durante el período se acumularon importantes avances en algunos indicadores sociales. El producto por habitante medido en dólares constantes de EEUU del año 2005, pasó de 984.3 en 1998 a 1,076.7 en el año 2001, y luego a 1,158.7 dólares en el 2005. Acumulando así una mejora de $17.7 \%$ durante el período. La tasa de desempleo experimentó reducciones importantes, pasando de $13.2 \%$ en el año 1998, a $11.3 \%$ en el 2001 y a $7.0 \%$ en el 2005. Por su parte, la tasa de incidencia de la pobreza medida a 4 dólares diarios también se redujo, pero a un ritmo moderado, manteniendo aún, niveles muy altos en comparación con otros países de América Latina. Según cálculos del CEDLAS con datos de muestras grandes de corte transversal, este indicador tomó valores de 69.8, 67.4 y $63.5 \%$ en los años 1998, 2001 y 2005 respectivamente.

Figura 3.5: Evolución de la pobreza y la vulnerabilidad, Nicaragua (1998-2005).

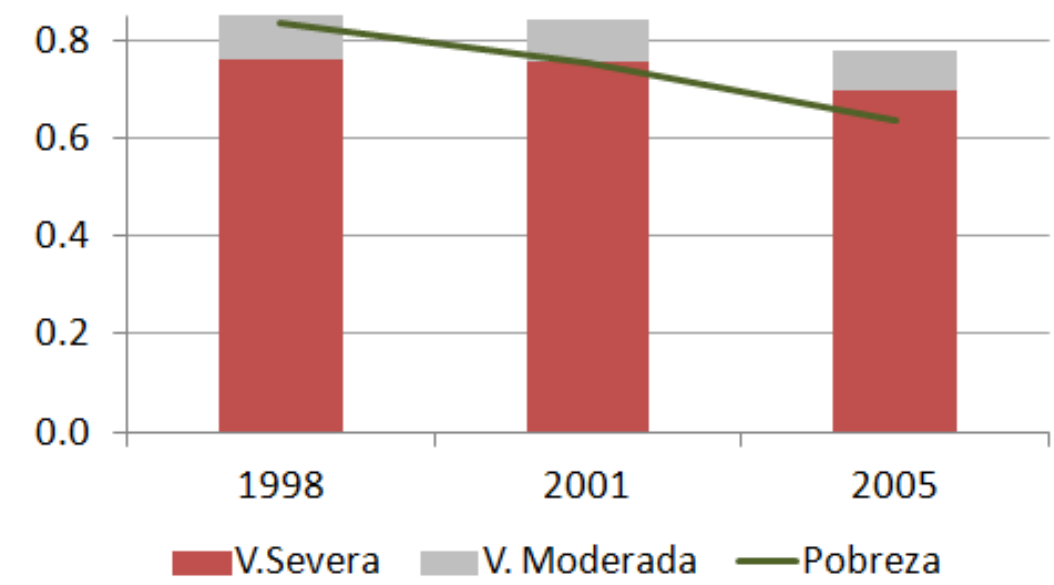

Fuente: Cálculos propios con datos del panel de la encuesta ENMV. 
En la figura 3.3 se muestra la evolución de los indicadores de pobreza y de vulnerabilidad para el panel de este país. Según ello, ambos indicadores disminuyen durante el período, pero la incidencia de la vulnerabilidad denota un estancamiento entre los años 1998 y 2001.

De todos los paneles analizados en este estudio, en el de Nicaragua es en donde se observa la mayor proporción de vulnerabilidad severa, lo cual es consistente con la segunda hipótesis planteada en la introducción. Se debe a que es el país más pobre de la muestra y el que posee una de las mayores tasas de incidencia de la pobreza en la región de América Latina y el Caribe. En la tabla 3.4, a continuación se ofrece el detalle de los índices calculados.

La disminución de la tasa de incidencia de la vulnerabilidad a la pobreza no es significativa entre 1998 y 2001. Ello posiblemente revelaría que los logros en disminución de la pobreza en el país avanzaron a un ritmo lento entre esos años. Pero sobre todo, es un indicio de que los períodos de disminución de la pobreza no siempre van acompañados de disminuciones en la vulnerabilidad a la pobreza. Por su parte, la incidencia de la vulnerabilidad severa tampoco disminuye entre esos años, ya que la caída de 0.76 a 0.75 en este componente, tampoco o es estadísticamente significativa al $5 \%$, según los errores estándares obtenidos. Tampoco la vulnerabilidad moderada tiene una caída significativa en tal período. La vulnerabilidad a la pobreza decrece en el país solo en el intervalo 2001-2005.

Sin embargo, en los resultados se observan progresos en los indicadores de brechas y de profundidad desde inicios del período de observación, tanto para la pobreza, como para la vulnerabilidad. Las reducciones en los indicadores de profundidad de la vulnerabilidad global y de la vulnerabilidad severa son significativas para el período completo, así como entre un período y otro. Es decir, aunque el porcentaje de vulnerables se mantiene entre los años 1998-2001, la intensidad de la vulnerabilidad empieza a decrecer desde ese entonces.

En la figura 3.6 se presentan los intervalos de confianza gaussianos, al 95\%, para los indicadores calculados. El dicha figura se observa más claramente tanto el estancamiento en la tasa de incidencia de la vulnerabilidad entre los años 1998-2001, como el descenso sostenido en la brecha y la profundidad de la vulnerabilidad a la pobreza desde inicios del período. 
Tabla 3.4: Medidas FGT de Pobreza y Vulnerabilidad, Nicaragua (1998-2005).

\begin{tabular}{|c|c|c|c|}
\hline & 1998 & 2001 & 2005 \\
\hline \multicolumn{4}{|c|}{ Pobreza } \\
\hline \multirow[t]{2}{*}{$P_{0}$} & 0.835 & 0.752 & 0.637 \\
\hline & $(0.010)$ & $(0.0140)$ & $(0.0127)$ \\
\hline \multirow[t]{2}{*}{$P_{1}$} & 0.485 & 0.399 & 0.285 \\
\hline & $(0.0089)$ & $(0.0087)$ & $(0.0081)$ \\
\hline \multirow[t]{2}{*}{$P_{2}$} & 0.334 & 0.255 & 0.163 \\
\hline & $(0.0082)$ & $(0.0078)$ & $(0.0054)$ \\
\hline \multicolumn{4}{|c|}{ Vulnerabilidad } \\
\hline \multirow[t]{2}{*}{$V_{0}$} & 0.851 & 0.840 & 0.776 \\
\hline & $(0.0106)$ & $(0.013)$ & $(0.0142)$ \\
\hline \multirow[t]{2}{*}{$V_{1}$} & 0.501 & 0.457 & 0.376 \\
\hline & $(0.0094)$ & $(0.0092)$ & $(0.0081)$ \\
\hline \multirow[t]{2}{*}{$V_{2}$} & 0.341 & 0.291 & 0.220 \\
\hline & $(0.0091)$ & $(0.0077)$ & $(0.006)$ \\
\hline \multicolumn{4}{|c|}{ Vulnerabilidad Severa } \\
\hline \multirow[t]{2}{*}{$V_{0}^{S}$} & 0.761 & 0.754 & 0.697 \\
\hline & $(0.0116)$ & $(0.0134)$ & $(0.0145)$ \\
\hline \multirow[t]{2}{*}{$V_{1}^{S}$} & 0.483 & 0.445 & 0.367 \\
\hline & $(0.0096)$ & $(0.0093)$ & $(0.0081)$ \\
\hline \multirow[t]{2}{*}{$V_{2}^{S}$} & 0.336 & 0.289 & 0.218 \\
\hline & $(0.0091)$ & $(0.0077)$ & $(0.006)$ \\
\hline \multicolumn{4}{|c|}{ Vulnerabilidad Moderada } \\
\hline \multirow[t]{2}{*}{$V_{0}^{M}$} & 0.089 & 0.086 & 0.080 \\
\hline & $(0.0067)$ & $(0.0078)$ & $(0.0058)$ \\
\hline \multirow[t]{2}{*}{$V_{1}^{M}$} & 0.018 & 0.012 & 0.008 \\
\hline & $(0.0021)$ & $(0.0016)$ & $(0.0007)$ \\
\hline \multirow[t]{2}{*}{$V_{2}^{M}$} & 0.005 & 0.002 & 0.001 \\
\hline & $(0.0016)$ & $(0.0009)$ & $(0.0004)$ \\
\hline
\end{tabular}

Nota: Los subíndices corresponden a los valores de $\alpha$ para cada medida FGT. Los errores estándares en paréntesis fueron obtenidos por remuestreo con 2,000 repeticiones.

Fuente: Cálculos propios con datos del panel de la encuesta ENMV.

\subsubsection{Chile}

Chile es el país de América Latina que ha exhibido los mayores avances económicos en décadas recientes. Durante el período de observación su PIB por habitante medido en dólares de EEUU del año 2005 (CEPAL, 2012) pasó de 6,052.2 en el año 1996, a 6,692.7 en el 2001 y luego a 7,882.4 dólares en el 2006, con un incremento acumulado de $30.2 \%$. Según cifras del CEDLAS, la tasa de incidencia de la pobreza del país, medida a 4 dólares diarios, era de $26.3 \%$ en el año 1996 y se redujo hasta $15.8 \%$ en el 2006.

En la figura 3.7 se presenta una visión de los resultados obtenidos para las tasas de 
Figura 3.6: Intervalos de confianza normales de los índices de vulnerabilidad, Nicaragua (19982006).
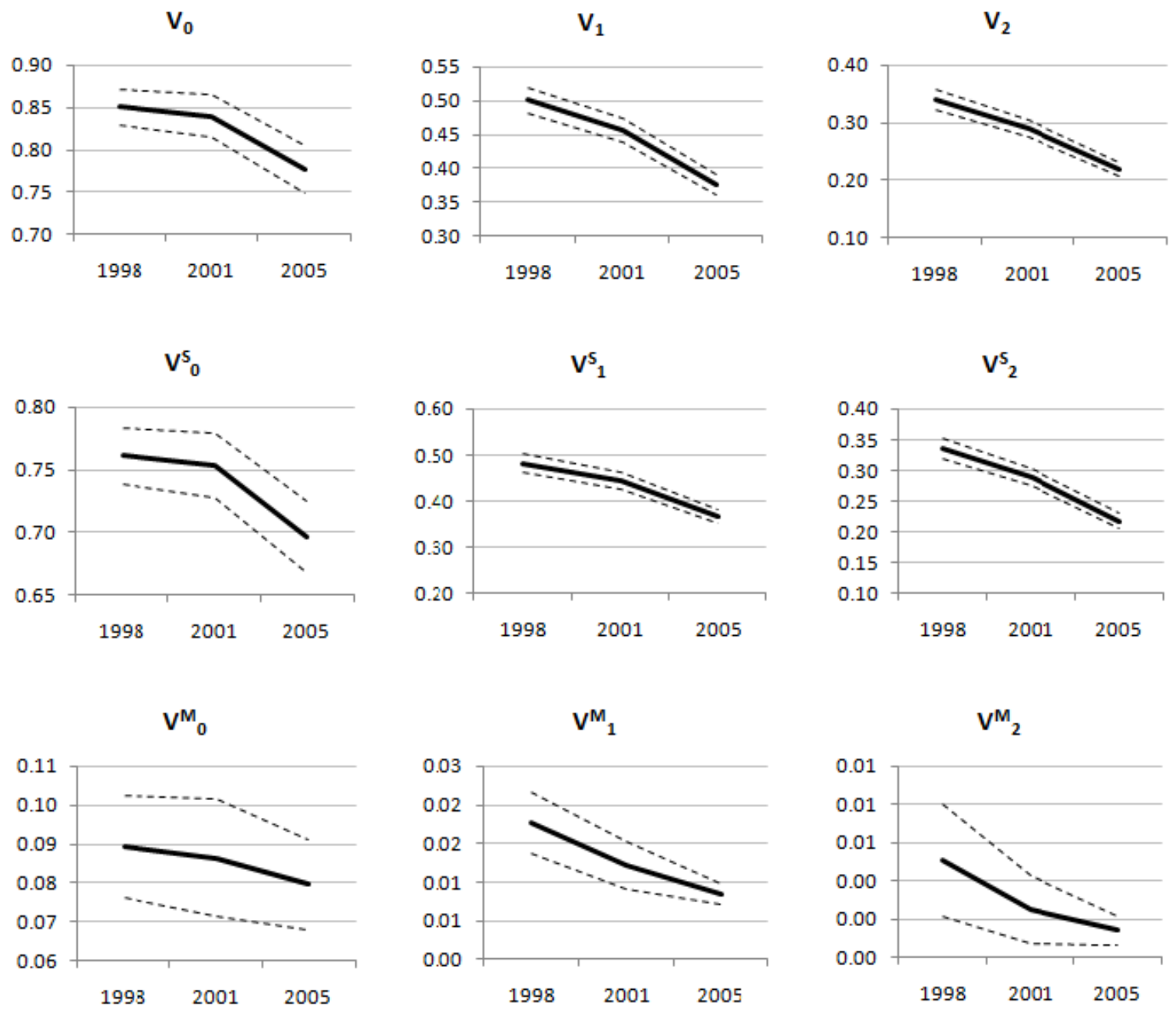

Fuente: Elaboración propia a partir de datos de paneles de la encuesta ENMV.

Nota: Intervalos normales a un $95 \%$ de confianza, construidos por remuestreo con 2,000 repeticiones.

incidencia de la pobreza y la vulnerabilidad en Chile.

Llama la atención el contraste de este gráfico con el anterior de Nicaragua (fig.3.3) en cuanto a la composición entre los dos tipos de vulnerabilidad. Al contrario de Nicaragua, Chile es el país de la muestra con menor incidencia de pobreza y con mayor proporción de vulnerabilidad moderada. En el gráfico se observa que la incidencia de la vulnerabilidad disminuye período a período, al igual que la tasa de incidencia de la pobreza.

En la tabla 3.5 se presentan el detalle de los indicadores obtenidos, mientras que la figura 3.13 se muestran los intervalos de confianza normales al $95 \%$ para los indicadores de vulnerabilidad. Durante el período, la tasa de incidencia de la vulnerabilidad en Chile 
Figura 3.7: Evolución de la pobreza y la vulnerabilidad, Chile (1996-2006).

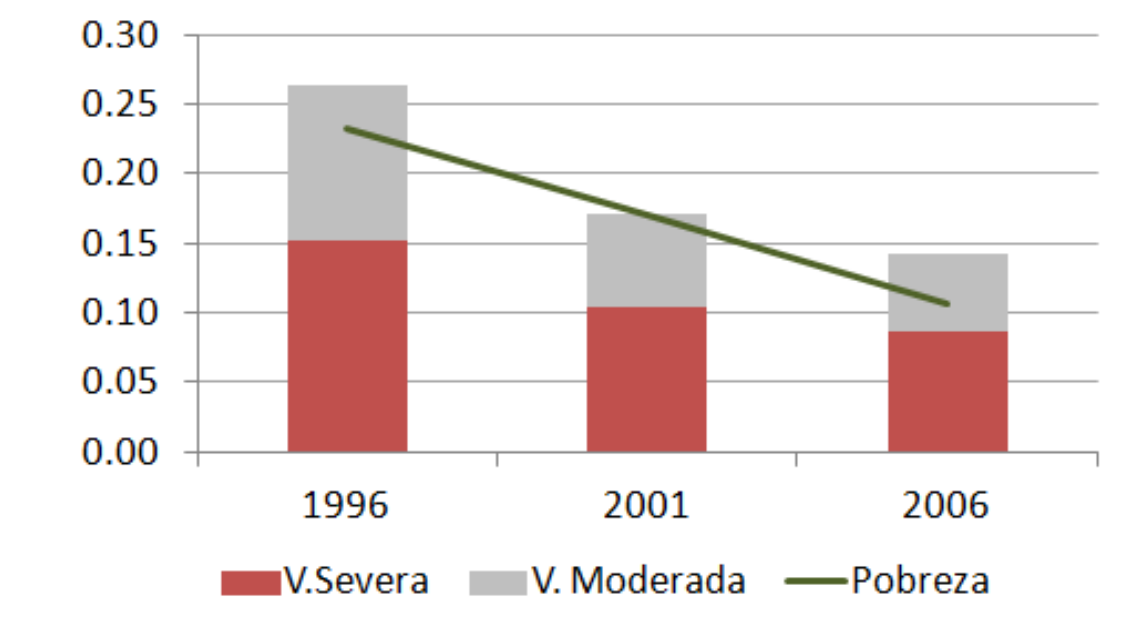

Fuente: Cálculos propios con datos del panel de la encuesta CASEN.

disminuye en forma significativa. Sin embargo, las medidas de brechas y de profundidad de la vulnerabilidad a la pobreza se han estancado entre los años 2001 y 2006. Es decir, aunque ha disminuido el porcentaje de hogares vulnerables, en términos estadísticos no se evidencian progresos en la intensidad de tal privación de bienestar. La profundidad de la vulnerabilidad a la pobreza se conserva en Chile entre los años 2001 y 2006. 
Tabla 3.5: Medidas FGT de Pobreza y Vulnerabilidad, Chile (1996-2006).

\begin{tabular}{|c|c|c|c|}
\hline & 1996 & 2001 & 2006 \\
\hline \multicolumn{4}{|c|}{ Pobreza } \\
\hline \multirow[t]{2}{*}{$P_{0}$} & 0.232 & 0.170 & 0.106 \\
\hline & $(0.0113)$ & $(0.0101)$ & $(0.0074)$ \\
\hline \multirow[t]{2}{*}{$P_{1}$} & 0.077 & 0.053 & 0.034 \\
\hline & $(0.0039)$ & $(0.0036)$ & $(0.0033)$ \\
\hline \multirow[t]{2}{*}{$P_{2}$} & 0.038 & 0.025 & 0.018 \\
\hline & $(0.0024)$ & $(0.0022)$ & $(0.0029)$ \\
\hline \multicolumn{4}{|c|}{ Vulnerabilidad } \\
\hline \multirow[t]{2}{*}{$V_{0}$} & 0.263 & 0.171 & 0.142 \\
\hline & $(0.0135)$ & $(0.0053)$ & $(0.0089)$ \\
\hline \multirow[t]{2}{*}{$V_{1}$} & 0.088 & 0.049 & 0.040 \\
\hline & $(0.0053)$ & $(0.0039)$ & $(0.0039)$ \\
\hline \multirow[t]{2}{*}{$V_{2}$} & 0.043 & 0.021 & 0.019 \\
\hline & $(0.0036)$ & $(0.0027)$ & $(0.0031)$ \\
\hline \multicolumn{4}{|c|}{ Vulnerabilidad Severa } \\
\hline \multirow[t]{2}{*}{$V_{0}^{S}$} & 0.152 & 0.105 & 0.086 \\
\hline & $(0.0125)$ & $(0.0099)$ & $(0.0081)$ \\
\hline \multirow[t]{2}{*}{$V_{1}^{S}$} & 0.068 & 0.041 & 0.034 \\
\hline & $(0.005)$ & $(0.0036)$ & $(0.0037)$ \\
\hline \multirow[t]{2}{*}{$V_{2}^{S}$} & 0.036 & 0.019 & 0.017 \\
\hline & $(0.0033)$ & $(0.0022)$ & $(0.0029)$ \\
\hline \multicolumn{4}{|c|}{ Vulnerabilidad Moderada } \\
\hline \multirow[t]{2}{*}{$V_{0}^{M}$} & 0.111 & 0.067 & 0.056 \\
\hline & $(0.0072)$ & $(0.0061)$ & $(0.0047)$ \\
\hline \multirow[t]{2}{*}{$V_{1}^{M}$} & 0.021 & 0.008 & 0.006 \\
\hline & $(0.0019)$ & $(0.0019)$ & $(0.0012)$ \\
\hline \multirow[t]{2}{*}{$V_{2}^{M}$} & 0.007 & 0.002 & 0.001 \\
\hline & $(0.0016)$ & $(0.0018)$ & $(0.0009)$ \\
\hline
\end{tabular}

Nota: Los subíndices corresponden a los valores de $\alpha$ para cada medida FGT. Los errores estándares en paréntesis fueron obtenidos por remuestreo con 2,000 repeticiones.

Fuente: Cálculos propios con datos del panel de la encuesta CASEN.

\subsubsection{Contrastes de las hipótesis planteadas}

En relación a la primera hipótesis planteada en la introducción, como ya se indicó en el análisis anterior, la incidencia de la vulnerabilidad a la pobreza disminuye en casi todos los países, para las muestras de paneles consideradas. Los intervalos de confianza normales al $95 \%$, calculados por remuestreo, indican que en los paneles observados se registran descensos de la vulnerabilidad que son estadísticamente significativos a un nivel de confianza de $95 \%$. Sin embargo, los resultados denotan también que existen períodos de estancamiento en los indicadores de vulnerabilidad a la pobreza para algunos países. 
Figura 3.8: Intervalos de confianza normales de los índices de vulnerabilidad, Chile (19962006).
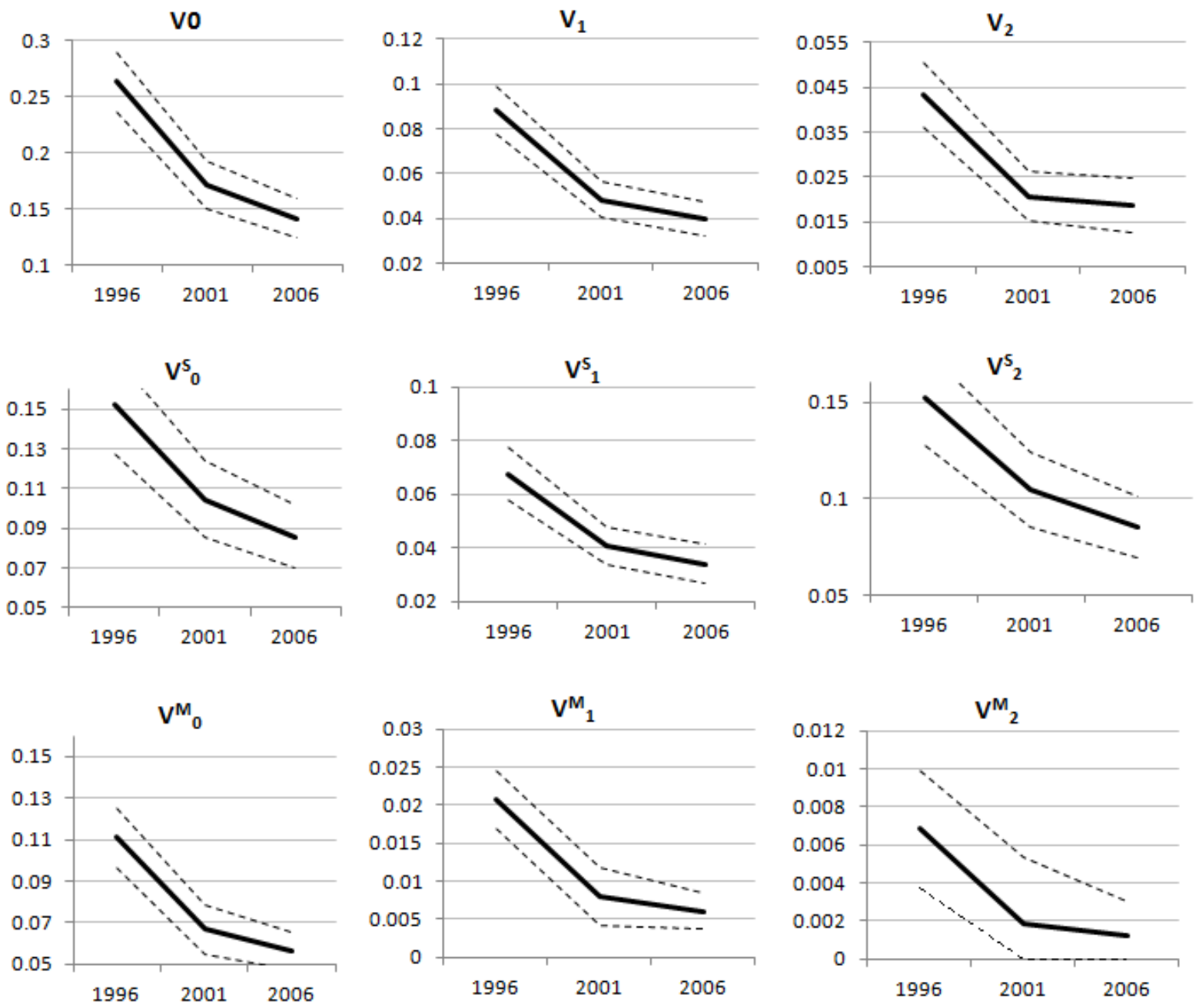

Fuente: Elaboración propia a partir de datos de paneles de la encuesta CASEN.

Nota: Intervalos normales a un $95 \%$ de confianza, construidos por remuestreo con 2,000 repeticiones.

En el caso del Perú, entre los años 2004 y 2006 se observa que la disminución de la pobreza no estuvo acompañada de descensos estadísticamente significativos en los niveles de vulnerabilidad (fig.3.4). Para el panel de Nicaragua se produce un estancamiento en la tasa de incidencia de la vulnerabilidad entre los años 1998 y 2001 (fig.3.6), aunque su intensidad disminuye en el mismo período. Para Chile en cambio, aunque la disminución de la tasa de incidencia de la pobreza ha ido acompañada de disminuciones en la tasa de incidencia de la vulnerabilidad, no ha sucedido lo mismo con la intensidad de esta última (fig.3.13). Esta evaluación no cambia si en lugar de contrastar la hipótesis con intervalos de confianza normales, se hace con intervalos de los percentiles (corregidos por sesgo), 
obtenidos también en el ejercicio de remuestreo. Como puede observarse en las figuras correspondientes que se presentan en el Anexo A, tales intervalos de confianza son muy similares a los normales y conducen a la mismas conclusiones.

En referencia a la segunda hipótesis, los contrastes estadísticos se realizaron tres contrastes estadísticos: una prueba ANOVA paramétrica con un factor fijo por rangos, una prueba ANOVA no paramétrica (Kruskal Wallis) y un contraste t-student por regresión simple. ${ }^{13} \mathrm{El}$ contraste no paramétrico se introdujo, porque a diferencia del contraste ANOVA habitual no exige que la variable respuesta se distribuya normal.

La variable respuesta en todos los contrastes es la proporción de la vulnerabilidad severa respecto de la tasa de incidencia de la vulnerabilidad total. El factor explicativo es la tasa de incidencia de la pobreza. Para proceder a ejecutar las dos primeras pruebas se generó un ranking con las 21 tasas de incidencia de la pobreza obtenidas en los cálculos previos y luego se separaron dichos indicadores en tres grupos iguales. El primer grupo corresponde las bajas tasas de incidencia de la pobreza (menores que 20\%), el segundo grupo a las tasas de incidencia media (que se sitúan entre $20 \%$ y $30 \%$ ) y el tercero corresponde a las tasas de incidencia alta (mayores que $30 \%$ ). La hipótesis nula en ambos casos es que no existen diferencias de medias de la variable respuesta entre los grupos. Los resultados de ambos contrastes estadísticos se presentan a continuación en las tablas 3.6 y 3.7 .

Tabla 3.6: Contraste ANOVA sobre la importancia de la vulnerabilidad severa.

\begin{tabular}{lccccc} 
& $\begin{array}{l}\text { Suma de } \\
\text { cuadrados }\end{array}$ & $\begin{array}{l}\text { Grados de } \\
\text { libertad }\end{array}$ & $\begin{array}{l}\text { Media } \\
\text { cuadrática }\end{array}$ & F & Valor p \\
\hline Inter-grupos & 0.129 & 2 & 0.065 & 19.239 & 0.000 \\
Intra-grupos & 0.060 & 18 & 0.003 & & \\
Total & 0.190 & 20 & & & \\
\hline
\end{tabular}

Fuente: Cálculos propios.

Vemos que la hipótesis nula se rechaza en ambos casos, lo cual aporta evidencia respecto de que la proporción entre la vulnerabilidad severa y la vulnerabilidad total está incidida por el nivel de la tasa de pobreza. Además, como lo observamos en el análisis anterior, tal relación es positiva. Ello se ve claramente en el siguiente gráfico en el cual introducimos además una línea de tendencia logarítmica.

\footnotetext{
${ }^{13}$ Este contraste estadístico equivale también a un ANOVA en donde el factor de agrupación corrresponde a la variable explicativa de la regresión. Téngase en cuenta que una variable aleatoria t-student al cuadrado con $g$ grados de libertad es equivalente una $\mathrm{F}$ de Fisher-Snedecor con un grado de libertad en el numerador y $g$ grados de libertad en el denominador.
} 
Tabla 3.7: Contraste Kruskal Wallis sobre la importancia de la vulnerabilidad severa.

\begin{tabular}{lc}
\hline Variable dependiente & $V_{0}^{S} / V_{0}$ \\
Variable de agrupación & Rango de $P_{0}$ \\
Chi-cuadrado & 14.716 \\
Grados de libertad & 2 \\
Valor p & 0.001 \\
\hline \multicolumn{2}{c}{ Fuente: Cálculos propios. }
\end{tabular}

Figura 3.9: Tasa de incidencia de la vulnerabilidad severa como proporción de la tasa de incidencia de la vulnerabilidad total. Relación con la tasa de incidencia de la pobreza.

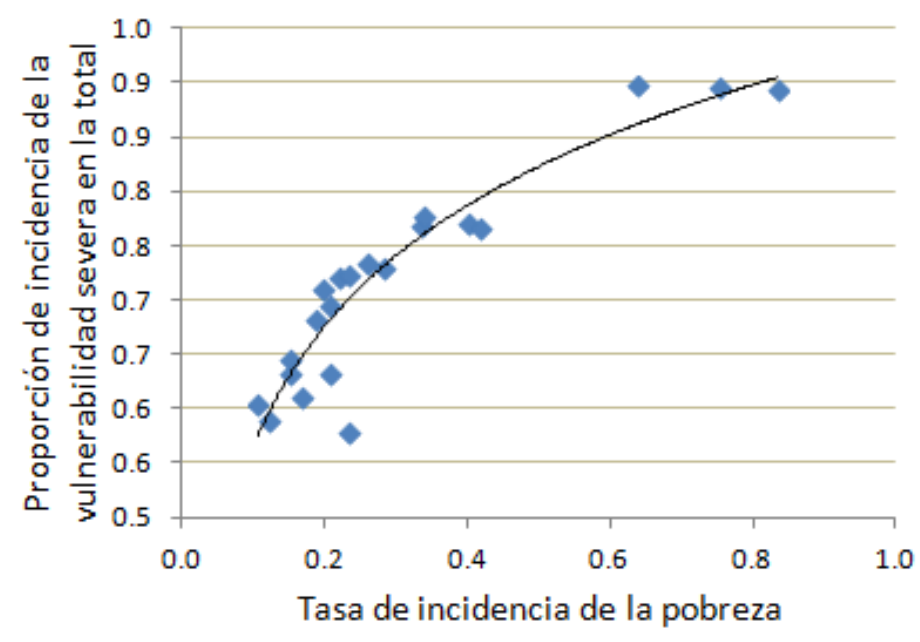

Fuente: Cálculos propios.

Finalmente, en la tabla 3.8 se presenta el resultado de la regresión simple correspondiente a la relación de la figura 3.9. El coeficiente asociado al logaritmo de la tasa de incidencia de la pobreza (l_pobreza) es positivo y estadísticamente significativo.

\subsubsection{Pruebas de robustez}

Para saber si las medidas presentadas en este capítulo son coherentes con la evolución de la vulnerabilidad a la pobreza que ofrecen otras medidas, se realizaron dos tipos de ejercicios de robustez. En primer lugar se les compara con indicadores del mimo tipo (Gallardo, 2013) para parámetros de aversión al riesgo distintos de uno. Se calcularon las tasa de incidencia de la vulnerabilidad a la pobreza de dicho indicador, para valores de $\gamma=0,5$ y $\gamma=0,75$.

En segundo lugar, se calcularon las tasas de incidencia de la vulnerabilidad a la pobreza 
Tabla 3.8: Regresión simple entre la participación de la vulnerabilidad severa en la tasa de incidencia de la vulnerabilidad total (share) y el logaritmo de la tasa de incidencia de la pobreza (1_pobreza).

\section{MCO, usando las observaciones 1-21 \\ Variable dependiente: share}

$\begin{array}{lllll} & \text { Coeficiente } & \text { Desv. Típica } & \text { Estadístico } t & \text { Valor p } \\ \text { const } & 0.933537 & 0.0214479 & 43.5257 & 0.0000 \\ \text { l_pobreza } & 0.160003 & 0.0147160 & 10.8727 & 0.0000\end{array}$

Media de la vble. dep.

0.717651 D.T. de la vble. dep.

0.097341

Suma de cuad. residuos

0.026240 D.T. de la regresión

0.037163

$R^{2}$

$0.861532 R^{2}$ corregido

0.854244

$F(1,19)$

118.2159 Valor p (de $F)$

$1.35 \mathrm{e}-09$

Log-verosimilitud

40.39465 Criterio de Akaike

$-76.78930$

Criterio de Schwarz

-74.70026 Hannan-Quinn

$-76.33592$

Fuente: Cálculos propios.

de acuerdo a los índices de Calvo y Dercon (2007b), Ligon y Schechter (2003) y Chaudhuri et al. $(2002) .{ }^{14}$

Finalmente, se calculó la vulnerabilidad media, medida por los índices de Calvo y Dercon (2007b) y de Ligon y Schechter (2003) para distintos parámetros de riesgo. En el caso de los indicadores de Calvo y Dercon (2007b), tales parámetros de riesgo corresponden a: $\rho=0,25, \rho=0,5$ y $\rho=0,75$. En el caso del indicador de Ligon y Schechter (2003), el indicador de calculó para una función de utilidad tipo CRRA (Aversión Relativa al Riesgo Constante), con parámetros: $\tau=1, \tau=2$ y $\tau=3$. Para el indicador de Chaudhuri et al.(2002) se usó la misma media estimada conforme la ecuación (3.7), en su versión logarítmica, mientras que la varianza se calculó simplemente con su análogo muestral tomando dicha media (sin corregir por un grado de libertad).

Para los paneles de Argentina (tabla 3.9) todos los indicadores de vulnerabilidad a la pobreza reportan una disminución de la vulnerabilidad en el período observado.

Para los indicadores de los paneles del Perú (tabla 3.10), también se observan resultados similares a los presentados antes. Tanto así, que tales indicadores también logran capturar un estancamiento en la vulnerabilidad a la pobreza en el panel 2004-2006.

\footnotetext{
${ }^{14}$ Para detalles sobre como se calculan estos indicadores, véase el capítulo 1.
} 
Tabla 3.9: Indicadores alternativos de vulnerabilidad, Argentina (2003-2011).

\begin{tabular}{lcccccccc}
\hline & 2003 & 2004 & 2005 & 2006 & 2007 & 2008 & 2009 & 2010 \\
& 2004 & 2005 & 2006 & 2007 & 2008 & 2009 & 2010 & 2011 \\
\hline \hline Índice de semidesviación típica & & & & & & & & \\
$\gamma=0.5$ & 0.335 & 0.289 & 0.235 & 0.199 & 0.185 & 0.149 & 0.149 & 0.113 \\
$\gamma=0,75$ & 0.360 & 0.313 & 0.256 & 0.222 & 0.201 & 0.165 & 0.166 & 0.129 \\
$\gamma=1$ & 0.384 & 0.339 & 0.279 & 0.253 & 0.223 & 0.185 & 0.186 & 0.150 \\
Índice de Clavo y Dercon & & & & & & & & \\
Tasa de incidencia & 0.299 & 0.248 & 0.202 & 0.160 & 0.152 & 0.119 & 0.117 & 0.088 \\
Vulnerabilidad media $(\rho=0,25)$ & 0.042 & 0.030 & 0.024 & 0.018 & 0.016 & 0.012 & 0.010 & 0.008 \\
Vulnerabilidad media $(\rho=0,5)$ & 0.074 & 0.054 & 0.043 & 0.032 & 0.029 & 0.022 & 0.019 & 0.014 \\
Vulnerabilidad media $(\rho=0,75)$ & 0.100 & 0.074 & 0.059 & 0.045 & 0.040 & 0.030 & 0.027 & 0.020 \\
Índice de Ligon y Shechter & & & & & & & & \\
Tasa de incidencia $(\tau=1)$ & 0.340 & 0.300 & 0.234 & 0.208 & 0.179 & 0.154 & 0.149 & 0.115 \\
Tasa de incidencia $(\tau=2)$ & 0.367 & 0.329 & 0.260 & 0.229 & 0.206 & 0.175 & 0.171 & 0.138 \\
Tasa de incidencia $(\tau=3)$ & 0.391 & 0.351 & 0.284 & 0.247 & 0.227 & 0.190 & 0.189 & 0.154 \\
Vulnerabilidad media en útiles $(\tau=1)$ & 0.279 & 0.226 & 0.159 & 0.127 & 0.110 & 0.097 & 0.082 & 0.067 \\
Vulnerabilidad media en útiles $(\tau=2)$ & 16.71 & 16.10 & 8.39 & 6.17 & 5.85 & 7.64 & 5.70 & 5.81 \\
Vulnerabilidad media en útiles $(\tau=3)$ & 8158.92 & 7903.02 & 4074.52 & 2983.26 & 2845.73 & 3757.50 & 2792.75 & 2861.18 \\
Índice de probabilidad de ser pobre & & & & & & & \\
Tasa de incidencia & 0.315 & 0.257 & 0.205 & 0.133 & 0.152 & 0.114 & 0.112 & 0.084 \\
\hline
\end{tabular}

Fuente: Cálculos propios con datos de paneles de la encuesta EPH.

Tabla 3.10: Indicadores alternativos de vulnerabilidad, Perú (2004-2010).

\begin{tabular}{lccccccc}
\hline & 2004 & 2005 & 2006 & 2007 & 2008 & 2009 & 2010 \\
\hline \hline Índice de semidesviación típica & & & & & & & \\
$\gamma=0,5$ & 0.393 & 0.392 & 0.389 & 0.245 & 0.230 & 0.215 & 0.194 \\
$\gamma=0,75$ & 0.422 & 0.416 & 0.416 & 0.265 & 0.252 & 0.231 & 0.215 \\
$\gamma=1$ & 0.416 & 0.401 & 0.336 & 0.288 & 0.269 & 0.254 & 0.234 \\
Índice de Clavo y Dercon & & & & & & & \\
Tasa de incidencia & 0.346 & 0.343 & 0.341 & 0.211 & 0.194 & 0.177 & 0.166 \\
Vulnerabilidad media $(\rho=0,25)$ & 0.043 & 0.044 & 0.044 & 0.023 & 0.020 & 0.017 & 0.014 \\
Vulnerabilidad media $(\rho=0,5)$ & 0.079 & 0.079 & 0.080 & 0.042 & 0.036 & 0.031 & 0.026 \\
Vulnerabilidad media $(\rho=0,75)$ & 0.108 & 0.109 & 0.110 & 0.058 & 0.050 & 0.043 & 0.037 \\
Índice de Ligon y Shechter & & & & & & & \\
Tasa de incidencia $(\tau=1)$ & 0.372 & 0.372 & 0.372 & 0.206 & 0.206 & 0.206 & 0.206 \\
Tasa de incidencia $(\tau=2)$ & 0.402 & 0.402 & 0.402 & 0.239 & 0.239 & 0.239 & 0.239 \\
Tasa de incidencia $(\tau=3)$ & 0.430 & 0.430 & 0.430 & 0.262 & 0.262 & 0.262 & 0.262 \\
Vulnerabilidad media en útiles $(\tau=1)$ & 0.217 & 0.217 & 0.217 & 0.093 & 0.093 & 0.093 & 0.093 \\
Vulnerabilidad media en útiles $(\tau=2)$ & 0.434 & 0.434 & 0.434 & 0.186 & 0.186 & 0.186 & 0.186 \\
Vulnerabilidad media en útiles $(\tau=3)$ & 1.191 & 1.191 & 1.191 & 0.539 & 0.539 & 0.539 & 0.539 \\
Índice de probabilidad de ser pobre & & & & & & & \\
& 0.361 & 0.360 & 0.355 & 0.214 & 0.197 & 0.185 & 0.172 \\
\hline
\end{tabular}

Fuente: Cálculos propios con datos de paneles de la encuesta ENAHO.

En el caso de Nicaragua (tabla 3.11), los indicadores alternativos también dan cuenta de una mayor disminución de la pobreza entre el año 2001 y el 2005. Mientras que entre los años 1998 y 2001 se observa también cierta persistencia de la vulnerabilidad.

Mientras tanto, para Chile (tabla 3.12), la vulnerabilidad disminuye en todos los in- 
dicadores, excepto por supuesto en el índice de Ligon y Schechter (2003) que no logra capturar tal dinámica, porque tal medida de vulnerabilidad corresponde al promedio del período. Ello confirma también lo reportado anteriormente acerca de la disminución de la tasa de incidencia de la vulnerabilidad a la pobreza en este país durante el período analizado.

Para el caso de Chile, Bérgolo (2012), también encuentran una disminución de la vulnerabilidad en el panel considerado.

Tabla 3.11: Indicadores alternativos de vulnerabilidad, Nicaragua (1998-2006).

\begin{tabular}{llll}
\hline & 1998 & 2001 & 2005 \\
\hline \hline Índice de semidesviación típica & & & \\
$\gamma=0,5$ & 0.816 & 0.800 & 0.737 \\
$\gamma=0,75$ & 0.836 & 0.822 & 0.763 \\
$\gamma=1$ & 0.851 & 0.840 & 0.776 \\
Índice de Clavo y Dercon & & & \\
Tasa de incidencia & 0.761 & 0.754 & 0.697 \\
Vulnerabilidad media $(\rho=0,25)$ & 0.140 & 0.129 & 0.104 \\
Vulnerabilidad media $(\rho=0,5)$ & 0.246 & 0.228 & 0.187 \\
Vulnerabilidad media $(\rho=0,75)$ & 0.327 & 0.306 & 0.254 \\
Índice de Ligon y Shechter & & & \\
Tasa de incidencia $(\tau=1)$ & 0.772 & 0.772 & 0.772 \\
Tasa de incidencia $(\tau=2)$ & 0.817 & 0.817 & 0.817 \\
Tasa de incidencia $(\tau=3)$ & 0.839 & 0.839 & 0.839 \\
Vulnerabilidad media en útiles $(\tau=1)$ & 0.626 & 0.626 & 0.626 \\
Vulnerabilidad media en útiles $(\tau=2)$ & 1.665 & 1.665 & 1.665 \\
Vulnerabilidad media en útiles $(\tau=3)$ & 10.18 & 10.18 & 10.18 \\
Índice de probabilidad de ser pobre & & & \\
Tasa de incidencia & 0.842 & 0.837 & 0.784 \\
\hline Fuente: Cálculos propios con datos de paneles de la encuesta ENMVI.
\end{tabular}


Tabla 3.12: Indicadores alternativos de vulnerabilidad, Chile (1996-2006).

\begin{tabular}{lccc}
\hline & 1996 & 2001 & 2006 \\
\hline \hline Índice de semidesviación típica & & & \\
$\gamma=0,5$ & 0.206 & 0.134 & 0.111 \\
$\gamma=0,75$ & 0.231 & 0.148 & 0.121 \\
$\gamma=1$ & 0.263 & 0.171 & 0.142 \\
Índice de Clavo y Dercon & & & \\
Tasa de incidencia & 0.152 & 0.105 & 0.086 \\
Vulnerabilidad media $(\rho=0,25)$ & 0.012 & 0.008 & 0.007 \\
Vulnerabilidad media $(\rho=0,5)$ & 0.022 & 0.014 & 0.013 \\
Vulnerabilidad media $(\rho=0,75)$ & 0.031 & 0.020 & 0.018 \\
Índice de Ligon y Shechter & & & \\
Tasa de incidencia $(\tau=1)$ & 0.126 & 0.126 & 0.126 \\
Tasa de incidencia $(\tau=2)$ & 0.157 & 0.157 & 0.157 \\
Tasa de incidencia $(\tau=3)$ & 0.193 & 0.193 & 0.193 \\
Vulnerabilidad media en útiles $(\tau=1)$ & 0.043 & 0.043 & 0.043 \\
Vulnerabilidad media en útiles $(\tau=2)$ & 0.147 & 0.147 & 0.147 \\
Vulnerabilidad media en útiles $(\tau=3)$ & 4.37 & 4.37 & 4.37 \\
Índice de probabilidad de ser pobre & & & \\
Tasa de incidencia & 0.125 & 0.080 & 0.065 \\
\hline Fuente: Cálculos propios con datos de paneles de la encuesta & CASEN.
\end{tabular}

\subsection{Conclusiones}

El índice de medición de la vulnerabilidad a la pobreza propuesto por Gallardo (2013) se ha sometido a prueba de validación empírica en este capítulo, utilizando 12 paneles de datos de encuestas de hogares para 4 países de América Latina. Los resultados obtenidos muestran que el indicador es informativo y refleja el riesgo que enfrentan los hogares de caer en pobreza o de permanecer en ella.

En el capítulo se ha evidenciado una ventaja importante de este índice de vulnerabilidad en comparación con otros propuestos en la literatura. Dicha ventaja consiste en que su interpretación es análoga a los índices de pobreza tipo FGT. Así, el índice permite comunicar respecto de la incidencia, la brecha y la profundidad de la vulnerabilidad a la pobreza, en forma similar a como se hace usualmente en los índices convencionales de pobreza.

En este estudio se muestra con una amplia gama de datos, que empíricamente es factible separar la vulnerabilidad severa explicada por la media, de la vulnerabilidad moderada, explicada por el riesgo asimétrico de caer bajo la media. Tal separación representa una contribución del índice de Gallardo (2013) para propósitos de analizar y compren- 
der mejor el fenómeno de la vulnerabilidad a la pobreza y para apoyar las decisiones de políticas públicas de manera informada.

Los resultados muestran que la incidencia de la vulnerabilidad a la pobreza ha disminuido en América Latina en casi todos los paneles de datos analizados. Con la excepción de algunos períodos, en donde se observan estancamientos en la evolución de la vulnerabilidad para algunos países, aún a pesar de que la pobreza se ha amenguado en ellos en el mismo lapso. Ello indica que no siempre la pobreza y la vulnerabilidad a la pobreza evolucionan al mismo ritmo.

Respecto del método de estimación, de acuerdo con los resultados obtenidos se ha mostrado que cumple su objetivo en forma satisfactoria. Ello valida la propuesta de Calvo (2008) respecto de la utilidad de un modelo con coeficientes específicos aleatorios en este contexto, en el que la media se estime alrededor de una tendencia individual.

Una contribución relevante de este trabajo es haber aportado evidencia de que a medida que los países avanzan en la disminución de la tasa de incidencia de la pobreza, disminuye la importancia relativa de la tasa de incidencia de la vulnerabilidad severa (determinada por la media) y aumenta la importancia relativa de la tasa de incidencia de la vulnerabilidad moderada (determinada por el riesgo). La utilidad práctica de este hallazgo empírico es orientadora en el siguiente sentido. Aquellos países con mayores tasas de pobreza deben concentrase principalmente en atacar las causas estructurales de esta privación y preocuparse por elevar los ingresos medios de la población. Mientras que los países con menores tasas de pobreza deben orientar esfuerzos en la generación de mecanismos de seguros para disminuir el riesgo de caer en pobreza por eventos aleatorios que desvían los resultados del bienestar de los hogares bajo su valor medio esperado.

\section{Referencias}

Amin, S., Rai A., Topa, G. (2003). "Does microcredit reach the poor and vulnerable? Evidence from Northern Bangladesh". Journal of Development Economics, 70(1): 59-82.

Andrews, D. y Buchinsky, M. (2000). "A Three-Step Method for Choosing the Number of Bootstrap Repetitions".Econometrica, 68 (1): pp. 23-51.

Arellano, M., Bover, O. (1990). "La Econometría de datos de panel". Investigaciones Económicas, 15(1): 3-45. 
Balestra, P. y Nerlove, M. (1966). "Pooling cross section and times series data in the estimation of a dynamic model: the demand for natural gas". Econometrica, 34: 585-612.

Bérgolo, M., Cruces G., Ham A. (2012). "Assessing the predictive power of vulnerability measures: evidence from panel data for Argentina and Chile". Journal of Economic Distribution, 21(1): 28-63.

Bourguignon, F. (2003). "The Growth Elasticity of Poverty Reduction". En el libro: Inequality and Growth, MIT Press, Cambridge. Editado por: Eicher T. y Turnovsky S..

Calvo, C., Dercon, S. (2005). "Measuring Individual Vulnerability". University of Oxford, Department of Economics Discussion Paper Series, No.229.

Calvo, C., Dercon, S. (2007a). "Chronic Poverty and All That: The Measurement of Poverty over Time". Centre for the Study of African Economies Series, WPS/2007-04.

Calvo, C., Dercon, S. (2007b). "Vulnerability to poverty". CSAE Working Paper 200703.

Calvo, C. (2008). "Vulnerability to Multidimensional Poverty: Peru, 1998-2002". World Development, 36(6): 1011-1020.

Cameron, C., Trivedi, P. (2009). Microeconometric using Stata. Stata Press.

CEPAL (2012). Panorama Social de América Latina. Santiago, Naciones Unidas.

Chaudhuri, S., Jalan, J., Suryahadi, A. (2002). "Assessing household vulnerability to poverty from cross-sectional data: a methodology and estimates from Indonesia". Department of Economics Discussion Paper Series, 0102-52, Columbia University.

Christiansen, L. y Subbarao, K. (2005). "Toward an understanding of vulnerability in rural Kenya". Journal of African Economies, 14(4): 540-558.

Davidson, A., y Hinkley, D. (1997). Bootstrap methods and their aplication. Cambridge University Press.

Deaton, A. (1997). The analysis of household surveys. A microeconometric approach to development policy. The Johns Hopkins University Press.

Dercon, S., Krishnan, P. (2000). "Vulnerability, seasonality and poverty in Ethiopia". Journal of Development Studies, 36(6), 25-53.

Dollar D. y Kray A. (2004). "Trade, Grow and Poverty". The Economic Journal, 114(493): F22-F49.

Dutta, I., J. Foster and A. Mishra (2011). " On measuring vulnerability to poverty". Social Choice and Welfare, 37(4): 743-761. 
Efron, B. y Tibshirani, R. (1993). An introduction to the bootstrap. Chapman and Hall, Inc.

Foster, J., Greer, J., Thorbecke, E. (1984). "A class of decomposable poverty measures". Econometrica, 52: 761-766.

Gallardo, M. (2013). "Using the downside mean-semideviation for measuring vulnerability to poverty". Economics Letters, 120(3): 416-418.

Gelman, A., Hill, J. (2007). Data Analysis Using Regression and Multilevel / Hierarchical Models. New York: Cambridge University Press.

Glewwe, P., Hall G. (1998). "Are some groups vulnerable to macroeconomic shocks than others? Hypothesis tests based on panel data from Peru", Journal of Development Economics, 56(1): 181-206.

Hsiao Ch. (2003). Analysis of Panel Data, Cambridge University Press.

Morduch, J. (1984). "Poverty and Vulnerability". The American Economic Review, 84(2): 221-225.

Ogryczak, W., Ruszczynski, A. (1999). "From stochastic dominance to mean-risk models: Semideviations as risk measures". European Journal of Operational Research, 116, 33-50.

Ogryczak, W., Ruszczynski, A. (2001). On consistency of stochastic dominance and mean-semideviations models. Mathematical Programming 89, 217-232.

Ligon, E., Schechter, L. (2003). "Measuring vulnerability". Economic Journal, 113(486): C95-C102.

Suryahadi, A., Sumarto, S. (2003). "Poverty and vulnerability in Indonesia before and after the economic crisis". Asian Economic Journal, 17(1), 45-64.

Swamy, P. (1970). "Efficient inference in a random coefficient regression model". Econometrica, 38(2), 311-323.

UBA (2010). "Informe técnico de la Universidad de Buenos Aires (UBA) con relación a la situación del INDEC". Disponible en: http://www.econ.uba.ar/www/institutos/eco nomia/Ceped/publicaciones/2010/informe \%20- \%20UBA.pdf.

Zellner, A. (1962). "An Efficient Method of Estimating Seemingly Unrelated Regressions and Tests for Aggregation Bias". Journal of the American Statistical Association, 57:348-368. 


\section{Anexo A}

Los errores estándares y los intervalos de confianza que se presentaron en este capítulo se calcularon por el procedimiento de remuestreo. Dicho procedimiento consistente en tomar $B$ sub-muestras aleatorias de cada panel y recalcular cada indicador con tales submuestras, obteniendo así $B$ indicadores aleatorios que permiten calcular el error estándar y los percentiles de la distribución generada en el ejercicio.

En la literatura no existe un consenso absoluto acerca del número de sub-muestras que se deben tomar para obtener un error estándar con una varianza aceptablemente pequeña. Algunos investigadores expertos en el tema (Efron y Tibshirani, 1993: 162; Davidson y Hinkley, 1997: 194) sugieren que un número de replicaciones entre 1,000 y 2,000 son suficientes para construir intervalos con coeficientes de confianza entre 90 y $95 \%$.

Por su parte, Andrews y Buchinsky (2000) propusieron elegir un valor de $B$, que depende de dos parámetros: $p d b$ y $\tau$. Donde $p d b$ es el porcentaje de desviación que estamos dispuesto a aceptar entre la medida de confiabilidad que queremos calcular $\lambda$ (el error estándar o el percentil deseado), del indicador de interés $\theta$, y el que obtendríamos con infinitas replicaciones. Mientras que $1-\tau$ es la probabilidad deseada para que dicho desvío no sea mayor que $p d b$. Es decir:

$$
\operatorname{Pr}\left(100 \frac{\left|\widehat{\lambda}_{B}-\widehat{\lambda}_{\infty}\right|}{\widehat{\lambda}_{\infty}} \leqslant p d b\right)=1-\tau
$$

En donde $\widehat{\lambda}_{B}$ es el error estándar o el percentil deseado que obtendríamos con $B$ replicaciones, mientras que $\widehat{\lambda}_{\infty}$ es el que obtendríamos con infinitas replicaciones.

El procedimiento de cálculo de $B$ propuesto Andrews y Buchinsky (2000) es iterativo en tres etapas, en las que se hacen correcciones de $B$ en dependencia de la asimetría de la distribución encontrada en el remuestreo. Sin embargo, para un $p d b=5 \%$ y $\tau=0,05$ el tamaño inicial de $B$ es 768 . Mientras que para un $p d b=5 \%$ y $\tau=0,01$, el $B$ inicial es 1326. Así que un número de replicaciones en el intervalo indicado por Efron y Tibshirani (1993: 162) y Davidson y Hinkley, (1997: 194), es suficientemente confiable para construir intervalos de confianza de un indicador.

Para esta investigación se eligió un $B=2,000$ para calcular dos tipos de intervalos de confianza: uno con distribución normal usando el error estándar obtenido en el remuestreo y otro tomando los percentiles de la distribución empírica obtenida en el mismo ejercicio. 
En el segundo caso se hizo un ajuste por sesgo, ya que el estimador con la muestra completa no necesariamente coincide con la media de la distribución empírica generada por las sub-muestras en el proceso de remuestreo. Las distribuciones obtenidas en el remuestreo son simétricas y normales, de manera que los intervalos construidos por el método percentil que se presentan a continuación, son muy similares a los obtenidos con distribuciones normales, ya presentados anteriormente.

Figura 3.10: Intervalos de confianza percentiles de los índices de vulnerabilidad corregidos por sesgo, Argentina (2003-2011).
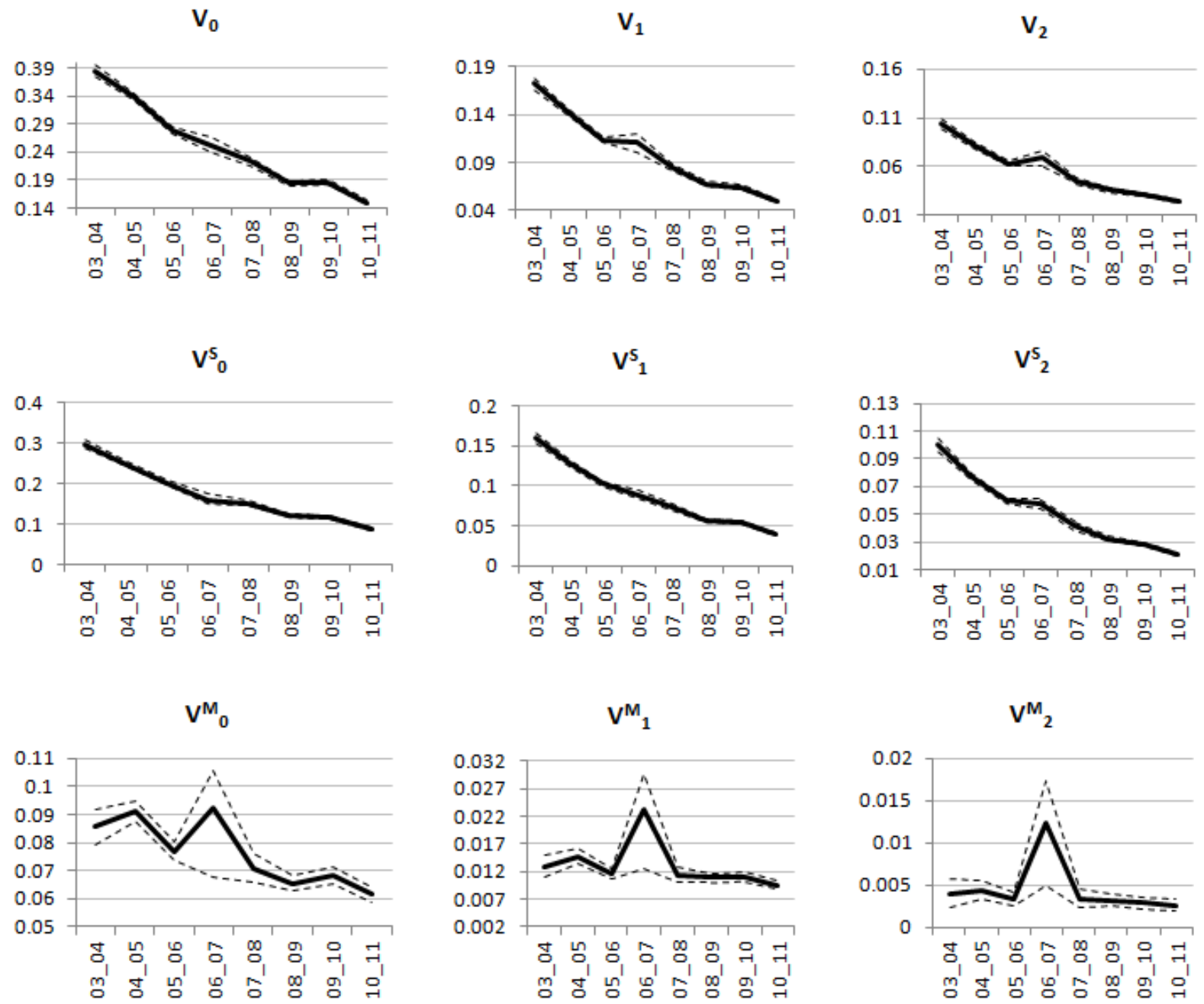

Fuente: Elaboración propia a partir de datos de paneles de la encuesta EPH.

Nota: Intervalos percentiles a un $95 \%$ de confianza, construidos por remuestreo con 2,000 repeticiones. 
Figura 3.11: Intervalos de confianza percentiles de los índices de vulnerabilidad corregidos por sesgo, Perú (2003-2010).
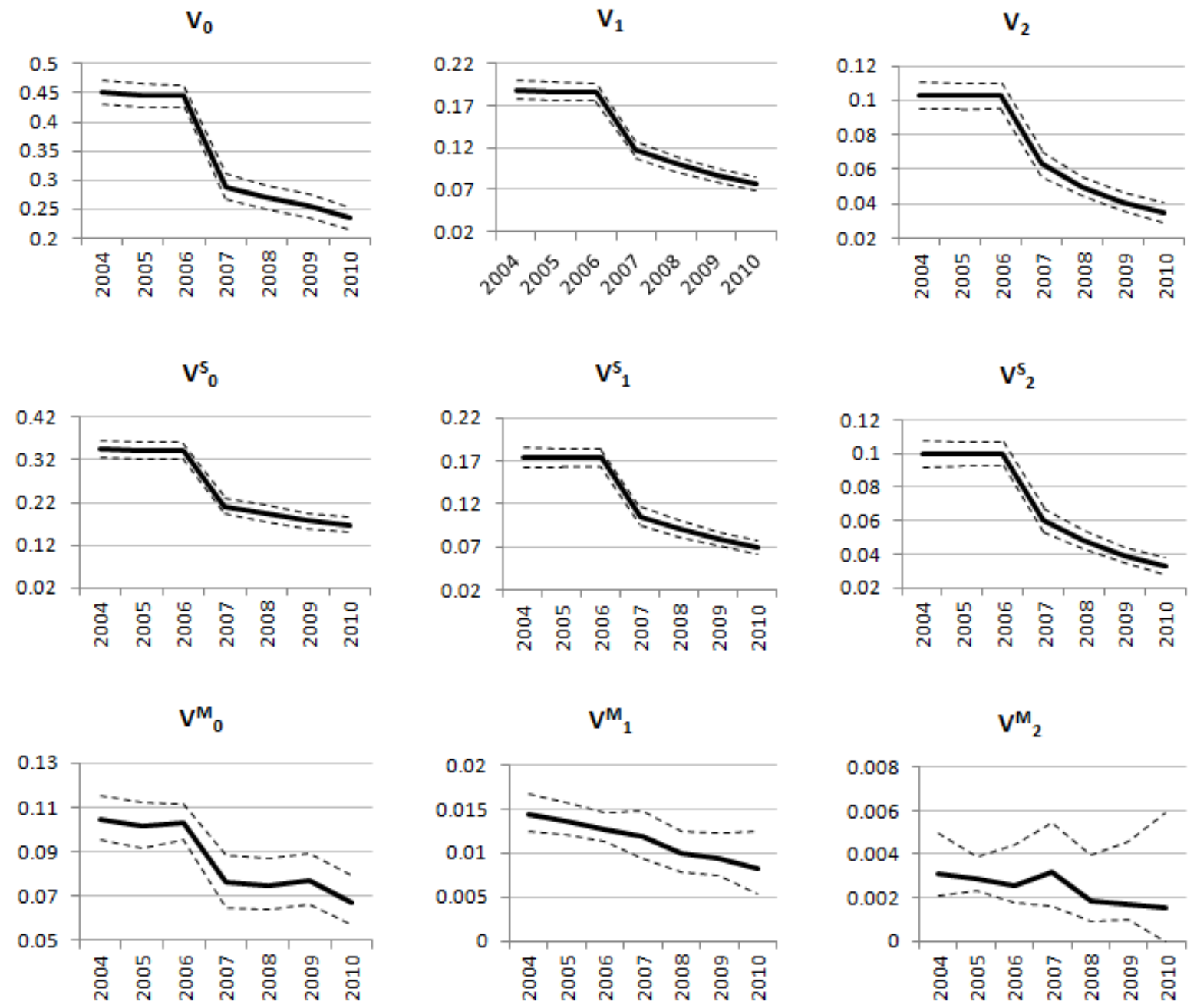

Fuente: Elaboración propia a partir de datos de paneles de la encuesta CASEN.

Nota: Intervalos percentiles a un $95 \%$ de confianza, construidos por remuestreo con 2,000 repeticiones. 
Figura 3.12: Intervalos de confianza percentiles de los índices de vulnerabilidad corregidos por sesgo, Nicaragua (1998-2005).
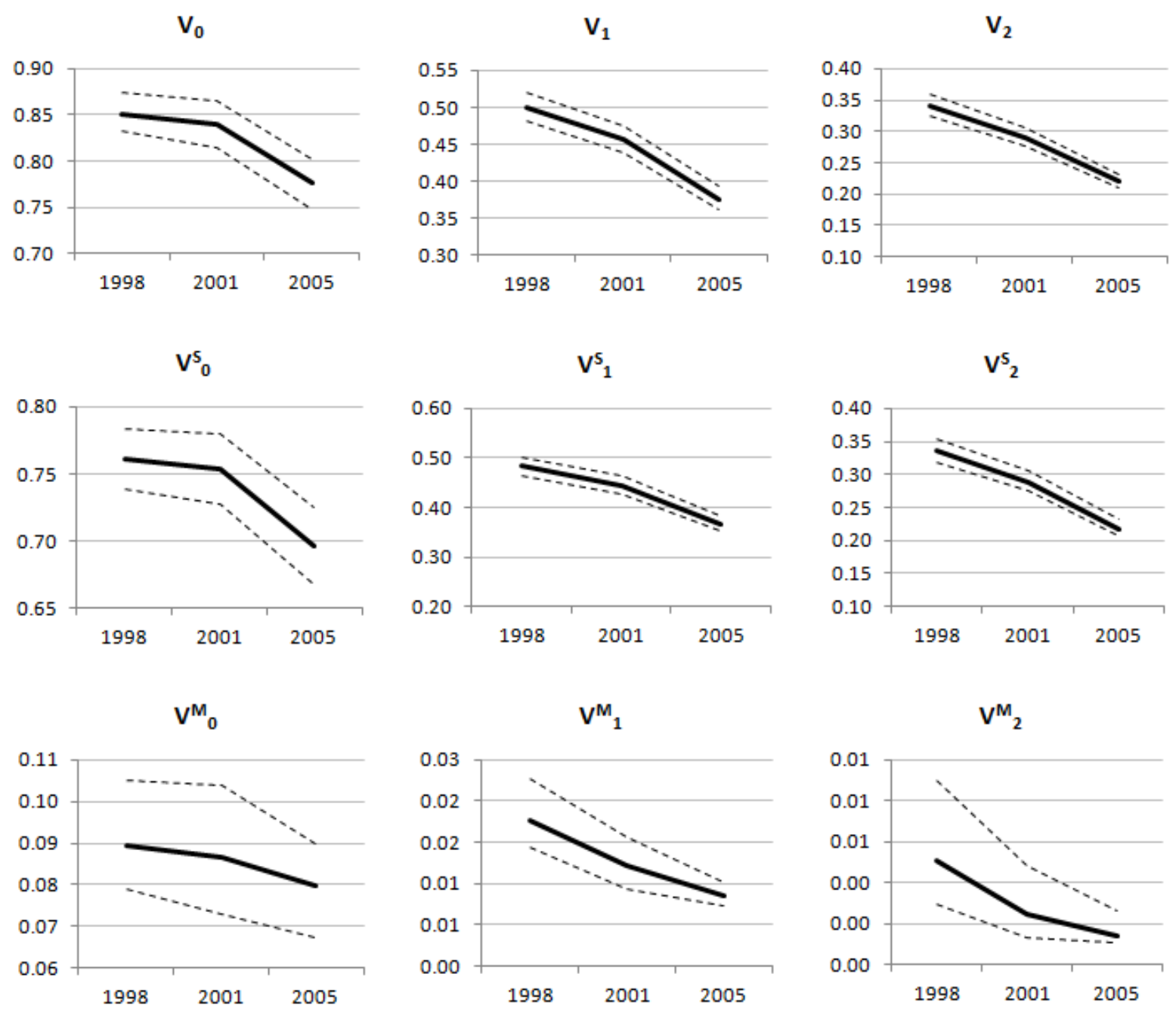

Fuente: Elaboración propia a partir de datos de paneles de la encuesta CASEN.

Nota: Intervalos percentiles a un $95 \%$ de confianza, construidos por remuestreo con 2,000 repeticiones. 
Figura 3.13: Intervalos de confianza percentiles de los índices de vulnerabilidad corregidos por sesgo, Chile (1996-2006).
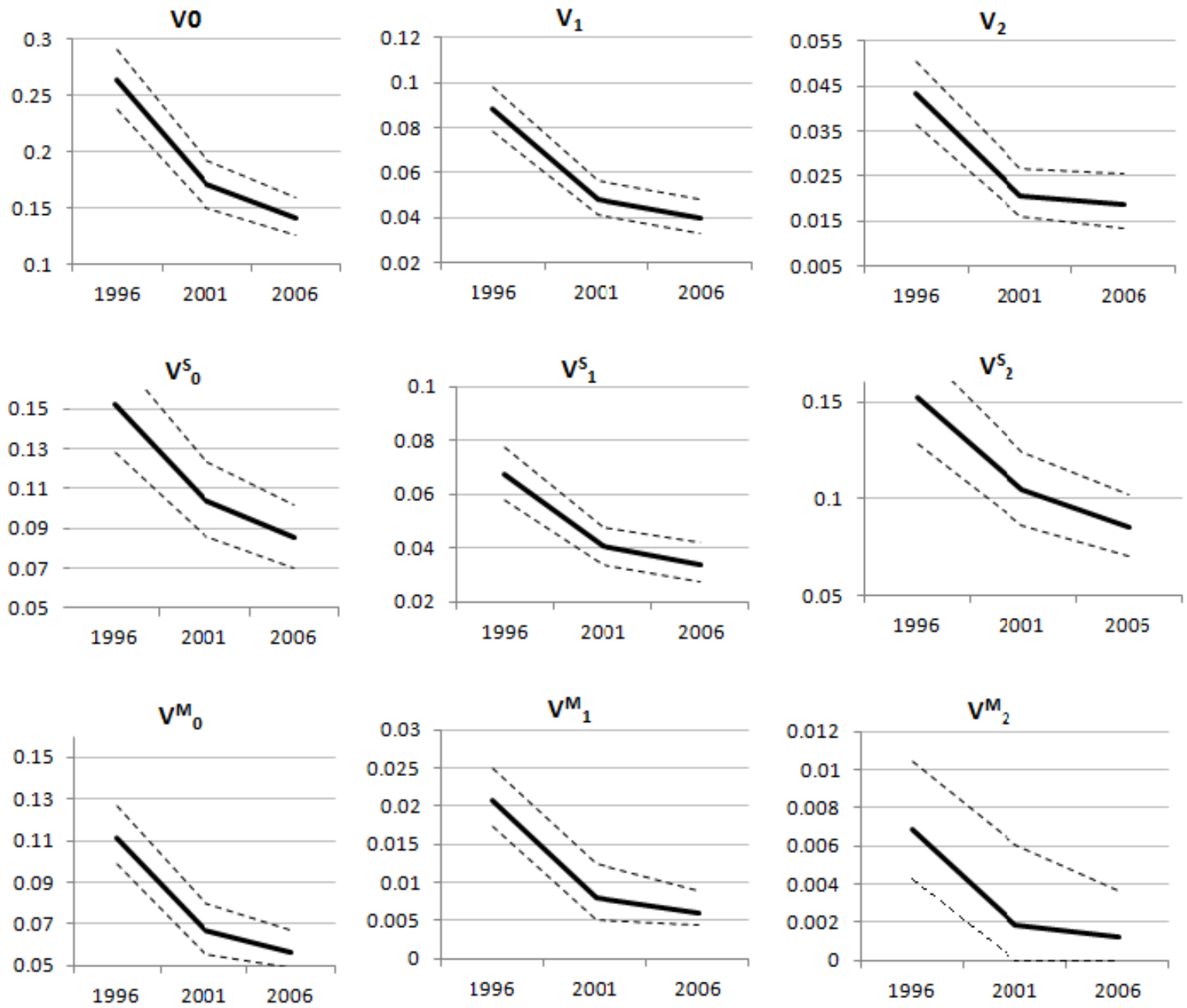

Fuente: Elaboración propia a partir de datos de paneles de la encuesta CASEN.

Nota: Intervalos percentiles a un $95 \%$ de confianza, construidos por remuestreo con 2,000 repeticiones. 
CAPÍTULO 3. EVIDENCIA EMPÍRICA PARA AMÉRICA LATINA 


\section{Conclusiones}

En el primer capítulo de esta tesis, se realiza una revisión crítica de veinte años de literatura sobre vulnerabilidad a la pobreza. Se discute el problema de la identificación de la vulnerabilidad a la pobreza en una población, a partir del estado del arte vigente hasta el año 2013.

Se revisan uno a uno, nueve enfoques distintos sobre vulnerabilidad a la pobreza, enfatizando en sus aportes a la conceptualización del fenómeno y en sus limitaciones.

Como resultado de tal revisión, se concluye que los dos elementos para identificar a los vulnerables a la pobreza dentro de una población son: tener un valor esperado del bienestar bajo la línea de pobreza, o estar en riesgo de caer en la pobreza, debido a las desviaciones del bienestar por debajo de dicho valor esperado.

Todos los enfoques de vulnerabilidad analizados incorporan uno de estos dos elementos, o ambos, en sus criterios de identificación. De acuerdo con ello, tales enfoques se pueden clasificar en dos vertientes: los que enfatizan en el elemento de exposición al riesgo y los que enfatizan en el elemento de pobreza esperada.

Se indican asimismo los nuevos planteamientos que extienden el terreno de la discusión hacia la vulnerabilidad a la pobreza relativa, la vulnerabilidad subjetiva a la pobreza y la vulnerabilidad a la pobreza multidimensional.

Las principales contribuciones de tal capítulo son: haber brindado un análisis del estado del arte sobre el concepto de vulnerabilidad a la pobreza y haber indicado los elementos claves para acercar las posiciones del debate en favor de ahondar en la comprensión del fenómeno.

El segundo capítulo constituye el núcleo central de la tesis y su principal contribución a la literatura. En dicho capítulo se introduce un nuevo enfoque de vulnerabilidad a la pobreza, sustentado en un modelo asimétrico de media-riesgo, que tiene sus raíces en la literatura de finanzas. 
Utilizando tal modelo, se estructura un ordenamiento racional de los resultados aleatorios del bienestar de los hogares, en un pre orden completo, mediante un criterio de dominancia en media-riesgo. El procedimiento de ordenación es similar a la comparación de retornos inciertos de los activos financieros y consistente con la dominancia estocástica de segundo orden. Tal ordenación permite identificar a los hogares vulnerables a la pobreza, al comparar los resultados aleatorios de su bienestar con la línea de pobreza bajo certidumbre.

Mediante el mismo enfoque de media-riesgo se consigue realizar una distinción nueva en la literatura, entre dos tipos de vulnerabilidad a la pobreza: la vulnerabilidad severa o de primer momento, vinculada a la pobreza esperada, y la vulnerabilidad moderada o de segundo momento, vinculada al riesgo de caer en pobreza debido a las desviaciones del bienestar bajo su valor esperado.

Para medir la vulnerabilidad a la pobreza de la población total se propone una nueva medida resumen, basada en un diseño tipo FGT, que utiliza la semi-desviación típica a la baja como parámetro de riesgo. El cálculo del índice agregado no requiere supuestos sobre las distribuciones de probabilidad de la variable focal, sino solamente estimaciones de sus medias muestrales y de sus semi-desviaciones típicas a la baja.

El diseño FGT del índice propuesto garantiza el cumplimiento de la base axiomática deseada, común a los indicadores de pobreza de esta clase. Lo cual se demuestra formalmente en el capítulo.

El cálculo del índice se ilustra en un ejemplo empírico, con datos de un panel balanceado de 2,561 hogares del Perú. En tal ejemplo se muestra que el cálculo del índice propuesto es simple y que este presenta un potencial muy prometedor para ser utilizado con propósitos de políticas públicas.

Una versión concisa del segundo capítulo de la tesis, ya fue publicada este año en la revista Economics Letters, bajo el título: "Using the downside mean-semideviation for measuring vulnerability to poverty". Con ello, el nuevo enfoque propuesto en esta tesis, pasó ya a formar parte de la literatura científica.

En el tercer capítulo el índice de medición propuesto se somete a un examen de validación empírica más riguroso, utilizando 12 paneles de datos de encuestas de hogares para 4 países de América Latina. Se encuentra evidencia robusta de que el indicador resiste ser utilizado en diversidad de escenarios. También se somete el indicador a un extensivo 
ejercicio de remuestreo con 2,000 replicaciones en 189 cálculos distintos. Se comprueba que su error estándar es pequeño. Los resultados obtenidos muestran que el indicador es informativo y que logra capturar en una medida resumen satisfactoria, el riesgo que enfrentan los hogares de caer en pobreza o de permanecer en ella.

En el mismo capítulo se logra evidenciar una ventaja importante del índice propuesto, en comparación con otros que existen en la literatura, con los cuales se realizan pruebas de robustez. Dicha ventaja consiste en que la interpretación del nuevo indicador es análoga a los índices de pobreza tipo FGT. Así, la nueva medida permite comunicar respecto de la incidencia, la brecha y la profundidad de la vulnerabilidad a la pobreza, en forma similar a como lo hacen los índices convencionales tipo FGT para la pobreza efectiva.

En tal capítulo se muestra con una amplia gama de datos, que empíricamente es factible separar la vulnerabilidad severa explicada por la media, de la vulnerabilidad moderada, explicada por el riesgo asimétrico de caer bajo la media. Tal separación permite analizar y comprender mejor el fenómeno de la vulnerabilidad a la pobreza y brinda información muy relevante para apoyar las decisiones de políticas públicas de manera informada.

En los resultados del mismo capítulo se aporta evidencia estadística en favor de la hipótesis de que la incidencia de la vulnerabilidad a la pobreza ha disminuido en América Latina, en casi todos los paneles de datos analizados. Con la excepción de algunos períodos, en donde se observan estancamientos en la evolución de la vulnerabilidad para algunos países, aún a pesar de que la pobreza se ha amenguado en ellos en el mismo lapso. Ello indica que no siempre la pobreza y la vulnerabilidad a la pobreza evolucionan al mismo ritmo. Pueden haber períodos de disminución de la pobreza que no van acompañados de disminuciones en la vulnerabilidad.

Se constata además que el método de estimación cumple su objetivo en forma satisfactoria. Ello valida la propuesta de Calvo (2008) respecto de la utilidad de un modelo con coeficientes específicos en este contexto, en el que la media se estima alrededor de una tendencia individual.

Una contribución relevante del capítulo, es aportar evidencia en favor de la siguiente hipótesis: a medida que los países avanzan en la disminución de la tasa de incidencia de la pobreza, disminuye la importancia relativa de la vulnerabilidad severa (determinada por la media) y aumenta la importancia relativa de la vulnerabilidad moderada (la determinada 
por el riesgo). La utilidad práctica de este hallazgo empírico es orientadora en el siguiente sentido: Aquellos países con mayores tasas de pobreza deben concentrase principalmente en atacar las causas estructurales de esta privación y preocuparse por elevar los ingresos medios de la población. Mientras que los países con menores tasas de pobreza deben orientar esfuerzos en la generación de mecanismos de seguros para disminuir el riesgo de caer en pobreza por eventos aleatorios que desvían los resultados del bienestar de los hogares bajo su retorno medio esperado.

El tercer capítulo de la tesis recibió el premio de la Academia de Ciencias Económicas de Argentina, como el mejor trabajo inédito presentado en la XLVIII Reunión Anual de la Asociación Argentina de Economía Política, celebrada del 13 al 15 de noviembre en la ciudad de Rosario, Argentina. 


\section{Contribución de la tesis a la}

\section{literatura}

Esta tesis doctoral constituye una contribución a la literatura en los siguientes aspectos:

1. Presenta un análisis crítico de más de veinte años de debate en la literatura sobre vulnerabilidad a la pobreza.

2. Indica por primera vez en la literatura que no toda la varianza es relevante para medir el riesgo de caer en pobreza, sino solamente la cola inferior de las dispersiones alrededor de la media.

3. Presenta un enfoque nuevo para identificar y medir la vulnerabilidad a la pobreza de manera coherente, utilizando solamente dos parámetros estimables: la media y la semi-desviación típica a la baja.

4. Por primera vez en la literatura se presenta un marco conceptual sobre la vulnerabilidad a la pobreza basado en un ordenamiento racional de los resultados del bienestar a partir de un modelo de media-riesgo.

5. Ofrece una útil distinción entre dos tipos de vulnerabilidad a la pobreza cuya separación es relevante para las políticas públicas: la vulnerabilidad severa determinada por la media y la vulnerabilidad moderada determinada por el riesgo asimétrico de desviarse por debajo de la media.

6. Contribuye al análisis empírico de la medición de la vulnerabilidad a la pobreza en América Latina.

7. Encuentra dos hallazgos empíricos interesantes: 
a) Existen períodos de descenso en la pobreza, acompañados de estancamientos en la disminución de la vulnerabilidad.

b) Mayores índices de pobreza efectiva, están vinculados a una mayor importancia relativa de la vulnerabilidad severa en comparación con la vulnerabilidad moderada.

De los resultados de esta tesis doctoral se derivan también nuevos desafíos para la investigación, tales como: utilizar el enfoque propuesto para medir la vulnerabilidad a la pobreza relativa y la vulnerabilidad a la pobreza multidimensional. 